\title{
Integrated World Modeling Theory (IWMT) Revisited
}

\author{
Adam Safron, $\mathrm{PhD}$ \\ Indiana University
}

\begin{abstract}
Here, I provide clarifications and discuss further issues relating to Safron (2020), "An Integrated World Modeling Theory (IWMT) of consciousness: Combining Integrated Information and Global Workspace Theories with the Free Energy Principle and Active Inference Framework; towards solving the Hard problem and characterizing agentic causation". As a synthesis of major theories of complex systems and consciousness, with IWMT we may be able to address some of the most difficult problems in the sciences. This is a claim deserving of close scrutiny and much skepticism. What would it take to solve the Hard problem? One could answer the question of how it is possible that something like subjectivity could emerge from objective brain functioning (i.e., moving from a third person to a first person ontology), but a truly satisfying account might still require solving all the "easy" and "real" problems of consciousness. In this way, IWMT does not claim to definitively solve the Hard problem, as explaining all the particular ways that things feel across all relevant aspects of experience is likely an impossible task. Nonetheless, IWMT does claim to have made major inroads into our understanding of consciousness, and here I will attempt to justify this position by discussing challenging problems and outstanding questions with respect to philosophy, (neuro)phenomenology, computational principles, practical applications, and implications for existing theories of mind and life.
\end{abstract}




\section{Table of Contents}

SOME PHILOSOPHICAL AND MECHANISTIC CONSIDERATIONS ..................................

FACING UP TO THE ENDURING PROBLEMS OF CONSCIOUSNESS WITH INTEGRATED WORLD

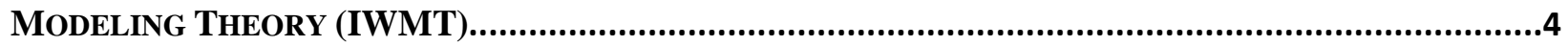

PRECONDITIONS FOR EXPERIENCE: SPACE, TIME, CAUSE, SELF, AGENCY .....................................7

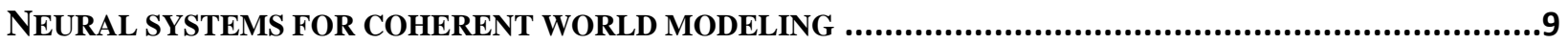

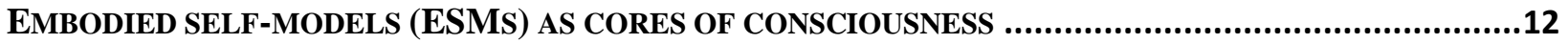

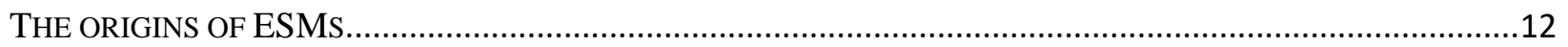

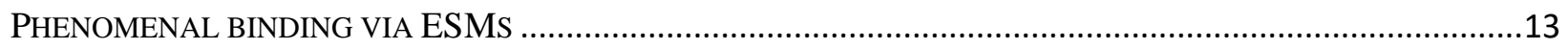

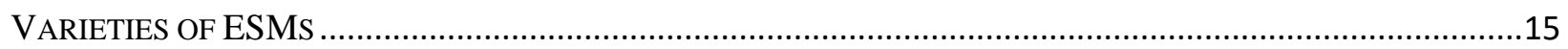

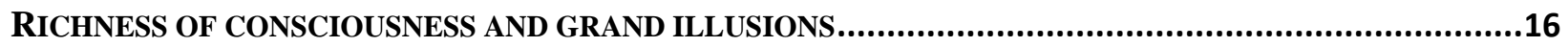

MACHINE LEARNING ARCHITECTURES AND PREDICTIVE PROCESSING MODELS OF

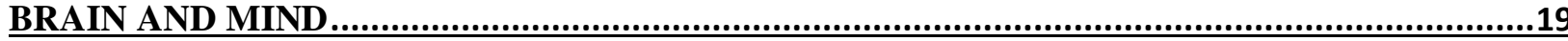

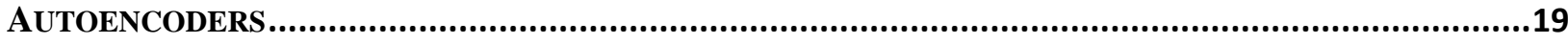

CORTEX AS FOLDED DISENTANGLED VARIATIONAL AUTOENCODER HETERARCHY.........................20

HIPPOCAMPAL COMPLEX AND HETERARCHICAL HIDDEN MARKOV MODELS; BRAINS AS

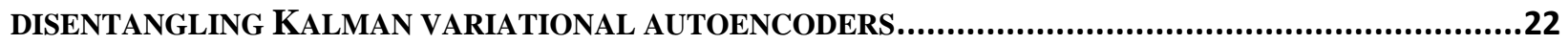

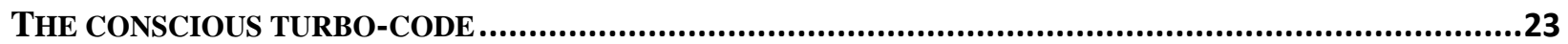

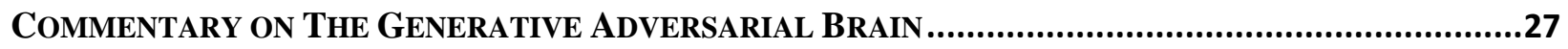

IWMT IMPLEMENTED: TOWARDS CONSCIOUS ARTIFICIAL INTELLIGENCE? ...............................29

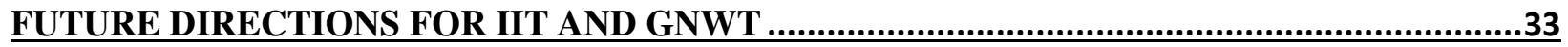

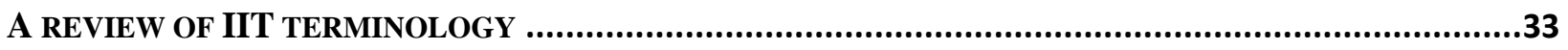

MODULES AND WORKSPACES AS COMPLEXES OF INTEGRATED INFORMATION ................................34

COGNITIVE CYCLES AND FLUCTUATING SUBSTRATES OF CONSCIOUSNESS? .................................36

MECHANISMS FOR INTEGRATION AND WORKSPACE DYNAMICS ................................................38

TOWARDS NEW METHODS OF ESTIMATING INTEGRATED INFORMATION .....................................40

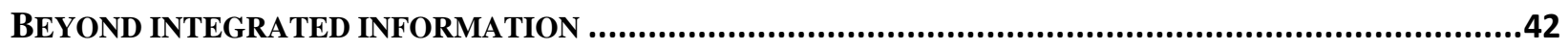

CONSCIOUS AND UNCONSCIOUS CORES AND WORKSPACES; PHYSICAL SUBSTRATES OF AGENCY ......43

BAYESIAN BLUR PROBLEMS AND SOLUTIONS; QUANTUM CONSCIOUSNESS? (NO AND YES)................45

NEUROPHENOMENOLOGY OF IWMT: QUALIA EXPLAINED? ....................................46

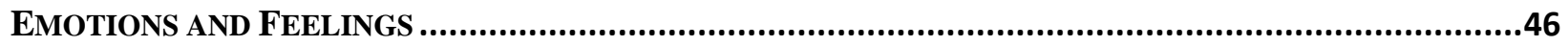

WHAT IS VALUE? PREDICTIVE CODING MODELS OF REWARD PREDICTION ERRORS .......................47

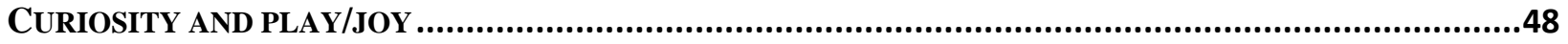

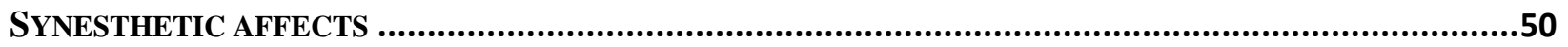

THE COMPUTATIONAL NEUROPHENOMENOLOGY OF DESIRE ......................................................51

DESIRING TO DESIRE; TRANSFORMING PAIN INTO PLEASURE AND BACK AGAIN.............................52

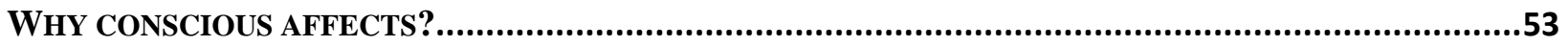




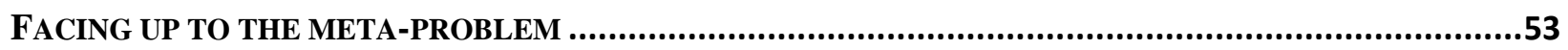

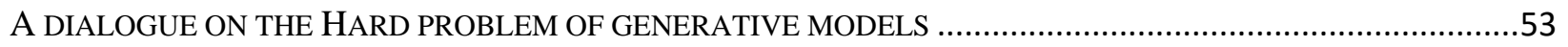

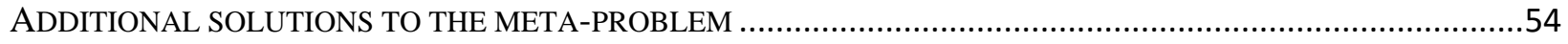

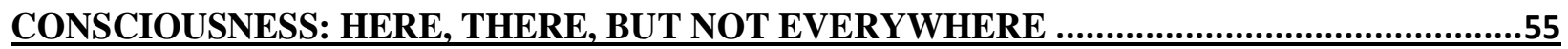

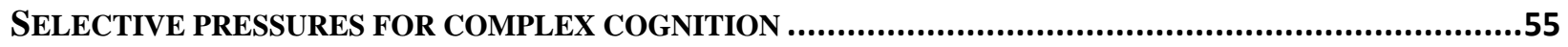

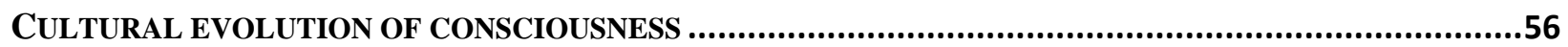

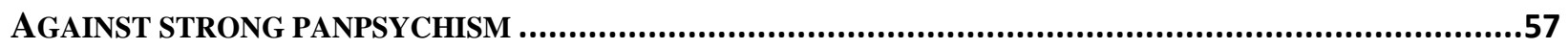

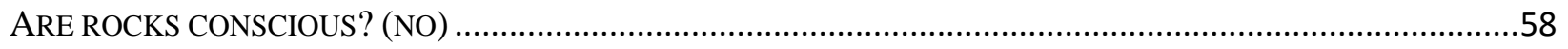

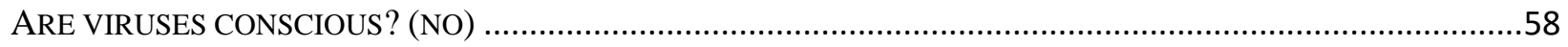

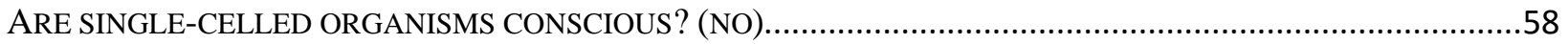

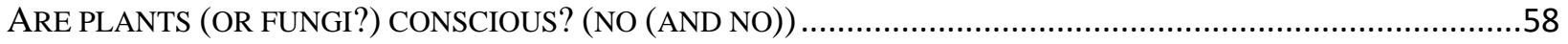

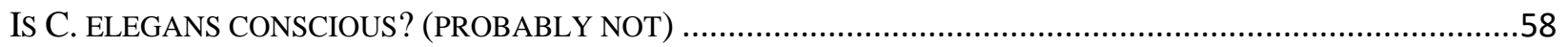

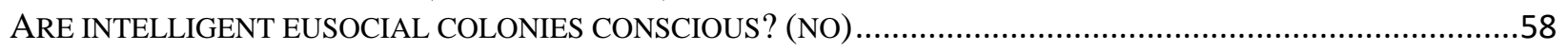

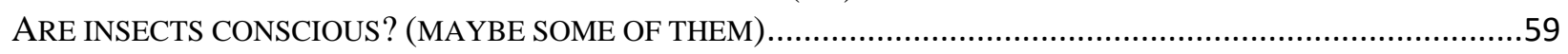

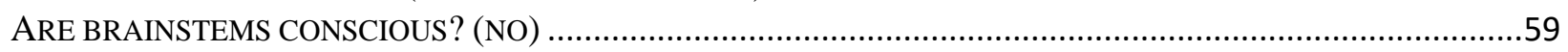

ARE REPTILES AND FISH CONSCIOUS? (MAYBE SOME (OR ALL (OR NONE)) OF THEM) .............................60

ARE OCTOPI (AND SQUIDS (AND CUTTLEFISH)) CONSCIOUS? (MAYBE (POSSIBLY (I HOPE NOT...))) ..........60

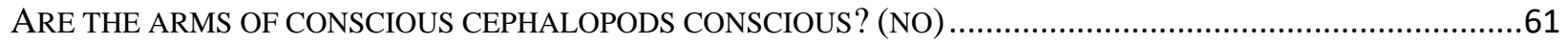

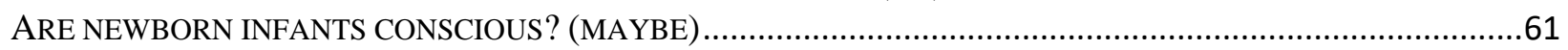

WILL ARTIFICIAL GENERAL INTELLIGENCE BE CONSCIOUS? (PROBABLY) ................................................61

A TENTATIVE TIMELINE FOR THE EVOLUTION-DEVELOPMENT OF CONSCIOUSNESS ACCORDING TO

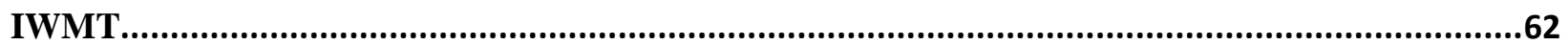

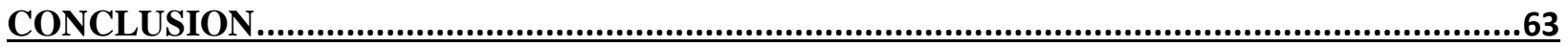

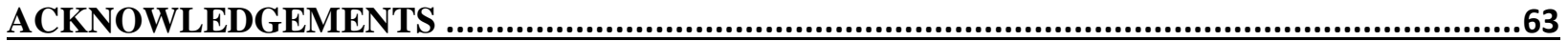

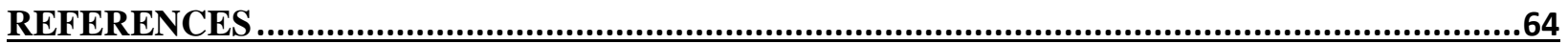




\section{Some philosophical and mechanistic considerations}

Poem

by Mary Oliver

The spirit

likes to dress up like this:

ten fingers, ten toes,

shoulders, and all the rest

at night

in the black branches, in the morning

in the blue branches

of the world.

It could float, of course, but would rather

plumb rough matter.

Airy and shapeless thing, it needs

the metaphor of the body,

lime and appetite,

the oceanic fluids;

it needs the body's world,

instinct

and imagination

and the dark hug of time,

sweetness

and tangibility,

to be understood,

to be more than pure light

that burns

where no one is--

so it enters us--

in the morning

shines from brute comfort

like a stitch of lightning;

and at night

lights up the deep and wondrous

drownings of the body

like a star.

Facing up to the enduring problems of consciousness with Integrated World Modeling Theory (IWMT)

The Hard problem of consciousness asks, how can it be that there is "something that it is like" to be a physical system (Nagel, 1974; Chalmers, 1995)? The "meta-problem" of 
consciousness refers to the (potentially more tractable) challenge of addressing why it is that opinions and intuitions vary greatly with respect to what it would take to meaningfully answer this question (Chalmers, 2018). The "real problem" of consciousness refers to the further challenges of addressing why it is that different biophysical and computational phenomena correspond to different qualities of experience (Seth, 2016).

IWMT attempts to address all these problems by combining Integrated Information Theory (IIT) and Global Neuronal Workspace Theory (GNWT) with the Free Energy Principle and Active Inference framework (FEP-AI). IIT speaks to the Hard problem by beginning from phenomenological axioms, and then goes on to postulate mechanisms that could realize such properties, ultimately coming to the conclusion that consciousness is "what physics feels like from the inside" (Koch, 2012). GNWT speaks to the real problem by focusing on the properties of computational systems that could realize the functions of consciousness as a means of globally integrating and broadcasting information from mental systems. FEP-AI has been used to address all these problems in a variety of ways, with IWMT representing one such attempt.

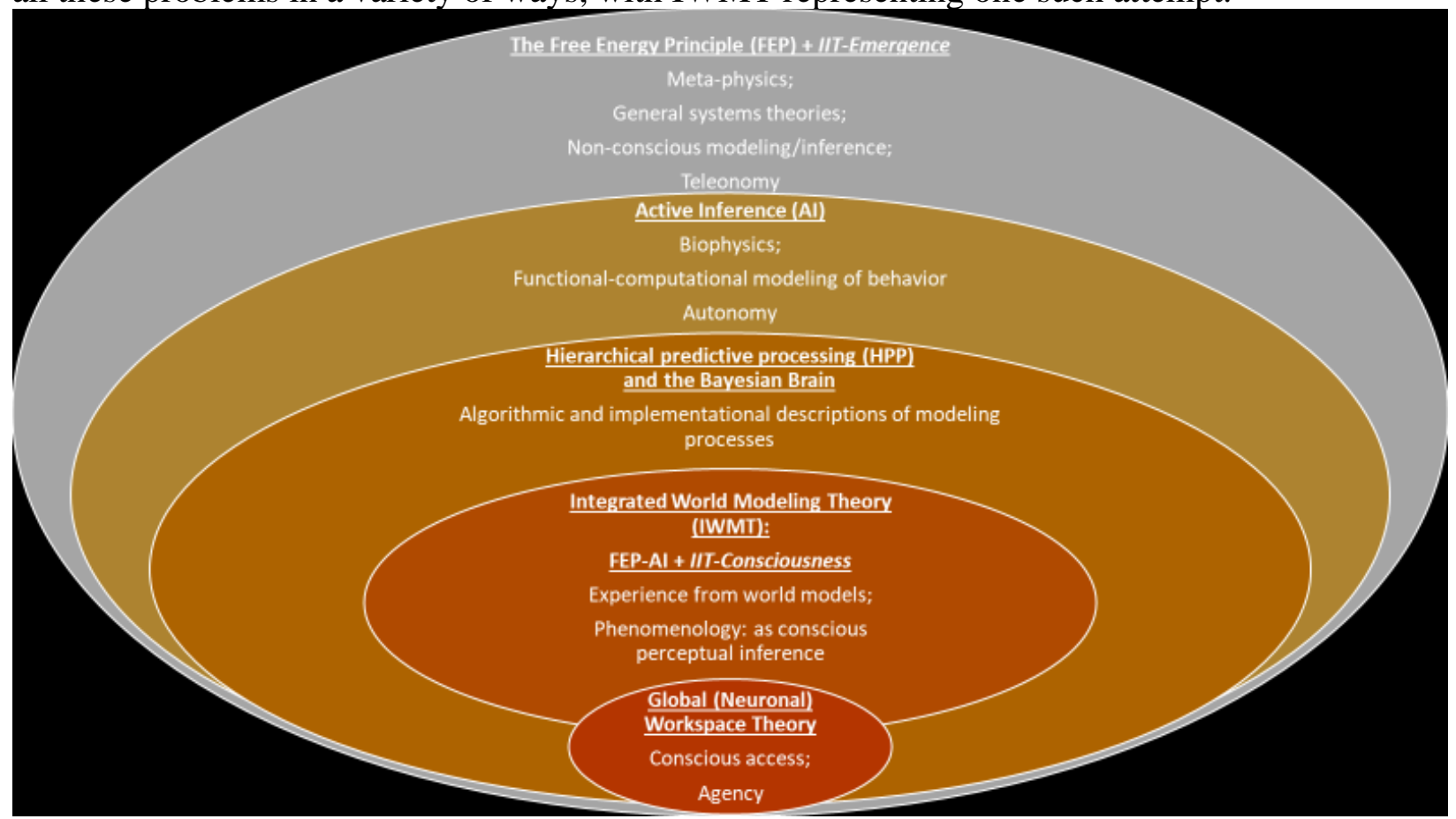

Figure 1 from "An Integrated World Modeling Theory (IWMT) of consciousness: Combining Integrated Information and Global Neuronal Workspace Theories with the Free Energy Principle and Active Inference Framework; towards solving the Hard problem and characterizing agentic causation"

The Free Energy Principle (FEP) constitutes a general means of analyzing systems based on the preconditions for their continued existence via implicit models. Integrated Information Theory (IIT) provides another general systems theory, focused on what it means for a system to exist from an intrinsic perspective. The extremely broad scope of FEP-AI and IIT suggests (and requires for the sake of conceptual consistency) substantial opportunities for their integration as models of systems and their emergent properties. Within the FEP (and potentially within the scope of IIT), a normative functional-computational account of these modeling processes is suggested in Active Inference (AI). Hierarchical predictive processing (HPP) provides an algorithmic and implementational description of means by which systems may minimize prediction error (i.e., free energy) via Bayesian model selection in accordance with FEP-AI. Particular (potentially consciousness-entailing) implementations of HPP have been suggested that involve multi-level modeling via the kinds of architectures suggested by Global Neuronal Workspace Theory (GNWT). The concentric circles depicted above are intended to express increasingly specific modeling approaches with 
increasingly restricted scopes. [Note: These nesting relations ought not be over-interpreted, as it could be argued that HPP does not require accepting the claims of FEP-AI.] This kind of generative synthesis may potentially be facilitated by developing an additional version of IIT, specifically optimized for analyzing systems without concern for their conscious status, possibly with modified axioms and postulates: IIT-Consciousness (i.e., current theory) and IIT-Emergence (e.g. alternative formulations that utilize semi-overlapping conceptual-analytic methods). Integrated World Modeling Theory (IWMT) distinguishes between phenomenal consciousness (i.e., subjective experience) and conscious access (i.e., higher-order awareness of the contents of consciousness). Non-overlap between the circle containing GNWT and the circle containing IIT-Consciousness is meant to indicate the conceivability of subjectivity-lacking systems that are nonetheless capable of realizing the functional properties of conscious access via workspace architectures. IWMT is agnostic as to whether such systems are actually realizable, either in principle or in practice.

In attempting to explain how there could be "something that it is like" to be a physical system, it is worth noting that this question is often phrased as "something that it feels like". That is, the nature of embodied perception and affective states lie at the heart of what it would take to provide a satisfying solution to the Hard problem. Further, the Hard problem could be viewed as containing an implicit begged question (that also begs to be answered): "something that it feels like, for whom?" That is, while some may want to separate consciousness from sensations or selfhood (Tononi et al., 2016), it may also be the case that addressing the Hard problem requires understanding the nature of selves, and as Dennett (2018) has argued, "free will." As will be explained in greater detail below, IWMT specifically places somatic experiences and agentic selfhood at the core of consciousness, and consciousness at the core of agency.

IWMT specifically argues that integrated information and global workspaces only entail consciousness when applied to systems capable of functioning as Bayesian belief networks and cybernetic controllers for embodied agents.(Seth, 2014c; Safron, 2019a, 2019d) That is, IWMT agrees with IIT and GNWT with respect to the integration and widespread availability of information as necessary preconditions for consciousness, but disagrees that these are sufficient enabling conditions for subjective experience. [Note: GNWT's more specific claim is that workspaces help to select particular interpretations of events, which is highly compatible with IWMT, especially with more recent Bayesian formulations of the theory (Mashour et al., 2020).] Rather, IWMT argues that consciousness is what integrated world-modeling is like, when generative processes are capable of jointly integrating information into models with coherence with respect to space (i.e., relative degrees of locality), time (i.e., relative changes within space), and cause (i.e., regularities with respect to these changes) for systems and their relationships with their environments. These coherence-making properties are stipulated to be required for situating modeled entities relative to each other with specific properties, without which there would be no means of generating an experienceable world. Generative models are stipulated to have these coherent properties because they have actual semantic content by virtue of evolving through interactions with a coherently structured (and so semi-predictable) world. IWMT further introduces a mechanism for generating complexes of integrated information and global workspaces via self-organizing harmonic modes (SOHMs), wherein synchrony both emerges from and facilitates "communication-through-coherence."(Buzsáki and Watson, 2012; Fries, 2015; Deco and Kringelbach, 2016; Atasoy et al., 2018) SOHMs are proposed to both require and allow for high degrees of meaningful integrated information, where meaning is understood as differences that make a difference to the ability of systems to pursue their goals, including the goal of modeling the world for the sake of prediction and control.

To summarize, IWMT's primary claims (as stated in the original publication) are as follows: (1) Basic phenomenal consciousness is what it is like to be the functioning of a probabilistic generative model for the sensorium of an embodied-embedded agent. (2) Higher 
order and access consciousness are made possible when this information can be integrated into a world model with spatial, temporal, and causal coherence. Here, coherence is broadly understood as sufficient consistency to enable functional closure and semiotics/sense-making. That is, for there to be the experience of a world, the things which constitute that world must be able to be situated and contrasted with other things in some kind of space, with relative changes constituting time, and with regularities of change constituting cause. These may also be preconditions for basic phenomenality (\#1), especially if consciousness (as subjectivity) requires an experiencing subject with a point of view on the world. (3) Conscious access - and possibly phenomenal consciousness - likely requires generative processes capable of counterfactual modeling with respect to selfhood and self-generated actions. Below I explore the nature of these claims in greater depth than was possible in the original publication, and also consider additional (and more speculative) considerations and future directions.

\title{
Preconditions for experience: space, time, cause, self, agency
}

\begin{abstract}
"Now what are space and time? Are they actual entities? Are they only determinations or also relations of things, but still such as would belong to them even if they were not intuited? Or are they such that they belong only to the form of intuition, and therefore to the subjective constitution of our mind, without which these predicates could not be ascribed to any things at all?... Concepts without intuitions are empty, intuitions without concepts are blind... By synthesis, in its most general sense, I understand the act of putting different representations together, and of grasping what is manifold in them in one knowledge... The mind could never think its identity in the manifoldness of its representations... if it did not have before its eyes the identity of its act, whereby it subordinates all... to a transcendental unity... This thoroughgoing synthetic unity of perceptions is the form of experience; it is nothing less than the synthetic unity of appearances in accordance with concepts."

-Immanuel Kant (1781)
\end{abstract}

\begin{abstract}
"We shall never get beyond the representation, i.e. the phenomenon. We shall therefore remain at the outside of things; we shall never be able to penetrate into their inner nature, and investigate what they are in themselves... So far I agree with Kant. But now, as the counterpoise to this truth, I have stressed that other truth that we are not merely the knowing subject, but that we ourselves are also among those realities or entities we require to know, that we ourselves are the thing-in-itself. Consequently, a way from within stands open to us as to that real inner nature of things to which we cannot penetrate from without. It is, so to speak, a subterranean passage, a secret alliance, which, as if by treachery, places us all at once in the fortress that could not be taken by attack from without."
\end{abstract}

-Arthur Schopenhauer (1844)

"Each movement we make by which we alter the appearance of objects should be thought of as an experiment designed to test whether we have understood correctly the invariant relations of the phenomena before us, that is, their existence in definite spatial relations."

-Hermann Ludwig Ferdinand von Helmholtz (1878)

By emphasizing the properties by which coherent world-modeling is made possible, the philosophical foundations of IWMT can be most strongly tied to the thought of Kant and Helmholtz. IWMT is particularly informed by Kant's stipulation of synthetic a priori categories (i.e., complex concepts possessed in advance of experience) as preconditions for judgement. IWMT further argues that these preconditions for coherent knowledge are also preconditions for coherent experience, and focuses on the categories of space (i.e., relative localization of entities), time (i.e., relative transformations of entities in space), and cause (i.e., regularity with respect to transformations). Without spatial, temporal, and causal coherence, there can be no means of situating entities relative to each other with specific properties, and so there would be no means 
of generating an experienceable world. This position is consistent with both the axioms of IIT (e.g. composition), the kind of informational synergy emphasized by GNWT, and also the constructive epistemology of FEP-AI (Swanson, 2016). IWMT goes further in emphasizing the importance of selfhood, consistent with Kant's notion of the transcendental unity of apperception in which spatiotemporal and causal information are bound together into a unified manifold via a unified experiencing subject.

Helmholtz extended Kant's project in a more empirical direction, arguing that the experience of selfhood and freedom in willing are preconditions for deriving conceptions of space, time, and cause (De Kock, 2016). According to Helmholtz, a self/world distinction and sense of agency are both required for making sense of experience, including with respect to constructing senses of space, time, and cause. This more empirically focused perspective is contrasted with Liebnizian (Sleigh, 2003) notions of "pre-established harmony" as an explanation for how minds come to be equipped with precisely the intuitions required for making sense of the world. In this way, Helmholtz rejected the a priori status of Kantian categories as part of his general project of deflating mysticism, which elsewhere involved critiquing the vitalist posit of a supernatural force to animates living things (i.e., élan vital). IWMT was developed in the same spirit as Helmholtz's naturalization of mind and nature, although with somewhat greater sympathies to notions of pre-established harmonies, since evolution by natural selection represents a means by which mental systems could come to non-mystically resonate with essential properties of the world (Ramstead et al., 2017).

Helmholtz's argument for selfhood and agency as foundational cognitive capacities is fully compatible with IWMT and FEP-AI. The centrality of agentic selfhood for sense-making is clearly expressed by Karl Friston (2017) in a discussion of "self-evidencing babies":

"What is the first and most fundamental thing a new-born infant has to do? If one subscribes to the free energy principle, the only thing it has to do is to resolve uncertainty about causes of its exteroceptive, proprioceptive and interoceptive sensations. This resolution can be achieved in one of two ways. Namely, a baby can build deep (hierarchical) models of how sensations are generated, thereby equipping those sensations with a back story or explanation that renders them predictable. Alternatively, a baby can actively sample its sensations to avoid unpredicted sensations or, more pre-emptively, to avoid situations that engender surprise... I want to focus here on structure learning and ask: what are the fundamental aspects of the model's form that enable it to provide plausible explanations for its sensory samples? It is at this point the importance of selfhood emerges - in the sense that the best explanation for the sensations of a sentient creature, immersed in an environment, must entail the distinction between self (creature) and nonself (environment). It follows that the first job of structure learning is to distinguish between the causes of sensations that can be attributed to self and those that cannot... The question posed here is whether a concept or experience of minimal selfhood rests upon selecting (i.e. learning) models that distinguish self from non-self or does it require models that accommodate a partition of agency into self, other, and everything else."

The necessity of autonomy for coherent modeling is emphasized in FEP-AI, in which expected free energy (i.e., precision-weighted cumulative prediction errors with respect to preferred states) is minimized via action/policy selection over predictive models for future (counterfactual) goal realization (Friston et al., 2017a; Friston, 2018). In these ways, IWMT supports both Kantian and Helmholtzian views on the preconditions and origins of mind.

IWMT agrees with Kant's view in that the process of bootstrapping minds (Gentner, 2010a; Tenenbaum et al., 2011; Safron, 2019d) likely requires some pre-established modes of cognitive organization (Spelke and Kinzler, 2007). For example the grid/place cells of the entorhinal/hippocampal system could contribute initial structuring of experience according to space and time (Moser et al., 2008; Buzsáki and Tingley, 2018)—although these responseproperties may substantially depend on experience for their emergence (Kropff and Treves, 
2008; Kaplan and Friston, 2018) —with a general capacity for tracking time-varying sequences being a potentially ubiquitous feature of cortex (Hawkins and Blakeslee, 2004). Implicit objective functions from innate salience mechanisms - e.g. maximizing information gain and empowerment (Redgrave et al., 2008; de Abril and Kanai, 2018)—and neuroplasticity rules such as spike-timing dependent plasticity (Hayek, 1952; Markram et al., 2011) could both be thought of as "evolutionary priors" that further help to organize experience according to likely patterns of causal influence (e.g. causes ought to precede effects). However, Helmholtz's criticism of Kant's intuitions may also highlight important differences between initial inductive biases and later constructive modeling of space (Terekhov and O'Regan, 2016), time (Buonomano, 2017; Wittmann, 2017), and cause (Buchsbaum et al., 2012). That is, it may be misleading to refer to largely innate mechanisms for structuring experience as "intuitions", as these capacities may lack experiential content by not (yet) affording sufficient coherence for the generation of an experienceable world. Finally, agency-related knowledge may be particularly complex, diverse in its forms, and dependent upon experience for its development (Kushnir et al., 2015; Kushnir, 2018; Chernyak et al., 2019).

Some open questions for future research:

- To what extent are our intuitions of space and time elaborated by our intuitions regarding causal unfoldings that depend on the agentic self as a point of view on the world (De Kock, 2016; Ismael, 2016)?

- If coherence-making is bidirectional in this way, would this imply a kind of mutual bootstrapping in learning of self, agency, and space/time/cause over the course of development?

- If sense-making involves this kind of bidirectionally, or capacity for inferential positive feedback, could the mutual dependency of subjective categories of experience partially explain non-linear shifts in psychological development (Isler et al., 2018)?

- Do different categories and intuitions asymmetrically drive different parts of development at different points in time?

\section{Neural systems for coherent world modeling}

As hopefully is made clear by the preceding discussion, philosophical considerations may be invaluable for helping to identify fundamental properties enabling conscious experience. Whether considered as synthetic a priori categories or experience-dependent constructed intuitions, the foundations of mind suggest that a primary task for cognitive science should be characterizing these properties on functional, algorithmic, and implementational levels of description. While such an analysis is beyond the scope of a single article, here I will suggest neural systems that could contribute to some of these foundational capacities.

IWMT identifies two main sources of consciousness for space: 1) a sense of locality based on body-centric coordinates (Terekhov and O'Regan, 2013), and 2) introspectable 2D maps (Haun and Tononi, 2019) organized according to quasi-Cartesian coordinates with irregular spacings biased by salience and 'navigation' potential. Body-centric spatial senses would likely primarily be found in superior and inferior parietal cortices based on convergence of the dorsal visual stream and upper levels of the somatosensory hierarchy. 2D spatial maps can be found throughout the brain, but consciously-accessible mappings are likely primarily localized to the precuneus at the brain's posterior midline. Speculatively, these precuneus-based maps may couple with the more well-known spatial maps of the entorhinal system (Moser et al., 2008), so 
allowing for 'navigating' (Kaplan and Friston, 2018) through visualized domains. That is, IWMT suggests that hippocampal representations are unlikely to themselves be directly introspectable, as deep spatiotemporal hierarchies and grounding within sensory modalities are likely required for coherent conscious experience. Precuneus-based maps may also be aligned with dynamics in the dorsomedial prefrontal cortex (another midline structure) (Hassabis et al., 2014; Li et al., 2018; Faul et al., 2020), the latter of which is here interpreted as sources of "attention schemas" (Graziano, 2019), upper levels of action hierarchies, and-perhaps most saliently with respect to conscious awareness - as an additional level of hierarchical control over the pre-supplementary eye fields. With precise sequencing shaped by striatal-thalamic-cerebellar loops (Gao et al., 2018), these frontal representations may provide a source of coherent vectors for directing the "mind's eye", so influencing what is likely to be 'projected' onto the precuneus as a kind of inner 'theater.' Mechanistically, these action-oriented influences on perception may further depend on pulvinar-mediated synchrony for their realization (O'Reilly et al., 2017; Hu et al., 2019).

IWMT suggests that we ought to expect all phenomenal content to involve spatial aspects, potentially requiring multi-level processes of spatialization. Recent evidence suggests that we parse complex features by performing a kind of multidimensional scaling (Hout et al., 2013 ) in which features are mapped onto $2 \mathrm{D}$ spaces. The entorhinal/hippocampal system may be particularly important for establishing these mappings (Bellmund et al., 2016, 2018; Nau et al., 2018), and potentially for establishing the routes by which we are able to make sense of these complex domains by performing (generalized) 'navigation' through their spatialized representations. For example, it has recently been demonstrated that entorhinal grid cells are used to spatially organize reward-related representations in the ventromedial prefrontal cortex (another midline structure), with spatialization of task structure having behavioral significance for reinforcement learning problems (Baram et al., 2019).

The nature of time perception may be somewhat more complicated compared to space, and may even be conceptually derived from initially spatial understanding (Levin et al., 1978; Levin, 1992). While the entire brain (or at least much of the neocortex) may be sensitive to temporally varying sequences (Hawkins and Blakeslee, 2004), there seems to be no singular clock for time perception. One candidate clock-like mechanism potentially influencing conscious time perception includes the formation of "global emotional moments" via the insular salience hierarchy (Craig, 2009b), with a greater density of salient events corresponding to relatively slower experienced (but not necessarily remembered) temporal unfolding. Speculatively, dopaminergic influences on time perception (Soares et al., 2016; Buonomano, 2017) potentially suggests that the ability to both track and simulate (and track via simulations) causal sequences via actions may provide another factor influencing time perception, with a greater frequency of actions corresponding to elongated subjective timelines. Non mutually exclusively, relationships between dopamine and time consciousness could be hippocampally mediated (Mannella et al., 2013; McNamara and Dupret, 2017). These hippocampal influences could include multiple factors, such as the frequency with which events are encoded as new memories, or through the mapping of timelines onto (2D) spatial trajectories with grid/place cells. Indeed, the ability of the entorhinal system to construct maps and routes for navigation (broadly construed) may be a primary means by which space and time come together in brain and mind. Such simultaneous localization and mapping mechanisms may provide a basis for both the spatialization of time as well as the temporalization of space, as these two modes of quantization are fundamentally linked (and mutually defined) in terms of velocity, which may be physiologically linked via locomotion-dependent cholinergic midbrain nuclei (Lee et al., 2014). That is, velocity estimation 
both requires and enables the ability to track changing relative spatial locations, with speed being time-varying displacement within space. Speculatively, similar relationships between time and space might also be mediated by mapping events onto body maps, both in terms of using bodily representations as a kind of space (within which things can change at varying speeds), as well as via potential magnitude estimation via the intensity of proprioceptive and interoceptive sensations. Finally, for linguistic beings such as humans, it may be difficult to overstate the importance of analogical/metaphorical construction processes for tying together and expanding these fundamental categories (Jaynes, 1976; Lakoff and Johnson, 1999; Safron, 2019a).

Causal understandings may be more difficult to map unto neural systems than time and space. As previously mentioned, some proto-causal understanding may derive from mechanisms such as the ability of spike-timing dependent plasticity to arrange events into likely time-varying sequences (Hayek, 1952; Markram et al., 2011) —wherein causes can be expected to precede events — or via salience mechanisms such as modulation of midbrain dopamine by whether events are likely to have been internally or externally generated (Redgrave et al., 2008; de Abril and Kanai, 2018). However, understanding causation requires more than these proto-intuitions, and in particular the ability to generate counterfactual scenarios involving simulated interventions, potentially providing an implementation of the "do operator" introduced by Judea Pearl for causal inference with graphical models (Pearl and Mackenzie, 2018). That is, while it is unclear whether anything like the graphical representations underlying Pearlean analysis are used by brains and minds, the ability to simulate a variety of actions/interventions could provide a basis for the same kinds of causal reasoning. However, as will be discussed in greater detail below, this ability to generate counterfactual scenarios likely required the advent of internal dynamics that can be decoupled from immediate engagement with the environment. Intriguingly, such adaptations may have arisen relatively straightforwardly with increasing degrees of cortical expansion, some of which may have provided a difference in kind with respect to expanded association cortices and a more freely operating default mode network (Buckner and Krienen, 2013; Sormaz et al., 2018).

Finally, while the potential complexities of selfhood are inexhaustible, a very minimal sense of self and agency could potentially be derived from the reliable ability of embodied brains to learn that bodies depend on particular sensors by which they can perceive and effectors by which they can act. Since sensors and effectors are located on and in the body-and not elsewhere- the fact that bodily states are uniquely perceivable and controllable may provide a relatively straightforward means of construing models in which an agentic self exists as a separate entity from the rest of the (less immediately perceivable/controllable) world. While a broad range of neural systems may contribute to self-consciousness in diverse ways, below we will focus on the potential importance of body maps for scaffolding inferences about selves and the (life)worlds in which they find themselves embedded.

Some open questions for future research:

- What are the sources of a 3D sense of space? Is visual consciousness always ultimately experienced as a 2D projection, with depth provided by differences between generated points of view (changing perspective either overtly or covertly in imagination), and a 3D world corresponding to richer transition models?

- In what ways do the nature of spatial representations vary in birds and flying mammals (Sarel et al., 2017)?

- In what ways do 2D maps and body-centric spatial senses interact? 
- What are the functional significances of integrative 2D maps found at cortical midline structures, and in what ways do these interact with (also medially located) cingulate cortices?

- How are egocentric and allocentric perspectival reference frames combined and switched between within an individual's mind?

- What are the range of mechanisms contributing to different kinds of time perception?

\section{Embodied self-models (ESMs) as cores of consciousness}

(For additional discussion of the potential developmental and functional significances of ESMs, please see Safron (2019d): "The radically embodied cybernetic Bayesian brain; towards explaining the emergence of agency.")

The origins of ESMs

IWMT argues that ESMs form foundational cores and scaffolding for numerous and varied mental processes, ranging from the handling of under-constrained inference spaces to the intentional control of attention, imagination, and action. ESMs are both body maps and cybernetic control hierarchies, constituted by action-perception cycles grounded in skeletal muscle and associated perceptual efferents. IWMT further proposes that the centrality of ESMs is to be expected based on early embodied experiences (Smith et al., 2018b; Whitehead et al., 2018), which may serve as learning curriculums in which conditions for inference are fruitfully constrained (Pfeifer and Bongard, 2006), so allowing organisms to bootstrap their ways toward handling increasingly challenging hypothesis spaces within zones of proximal development (Vygotsky, 1934). With respect to the challenge of constructing robust causal world modelsboth enabling and possibly entailing conscious experiences - the combinatorics of unconstrained inference spaces may only be surmountable via the inductive biases afforded by embodied learning. This fundamentally somatic developmental legacy suggests a radical perspective in which ESMs form a semi-centralized scaffolding for all intentional (and many unintentional) mental processes, grounding abstract symbolic thought in mental simulation and metaphorical extension from initial embodied experiences (Lakoff and Johnson, 1999; Lakoff, 2014).

ESMs may provide means by which action selection can be influenced via iterated comparisons of sensed and imagined sensorimotor states, with much complex planning achieved through backward chaining from goals, implemented via predictive coding mechanisms. In this view, intentions are proposed to function as systemic causes with respect to neural dynamics, arising through interactions between beliefs and desires (as counterfactual predominantlyinteroceptive beliefs). Additionally, neuronal ensembles underlying ESMs may be positioned as mid-level (in terms of hierarchical abstraction), centrally located richly connected nodes in generative neural networks. The coherent organization of these ESM nodes (because of connections to shared embodied causes), may contribute to the overall effective small-world properties of brains, so greatly enhancing message-passing potential, so greatly enhancing capacity for informational integration. In this way, in addition to being at the core of most mental processes, ESMs may tend to be at the center of dynamic cores (Edelman et al., 2011a; Safron, 2019d) as complexes with high degrees of integrated information, so instantiating communication backbones for global workspaces. With respect to this hypothesis of workspace dynamics via ESMs, it is notable that periods of high and low modularity have been observed to 
most strongly vary based on degrees of activity within sensorimotor and visual networks (Betzel et al., 2016), potentially suggesting pivotal roles for these systems in integrating information across otherwise isolated modules. In addition to constituting the most extensive resting-state component involving $27 \%$ of overall grey matter (Tomasi and Volkow, 2011), sensorimotor networks have recently been identified as helping to establish a core of functional connectivity (Battiston Federico et al., 2018), with high degrees of overlap and coupled activity with other functional networks, including the default mode and its contributions to establishing global workspaces and the foundations of selfhood (Davey and Harrison, 2018; Deco et al., 2019; Wens et al., 2019).

\section{Phenomenal binding via ESMs}

High degrees of mutual information across ESMs may enable high capacities for selforganized synchrony and inferential stability (Marshall and Meltzoff, 2015; Whitehead et al., 2018). Indeed, the ability of body-centered neural responses to provide a source of more general stability may be expected by their early emergence with respect to both ontogeny and phylogeny. Along these lines, recent work investigating the development of functional networks in the spines of zebra fish have demonstrated that motor-neurons begin a stereotyped process of enabling global synchronization dynamics, beginning with the reliable establishment of increasing degrees of synchronous local activity (Smith et al., 2018a), followed by larger scale integration (or self-organization) into well-defined oscillatory modes as critical thresholds are surpassed (Wan et al., 2019). Further, high degrees of integrative capacity among body maps may potentially help to explain the remarkable capacities of nervous systems to reconfigure themselves for both good (e.g. recovery after injury) and ill (e.g. phantom limb syndrome) (Ramachandran et al., 1999; Blakeslee and Blakeslee, 2008; Valyear et al., 2020).

Based on these considerations, IWMT proposes that ESMs may transfer some of their synchronous (and inferential) stability to non-body representations (e.g. external objects). That is, synchrony for non-body representations may be enhanced by functionally coupling with the synchronous dynamics of ESMs. This coupling could potentially be realized by the driving of simulated (and sometimes enacted) actions by reactive dispositions and perceived affordances (Gibson, 1977). Specifically, affordance relations must have some kind of physical basis in neuronal ensembles - even if highly dynamic and context sensitive - constituted by representations of action-perception cycles, grounded in the effectors and sensors of the body. If non-body representations are auto-associatively linked to ESMs via affordance representations, then synchronous dynamics within ESMs could transitively entrain the synchronous dynamics of non-body representations, so increasing their perceptual stability. With relation to perceptual binding, specific affordances could contribute to specific patterns of synchrony, so instantiating specific networks of integration, which in some instances may entail phenomenal (and access) consciousness.

Recent work from the lab of Michael Graziano (Guterstam et al., 2019) may provide support for this model of perceptual binding via ESM-based affordances. In Attention Schema Theory (AST) (Graziano, 2013, 2019), conscious awareness is thought to correspond to reduceddimensionality schematic models of attention, which provides an informational object that is simpler to predict and control, relative to that which is modeled. The sketch-like nature of attention schemas makes them not amenable for clear introspection, so contributing to an anomalous inference wherein awareness is implicitly (and sometimes explicitly) viewed as a 
fluid-like physical substance that comes out of a person's eyes and reaches out into the world, so contributing to the "extramission myth of visual perception." In a recent study, Graziano and colleagues (2019) found that people's estimates of where an unstable object might fall were influenced by seeing others have an available line of sight. That is, someone else's gaze seemed to result in an inference of force transfer in the direction of the unstable object. This finding is consistent with the ESM-based model of perceptual binding described above, although variations on the experiment might provide an opportunity to uniquely test the hypotheses proposed here. According to the "eye beams" model of AST, the implicit force associated with gaze should always be a push — due to the anomalous inference that awareness is a fluid that can be emittedcausing the object to be more likely to fall away from observers. However, according to the model of phenomenal binding via ESMs, the force may be either a push or a pull, depending on associated affordances, and possibly affective states.

Mechanistically, traveling waves (Muller et al., 2018; Zhang et al., 2018; Roberts et al., 2019) from ESMs may form major points of nucleation for the formation of self-organizing harmonic modes (SOHMs) (Safron, 2020) as large-scale meta-stable rhythmic attractors (Deco and Kringelbach, 2016; Atasoy et al., 2018, 2019; Deco et al., 2019; Lord et al., 2019), so being able to influence the global coordination of brain activity. This model of resonant binding via simulated embodied engagements suggests patterns of neuronal synchronization could potentially be linked to either the overt or covert specific actions. As described in Safron (2019d), partially-expressed motor commands may provide a basis for not just attentional control, but also the intentional driving of working memory and action (Schurger et al., 2012; Fifel, 2018; Imaizumi and Tanno, 2019; Suzuki et al., 2019). Thus, ESMs may form cores of consciousness as dominant sources of integrated effective connectivity across the entire brain, both with respect to coherent perception and action. This model of ESM-grounded consciousness would not only imbue all percepts with the affordance potential of sensorimotor contingencies (O'Regan and Noë, 2001), but also the sense of "presence" as perceptual depth from counterfactual richness (Seth et al., 2011b; Seth, 2014a), so helping to explain potentially fundamental aspects of phenomenology.

This radically embodied view has received some support from recent advances in wholebrain calcium imaging, which reveal that motor information dominates nearly every aspect of neural signaling in almost every modality (Drew et al., 2019; Musall et al., 2019). Further support for this model can potentially be found in the finding that parietal cortex is a source of both high-level body representations as well as spatial awareness. Notably, parietal damage can not only result in anosagnosia and alien limb syndromes, but also hemi-spatial neglect (Graziano, 2018b). Along these lines, there is the counter-intuitive finding in which the spatial extent of neglect symptoms are extended via providing a reach-extending tool (Cardinali et al., 2009) for the hand corresponding to the affected side of space (Graziano, 2018a). The model of affordance-based bindings via ESMs described above may potentially provide a partial explanation for this surprising empirical phenomenon. Specifically, if neglect symptoms are due to disrupted binding, then the extension of body maps into tools could have the consequence of extending the scope of pathology, due to failed integration via coupling with ESMs whose normally coherent dynamics have been compromised. Indeed, resonant coupling with ESMs may be one of the few models capable of explaining the mechanisms whereby external objects (potentially including other agents) may become incorporated into body maps (Miller et al., 2019), with synchronous motions helping to establish this expansion/binding. Further support for potentially radically-embodied bases for phenomenality may also be found in the findings of 
impaired memory associated with induced out-of-body states (Bergouignan et al., 2014), and superior memory associated with $1^{\text {st }}$ person points of view (St. Jacques, 2019).

\section{Varieties of ESMs}

In these ways, ESMs would function both as sources of maximal model evidence in FEPAI, complexes of maximal integrated information in IIT, and global workspaces in GNWT. This account of consciousness and agency centered on ESMs is consistent with both the Informational Closure (Chang et al., 2019) and Intermediate Level (Prinz, 2017) theories of consciousness. Specifically, intermediate levels of abstraction allow for embodied simulation (Varela et al., 1992; Barsalou, 2009, 2010), where action-perception cycles allow for cybernetic sense-making and grounded cognition. Indeed, IWMT's focus on cybernetic grounding (via ESMs) could help to explain why consciousness may arise "only at the personal level" (Elton, 2000).

ESMs are likely composed of multi-layer control hierarchies at varying levels of abstraction, ranging from $1^{\text {st }}$-person interfaces-i.e., the "lived body" (Tani, 2016; Rudrauf et al., 2017) - to $3^{\text {rd }}$-person body schemas capable of acting as symbolic and self-reflexive intentional controllers. That is, the singular embodied self and models of selfhood as a "center of narrative gravity" (Dennett, 2014; Davey and Harrison, 2018) imply multiple roles for unified embodied representations as high-level controllers, organized according to multiple perspectival reference frames. Crucially, the complexity and specificity of models of self and world can be greatly expanded by the combinatorial and recursive properties of language (Damasio, 2000; LeDoux, 2019), including temporal extension and stabilization via organization into diachronic narratives. In these ways, while consciousness may not depend on language for its realization, linguistic capacities may have profound impacts on the evolution and development of consciousness.

Thus, multi-level integration via selfhood may represent a necessary condition for perceptual coherence by providing a source of binding from core embodiment. Further, in line with renormalization group theory and the enslaving principle of synergetics (Haken, 1977; Butterfield, 2014), the ability of self-processes to stably persist through time may provide reduced-dimensionality attracting center manifolds capable of bringing order to — or generating selective pressures over-faster dynamics at lower levels of organization. That is, a slower, larger, and more centrally positioned set of dynamics has an asymmetric potential to entrain (or enslave) faster and more fleeting processes, which will be relatively less likely to produce coherent flows of influence due to their transient character. In these ways, self-processes can be viewed as sources of highly coherent meso- and macro-scale vectors — or effective field theories — over biophysical dynamics, so allowing systems to explore regions of state space in ways that would otherwise be unlikely to occur without centralized integrative structures.

Selves may provide spatial and temporal structure for complex sequences at multiple levels of abstraction, including symbolically. Such abstract integrative structures are referred to as "narratives" (Hirsh et al., 2013), for which it is no coincidence that such modes of organization may allow for optimal learning, and for which it also may be no coincidence that the act of telling and listening to stories is a human universal (Harari, 2015; Henrich, 2017). In terms of control systems, narratives allow for coherent stabilization of evolving conceptual structures in ways that provide multi-level syntax, so affording planning on multiple temporal and spatial scales. Narratives with multi-scale organization may provide one of the best ways to model and control such extended processes, including with respect to the narrative processes that 
both help to generate and are governed by self-models. That is, agentic selfhood can be viewed as a story that becomes (more or less) true with the telling/doing/enacting.

In brief, at their most basic, selves are constituted by models of embodiment and embedding within the external environment. At their most complex (Fujita et al., 2018), selves may be viewed as patterns with which an agent-like-system most consistently endorses and identifies with over time, where the agentic system is construed according to a kind of projected revisionist victor's history (Dennett, 2014, 2017; Mahr and Csibra, 2017), where victors are constituted by dominating coalitions of patterns, largely bound together by evolving interactions between habits, narratives, and the specific niches constructed by agents. Inter-temporally coherent belief-desire coalitions are more likely to consistently achieve higher value (Ainslie, 2005; Lewis, 2015), and so will tend to be reinforced, and so will tend to dominate subsequent persona evolution. The inter-individual shared narratives co-evolving with these pattern coalitions (Peterson, 1999; Harari, 2015; Shiller, 2019) will be shaped by repeated games both within (Edelman, 1987; Minsky, 1988; Ainslie, 2010) and between individuals (Traulsen and Nowak, 2006; Tomasello, 2014). Although self-processes may become extremely complex in these kinds of ways, in all cases they both originate from and must continually deal with the constraints and affordances of their fundamentally embodied nature.

\title{
Richness of consciousness and grand illusions
}

\begin{abstract}
"For my part, when I enter most intimately into what I call myself, I always stumble on some particular perception or other, of heat or cold, light or shade, love or hatred, pain or pleasure. I never can catch myself at any time without a perception, and never can observe any thing but the perception. When my perceptions are remov'd for any time, as by sound sleep; so long am I insensible of myself, and may truly be said not to exist. And were all my perceptions remov'd by death, and cou'd I neither think, nor feel, nor see, nor love, nor hate after the dissolution of my body, I shou'd be entirely annihilated, nor do I conceive what is farther requisite to make me a perfect non-entity. If any one upon serious and unprejudic'd reflexion, thinks he has a different notion of himself, I must confess I can reason no longer with him. All I can allow him is, that he may be in the right as well as I, and that we are essentially different in this particular. He may, perhaps, perceive something simple and continu'd, which he calls himself; tho' I am certain there is no such principle in me... But setting aside some metaphysicians of this kind, I may venture to affirm of the rest of mankind, that they are nothing but a bundle or collection of different perceptions, which succeed each other with an inconceivable rapidity, and are in a perpetual flux and movement." -David Hume (1739)
\end{abstract}

According to IWMT, not only is there something of a Cartesian theater, but things may be re-presented on/in this VR screen. And as described elsewhere (Safron, 2019d), quasihomunculi (multi-modal action-oriented body maps) may introspect the contents of these spaces with partially-expressed motor commands, with simulated saccades potentially being particularly important as both a) a source of the spotlight of attention, and b) coherent vectors for intention and action. However, what is the extent of this unified field of experience? Do we actually fill in a full and rich simulated environment, or is this subjective experience some kind of "grand illusion", where in fact we are only filling-in local aspects of the environment in an ad hoc fashion (Dennett, 1992, 2018; Noë, 2002; Abid, 2019)? Rather, we might not actually fill in a more complete sensorium all at once, but instead may fill it in a way that would reflect the sensory acuity accompanying our actual sensorimotor engagements, which may be surprisingly limited (e.g. proportional to the narrow field of view afforded by the focal region of the retina). Compelling support for a surprisingly low-dimensional consciousness is described in Nick Chater's (2018) book, “The Mind is Flat.” Reviewing evidence including change blindness 
and the limitations of feature-based attention, Chater argues that much of experience may be considered to be on a spectrum with neuropsychological conditions such as simultanagnosia, wherein patients are unable to perceive more than one item at a time. Referring to the limits of feature perception, Chater writes: "It is as if the visual system has a single metaphorical hand that can reach out, select, and manipulate just one pattern at any given moment." As described above with respect to ESM-based phenomenal binding, IWMT considers this the suggestion of hand-based attentional selection to be something more than a 'mere metaphor.' Rather, the necessity of grounding cognition in sensorimotor states may substantially contribute to both the capacities and constraints of conscious processing.

Chater further describes both intelligence and mental categorization in terms of analogical reasoning and a process of similarity-based matching via "perception-memory" resonance. This view of intelligence as grounded in analogy-making is consistent with views in which analogical reasoning is a core cognitive process (Hawkins and Blakeslee, 2004; Shanahan and Baars, 2005; Gentner, 2010a; Hofstadter and Sander, 2013; Lakoff, 2014; Safron, 2019a). The proposed mechanism of resonant matching is also consistent with Edelman's description of perception as the "remembered present" (2001) and Grossberg's (2017) account of memoryperception via recurrent neural dynamics. Analogical reasoning also plays a central role in IWMT, in that much of the stream of consciousness may evolve through a process of iterated comparisons between (potentially low-dimensional) quale states (Safron, 2019d).

Phenomena such as the invisibility of the ocular blind spot suggests that some kind of perceptual filling occurs - and which is something generative models are well-suited to provide. However, the extent of this pattern completion remains unclear, and may be surprisingly sparse. For example, to what extent does the "visuospatial sketchpad" model of working memory actually involve a kind of internal sketching, potentially even involving the internalization of actual experiences with drawing (Tversky, 2019)? Some supporting evidence for lowdimensional inner-sketching can be found in recent work in which the top-down generation of internal visual models is well-described by line drawings (Morgan et al., 2019). The authors note that such images can be traced back to Paleolithic hunters 40,000 years ago (with possibly earlier origins), suggesting that line drawings may represent both effective means of conveying meanings, and may also reveal functional principles of the visual system. While this particular study focused on predicting responses in the ventral stream, it may also be the case that patterns of neural activity in the precuneus have semi-topographic correspondences with the contents of visual consciousness. That is, feature hierarchies of the ventral stream may help to coordinate evolving spatiotemporal manifolds in the precuneus as a consciously accessible 2D sketchpad. Connections with upper levels of various sensory hierarchies would further allow for the (partial) filling-in of multi-modal somatosensory states, providing a basis for not just a Cartesian theater, but fully immersive virtual reality (Metzinger, 2010). Even more, connections between these various modalities of experience with the entorhinal/hippocampal system could allow this somatic pattern-completion to evolve according to trajectories through physical and abstract spaces, so providing a basis for episodic memory and novel imaginings. With respect to the filling-in process, the actual contents of consciousness would likely depend on the specific degree to which representations from various sensory hierarchies are capable of coupling with large-scale meta-stable synchronous complexes (i.e., self-organizing harmonic modes, or SOHMs) on the timescales at which they form.

While conscious experience may be "flat" (Chater, 2018), in terms of being of surprisingly low dimensionality, IWMT argues that the functioning of consciousness within an 
overall mental system may also be deep. This point about the multiply-determined contextual significances of reduced-dimensional processing may be well-expressed in terms of recent findings that nearly all languages may converge on a common information transmission rate of $\sim 39$ bits/second (Coupé et al., 2019). Indeed, the low dimensionality of conscious processing may be the primary reason for this communicative bottleneck. However, the generative potential of consciousness and expressive power of language (with its "infinite use of finite means") may nonetheless be supra-astronomical with respect to their semiotic capacities. That is, even if integrative dynamic cores and global workspaces have extremely limited capacities, they may nonetheless possess depth and powerful combinatorics via spanning levels both within and across hierarchically-organized systems, so constituting a multi-scale functional heterarchy. Additionally, the temporally-extended nature of conscious processes (Buonomano, 2017; Wittmann, 2017) affords numerous and varied opportunities for shaping by a rich collections of unconscious dynamics, many of which might be given coherent organization by a diverse-but capable of being integrated, to varying degrees - assortment of self- and world- modeling processes on multiple levels (and their linguistic elaboration).

In these ways, while some of the richness of consciousness may represent a "grand illusion", in many ways this supposedly illusory phenomenon may function as if a rich and full field were always present by filling in details on an as-needed basis. That is, in addition to having many of the "varieties of free will worth wanting" (Dennett, 2003), we may have many of the varieties of conscious experience worth wanting as well. Consciousness would only appear to be "flat" if we consider it on a momentary basis, rather than as a temporally extended unfolding of a generative process, which IWMT considers to be a more accurate point of view. In this way, the illusory nature of rich consciousness may itself be something of an illusion due to trying to model inherently temporally-extended processes with non-temporal reference frames, which would be prima facie inadequate for evaluating the relevant phenomena. (Note: Deflations of deflationary accounts of selfhood can be arrived at in a similar fashion, including with respect to Buddhistic/Humean reductions of selfhood to non-self elements.)

Some open questions for future research:

- To what extent does experiential filling in proceed as a series of discrete "snapshots" (Crick and Koch, 2003; Madl et al., 2011; Herzog et al., 2016), or as a continuous flow for the "stream of consciousness"?

- To what extent is there a semi-topographic mapping between (temporally-extended) events in the world and traveling waves in the brain?

- To what extent do individuals who report a lack of mental imagery (i.e., aphantasia) have atypical neural and experiential dynamics (Zeman et al., 2015; Keogh and Pearson, 2018; Wicken et al., 2019), and do some aphantasic individuals have relatively typical levels of visualization abilities, but with reports of a lack of imagery involving a different (and potentially more accurate) interpretation of typical consciousness?

- To the extent that aphantasic individuals have atypically configured minds and brains, could this help identify necessary neural systems for different aspects of visual awareness (E.g. precuneus as Cartesian theater, posterior cingulate as "mind's eye", and dorsomedial prefrontal cortex as source of attentional vectors)? 


\section{Machine learning architectures and predictive processing models of brain and mind}

We have reviewed some of the philosophical issues and neural systems relevant to integrated world modeling. We will now expand these analyses to show how many of these processes and underlying systems may also be describable in terms of computational principles from machine learning. It may seem rather implausible that present technologies could reveal deep principles about the nature of mind, with potentially cautionary tales to be found in previous metaphorizations based on the technology of the day. Is this just another case of naïve arrogance of overgeneralizing from the familiar and fashionable, akin to previous claims that minds could be understood in terms of the accumulation and release of pressures, or when nervous systems were suggested to function according to the logical operations found in computers (McCulloch and Pitts, 1943)? Metaphors in which brains are understood as computers and even steam engines are both consistent with IWMT and the Free Energy Principle and Active Inference (FEP-AI) framework. That is, not only is there necessarily a sense in which brains compute information, but where the serial operation of conscious access may even be thought of as a kind of (neural) Turing machine (Dehaene, 2014; Graves et al., 2014). Even more, if neural systems minimize (variational) free energy, then this not only provides computational justification for pressure-based analogies (Carhart-Harris and Friston, 2010), but potentially even analogies drawing on the causal powers of engines as systems that perform thermodynamic work cycles (Safron, 2019d, 2020). Thus, these previous attempts to analogize the nature of mind with existing technologies may have been surprisingly prescient.

Considering that FEP-AI can trace its origins back to Helmholtz machines and autoencoders (Dayan et al., 1995), the rise of deep learning may have afforded conceptual progress for understanding not just minds, but all dynamical systems (viewed as generative models). The idea that deep learning could potentially inform neuroscience ought to be relatively unsurprising (Hassabis et al., 2017; Richards et al., 2019), in that artificial neural networks were designed to try to capture relevant aspects of nervous systems, albeit with limited physiological detail and some biologically implausible functionalities (e.g. training by backpropagation). IWMT goes further in arguing that not only can useful computational principles be derived from machine learning, but some architectures may have close correspondences with the neural processes contributing to consciousness via coherent world modeling. Below I will review a few of these relevant technologies and the ways functionally equivalent processes might be realized in biological systems.

\section{Autoencoders}

Autoencoders use encoder networks to compress higher-dimensionality data into lowerdimensionality feature representations (Kramer, 1991), which are then used by generative decoders to reconstruct likely patterns of higher-dimensional data. These generative models are typically trained by minimizing the reconstruction loss between initial input layers of encoders and output layers of generative decoders. Autoencoders allow richer feature descriptions to be generated from highly compressed or noisy inputs, and can also be used to infer missing data (e.g. filling pixels for occluded sensors).

Variational autoencoders separate the low-dimensionality output of encoders into separate vectors for means and variances, from which sample vectors are derived as inputs to 
generative decoders, so allowing data to be generated with novel feature combinations (Kingma and Welling, 2014; Doersch, 2016). Training proceeds by simultaneously minimizing both the reconstruction loss as in non-variational autoencoders, as well as the KL-divergence between the posterior distributions and priors - often a unit Gaussian — so preventing overfitting of observed data and inducing more evenly distributed models of latent feature spaces with more interpretable features. Disentangled variational autoencoders have a precision-weighting term applied to the KL-divergence - isomorphic with Kalman-gain in Bayesian filtering - increasing or decreasing the degree to which different (combinations of) feature dimensions are weighted, so allowing more reliable/useful information to be more heavily leveraged during training and inference.

\section{Cortex as folded disentangled variational autoencoder heterarchy}

A predictive coding model of cortex may be approximated by folding a disentangled variational autoencoder over at the low-dimensional bottleneck such that levels align in encoders and generative decoders, respectively implemented via hierarchies of superficial and deep pyramidal neurons. (Note: A somewhat similar proposal has been independently described by Kanai et al. (2019).) To implement predictive coding, descending messages from generative decoder networks would continuously suppress (or "explain away") ascending messages from encoders. In this coding scheme, only failed predictions from generative decoders get passed upwards through encoders, with these prediction errors continuing to rise up hierarchical levels until they can be successfully suppressed by the descending stream. These descending predictions are generated on multiple levels, both locally via recurrent dynamics, as well as on a more global basis, potentially accompanied by unique architectural features and discrete updating of integrative models (Friston et al., 2017c; Parr and Friston, 2018b). Viewed as a folded auto-encoder, these higher-level predictions would constitute a parameterization of generative decoder networks by samples from reduced-dimensionality latent feature spaces. As training proceeds, such an architecture should form increasingly predictive and sparse representations, so maximizing inferential power, while also minimizing the number of messages that need to be passed. This training for prediction and sparsification would correspond to the development of models of increasing accuracy, efficiency, and robust generalizability (Srivastava et al., 2014).

A predictive coding model of cortex would correspond to not just a single (folded) autoencoder hierarchy, but a heterarchy composed of multiple intersecting hierarchies, so enabling cortical learning systems to obtain inferential synergy through multi-modal sensory integration (McGurk and MacDonald, 1976; Eguchi et al., 2020). In terms of machine learning principles, high-bandwidth connections between association cortices could correspond to the chaining of low-dimensionality bottlenecks from multiple autoencoders, so forming an autoassociative network capable of supporting loopy belief propagation (the potential functional significance of which will be explored below). Neuroanatomically speaking, these highly connected areas would correspond to the brain's rich club networks (Heuvel et al., 2012), including the previously discussed 2D grid structures that could contribute to spatiotemporal modeling. Further, the regulation of neuronal dynamics by diffuse neuromodulator systems could be computationally understood as parameterizing inference and learning both with respect to the formation of partially disentangled features in perception, and of selecting and sculpting particular policies for enaction (e.g. dopamine as precision weighting or Kalman gain) (Parr and 
Friston, 2018a). To the degree diffuse neuromodulator systems both influence and are influenced by overall levels of message passing, these chemicals could be used to adaptively optimize generative models with context sensitivity.

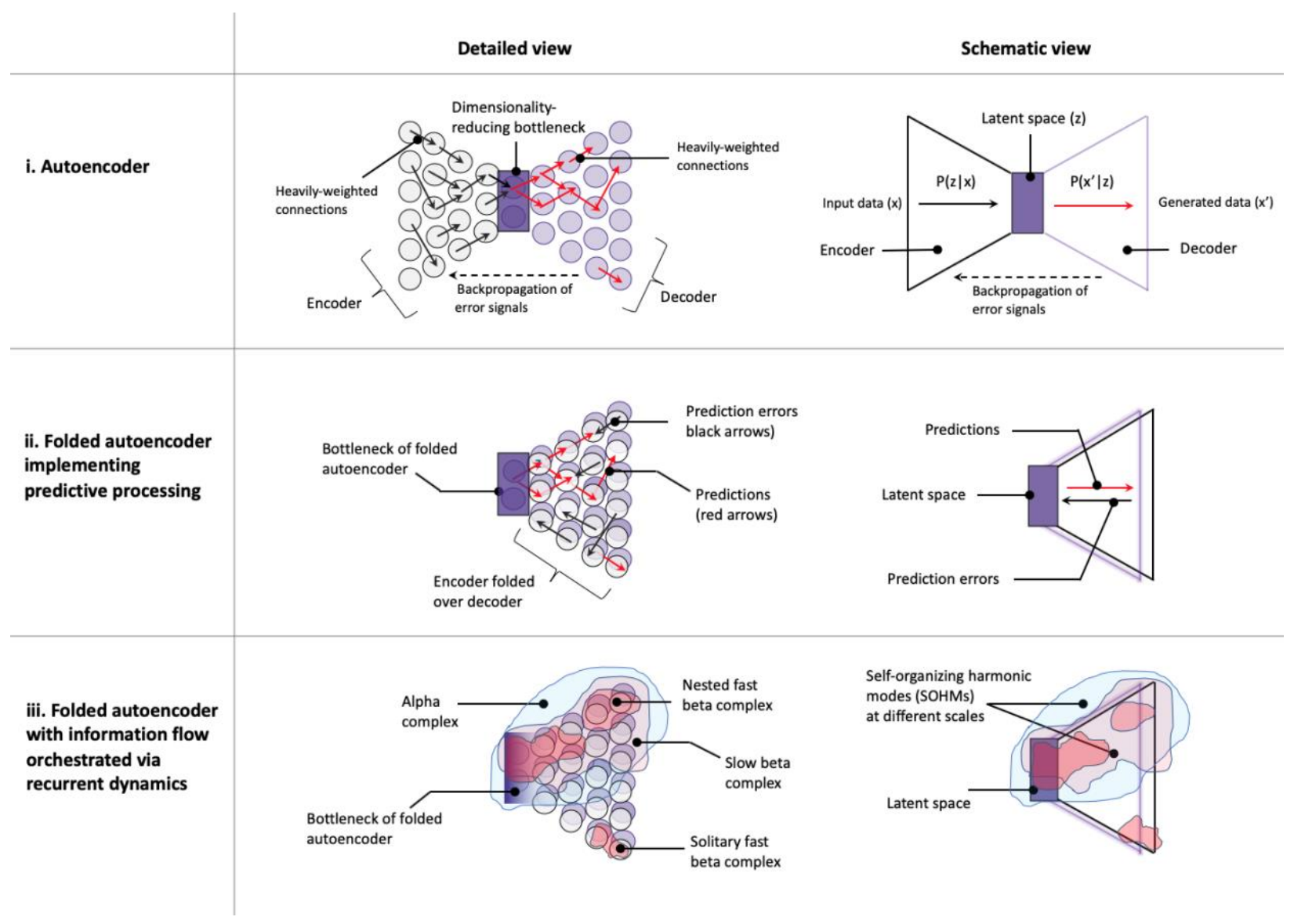

Figure 2 from “An Integrated World Modeling Theory (IWMT) of consciousness: Combining Integrated Information and Global Neuronal Workspace Theories with the Free Energy Principle and Active Inference Framework; towards solving the Hard problem and characterizing agentic causation": Sparse folded variational autoencoders with recurrent dynamics via self-organizing harmonic modes (SOHMs).

Figure 2.i: Autoencoder.

An autoencoder is a type of artificial neural network that learns efficient representations of data, potentially including a capacity for generating more complete data from less complete sources. The encoder compresses input data over stages of hierarchical feature extraction, passes it through a dimensionality-reducing bottleneck and into a decoder. The decoder attempts to generate a representation of the input data from these reduced-dimensionality latent representations. Through backpropagation of error signals, connections contributing to a more inaccurate representation are less heavily weighted. With training, the decoder learns how to generate increasingly high-fidelity data by utilizing the compressed (and potentially interpretable) feature representations encoded in the latent space of the bottleneck portion of the network. In the more detailed view on the left, black arrows on the encoder side represent connections contributing to relatively high marginal likelihoods for particular latent feature space representations, given connection weights and data. Red arrows on the decoder side represent connections with relatively high marginal likelihoods for those reconstructed features, given connection weights and latent space feature hypotheses. While these autoencoders are fully connected dense networks, particular connections are 
depicted (and associated probabilities discussed) because of their relevance for predictive processing. Note: Although the language of probability theory is being used here to connect with neurobiologically-inspired implementations, this probabilistic interpretation - and links to brain functioning — is more commonly associated with variational autoencoders, which divide latent spaces into mean and variance distributions parameterized by stochastic sampling operations in generating likely patterns of data, given experience.

Figure 2.ii: Folded autoencoder implementing predictive processing.

In this implementation of predictive processing, autoencoders are 'folded' at their low-dimensionality bottleneckssuch that corresponding encoding and decoding layers are aligned - with decoding hierarchies (purple circles) depicted as positioned underneath encoding hierarchies (gray circles). Within a brain, these decoding and encoding hierarchies may correspond to respective populations of deep and superficial pyramidal neurons (Bastos et al., 2012). In the figure, individual nodes represent either units in an artificial network - or groups of units; e.g. capsule networks (Kosiorek et al., 2019) —or neurons (or neuronal groups; e.g. cortical minicolumns) in a brain. Predictions (red arrows) suppress input signals when successfully predicted, and are depicted as traveling downwards from representational bottlenecks (corresponding to latent spaces) along which autoencoding networks are folded. Prediction errors, or observations for a given level (black arrows) continue to travel upwards through encoders unless they are successfully predicted, and so "explained away." Data observations (i.e., prediction errors) are depicted as being sparser relative to high-weight connections in the (non-folded) encoding network presented above, where sparsity is induced via predictive suppression of ascending signals. This information flow may also be viewed as Bayesian belief propagation or (marginal) message passing (Friston et al., 2017c; Parr et al., 2019b). In contrast to variational autoencoders in which training proceeds via backpropagation with separable forward and backward passes - where cost functions both minimize reconstruction loss and deviations between posterior latent distributions and priors (usually taking the form of a unit Gaussian) - training is suggested to occur (largely) continuously in predictive processing (via folded autoencoders), similarly to recent proposals of target propagation (Hinton, 2017; Lillicrap et al., 2020). Note: Folded autoencoders could potentially be elaborated to include attention mechanisms, wherein higher-level nodes may increase the information gain on ascending prediction-errors, corresponding to precision-weighting (i.e., inverse variance over implicit Bayesian beliefs) for selected feature representations.

Figure 2.iii: Folded autoencoder with information flow orchestrated via recurrent dynamics.

This row shows a folded autoencoder model of a cortical hierarchy, wherein neuronal oscillations mediate predictions - potentially orchestrated by deep pyramidal neurons and thalamic (and striatal) relays - here characterized as self-organizing harmonic modes (SOHMs). This paper introduces SOHMs as mechanisms realizing synchronization manifolds for coupling neural systems (Palacios et al., 2019), and sources of coherent neuronal oscillations and evidence accumulation for predictive processing. Depending on the level of granularity being considered, these predictive oscillations could either be viewed as traveling or standing waves (i.e., harmonics). SOHM-based predictions are shown as beta oscillations forming multiple spatial and temporal scales. These predictive waves may be particularly likely to originate from hierarchically higher levels - corresponding to latent spaces of representational bottlenecks - potentially due to a relatively greater amount of internal reciprocal connectivity, consistent information due to information aggregation, or both. SOHMs may also occur at hierarchically lower levels due to a critical mass of model evidence accumulation allowing for the generation of coherent local predictions, or potentially on account of semi-stochastic synchronization. Faster and smaller beta complexes are depicted as nested within a larger and slower beta complex, all of which are nested within a relatively larger and slower alpha complex. Note: In contrast to standard machine learning implementations, for this proposal of predictive processing via folded autoencoders (and SOHMs), latent space is depicted as having unclear boundaries due to its realization via recurrent dynamics. Further, inverse relationships between the spatial extent and speed of formation for SOHMs are suggested due to the relative difficulties of converging on synchronous dynamics within systems of various sizes; theoretically, this mechanism could allow for hierarchical modeling of events in the world for which smaller dynamics would be expected to change more quickly, and where larger dynamics would be expected to change more slowly.

Hippocampal complex and heterarchical hidden Markov models; brains as disentangling Kalman variational autoencoders 
In this view of the brain in terms of machine learning architectures, the hippocampal complex could be thought of as the top of the cortical heterarchy (Hawkins and Blakeslee, 2004; Baldassano et al., 2017) and spatiotemporally-organized memory register. As IWMT suggests, this spatial and temporal organization may be essential for coherent world modeling. With respect to grid/place cells of the entorhinal/hippocampal system (Moser et al., 2008), this organization appears to take the form of 2D trajectories through space, wherein organisms situate themselves according to a kind of simultaneous localization and mapping via Kalman (Bayesian) filtering. Anatomically speaking, this dynamic (and volatile) memory system has particularly strong bi-directional linkages with deeper portions of cortical generative models (i.e., reduceddimensionality latent feature spaces), so being capable of both storing information and shaping activity for these core auto-associative networks. Because of the predictive coding setup-and biasing via neuromodulatory value signals (Mannella et al., 2013; McNamara and Dupret, 2017) - only maximally informative, novel, unexplained observations will tend to be stored in this spatiotemporally organized memory register. Indeed, this may be one of the primary functions of the hippocampus: temporarily storing information that could not be predicted elsewhere, and then using replay to train relevant subnetworks to be more successfully predictive of likely observations.

As the hippocampus replays trajectories of the organism through space, pointers to prediction errors will be replayed in sequences, with the generative model inferring a more complete sensorium based on its training from a lifetime of experience. Computationally speaking, this setup would correspond to a Kalman variational auto-encoder (Fraccaro et al., 2017). Experientially speaking, this integration of organismic spatiotemporal trajectories with auto-associative filling-in could provide not just a basis for forming episodic memories, but also the imagination of novel scenarios (Hassabis and Maguire, 2009). Importantly, memories and imaginings can be generated by cortex on its own - given a lifetime of experience with a functioning entorhinal/hippocampal system-but medial temporal lobe involvement appears to be required for these dynamics to be shaped in novel directions that break free of past experience (Canolty and Knight, 2010; Sarel et al., 2017; Hills, 2019; MacKay, 2019).

\section{The conscious turbo-code}

Turbo-codes are used for reliably sending data over noisy channels (Berrou et al., 1993; Berrou and Glavieux, 1996), with efficiency approaching the Shannon limit, suggesting near optimality. These codes were independently discovered by the cryptography community and Judea Pearl (1982) as a method for approximate Bayesian inference via loopy belief propagation (McEliece et al., 1998). This method of extracting information from noisy signals has found a wide range of uses, including with respect to $3 \mathrm{G}$ and $4 \mathrm{G}$ wireless communication standards. Perhaps these codes were also discovered by natural selection?

IWMT proposes that turbo-coding may be implemented by reciprocal effective connectivity between auto-associated cortical hierarchies, entailing shared reduceddimensionality latent feature spaces among coupled autoencoders. Mechanistically, this would be realized by the formation of large-scale synchronous complexes as self-organizing harmonic modes (SOHMs) over connectivity backbones, some of which may entail embodied-self models (ESMs) and posterior medial visual fields in the ways described above. Algorithmically, this would correspond to the calculation of approximate joint posteriors - and maximally likely (MAP) estimates derived thereof-via loopy belief propagation. Functionally, this would 
correspond to a series of estimated world states of sufficient reliability to form bases for action selection (Vul et al., 2014). Experientially, this would correspond to the stream of consciousness. (Note: While all synchronous complexes could potentially be interpreted as engaging in turbocoding on some level of abstraction, IWMT suggests that only turbo-codes spanning multiple modalities are likely to be capable of generating conscious experiences.)

The high-bandwidth message passing required for conscious turbo-coding may be enabled by the brain's rich-club, which consumes up to $50 \%$ of cortical metabolism (Heuvel et al., 2012). However, this metabolic expense may be (evolutionarily) justified by reducing the overall number of (noisy) neuronal signal transactions required to achieve adequately reliable perceptual inference, so increasing overall efficiency, and perhaps more importantly, decreasing latencies with respect to action selection. Perhaps most importantly of all, turbo-coding over subnetworks where multiple modalities converge may enable the inferential synergy required for conscious experiences and the imagination of counterfactual scenarios (Buchsbaum et al., 2012; Schmidhuber, 2012; Pearl and Mackenzie, 2018), so facilitating a) causal reasoning, b) planning, and c) 'offline' learning (e.g. imaginative self-play).

Different frequencies may entail different kinds of information with respect to conscious turbo-codes. When beta complexes are cross-frequency phase coupled within alpha rhythms in posterior cortices, this may correspond to cross-modal message passing across the entire sensorium of the organism, organized within egocentric spatial reference frames, entailing consciousness (i.e., an experienced world). When these alpha and beta complexes are further orchestrated by theta rhythms from the entorhinal/hippocampal system and its "big loop recurrence" with frontal cortices (Koster et al., 2018), this may correspond to action-driven perception (including simulated actions), and reflective access via comparisons amongst conscious states.

Thus, turbo-coding may help to explain some of the mechanisms enabling consciousness. However, these modeling efforts may themselves have a further (circular) causal significance in that they may help to facilitate the conditions that enable them. That is, under normal circumstances, only coherent and well-evidenced world models are likely to enable loopy message passing to converge upon (approximate) posteriors, which in turn allow consciously experienced world models to arise. Developmentally, this kind of circular bootstrapping suggests that modeling capacity may increase non-linearly, potentially resulting in relatively abrupt (or punctuated) phase transitions for the evolution of consciousness (Isler et al., 2018).

In this view, consciousness emerges from an auto-associative network of coupled generative decoders, connected together to constitute a turbo-code. When message passing is forced to converge via synchrony - and where synchrony emerges from convergent message passing - this may entail maximal a posteriori (MAP) estimates as coherent/discrete vectors with maximal control in governing overall system evolution, sampled from probabilistic spatialtemporal-causal world models. Thus, consciousness (as turbo-code) may not only govern perception as Bayesian model selection, but also action selection (broadly construed to include thought as covert 'behavior'). 
i. Turbo coding between autoencoders

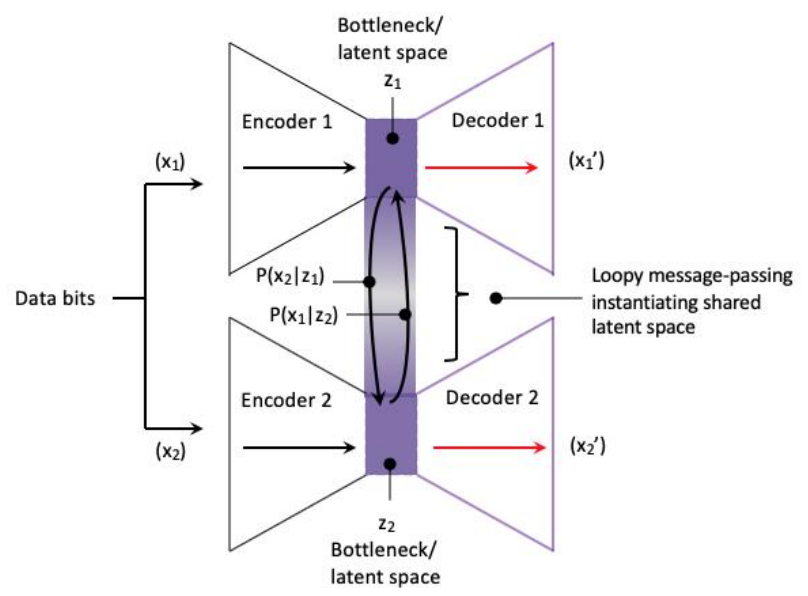

ii. Turbo coding between folded autoencoders

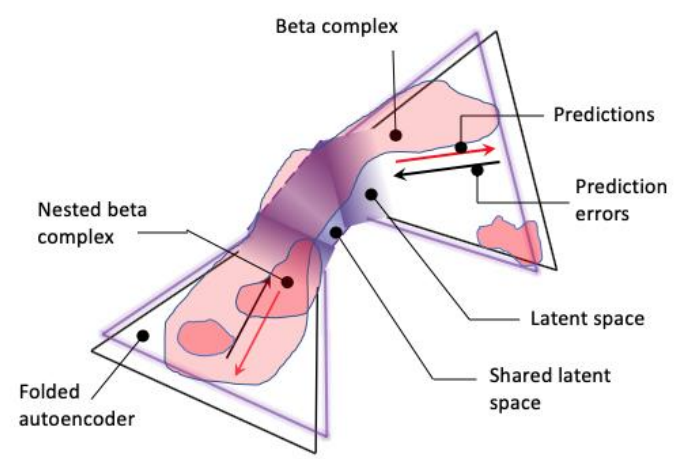

iii. Multiplexed multi-scale turbo coding between folded autoencoders

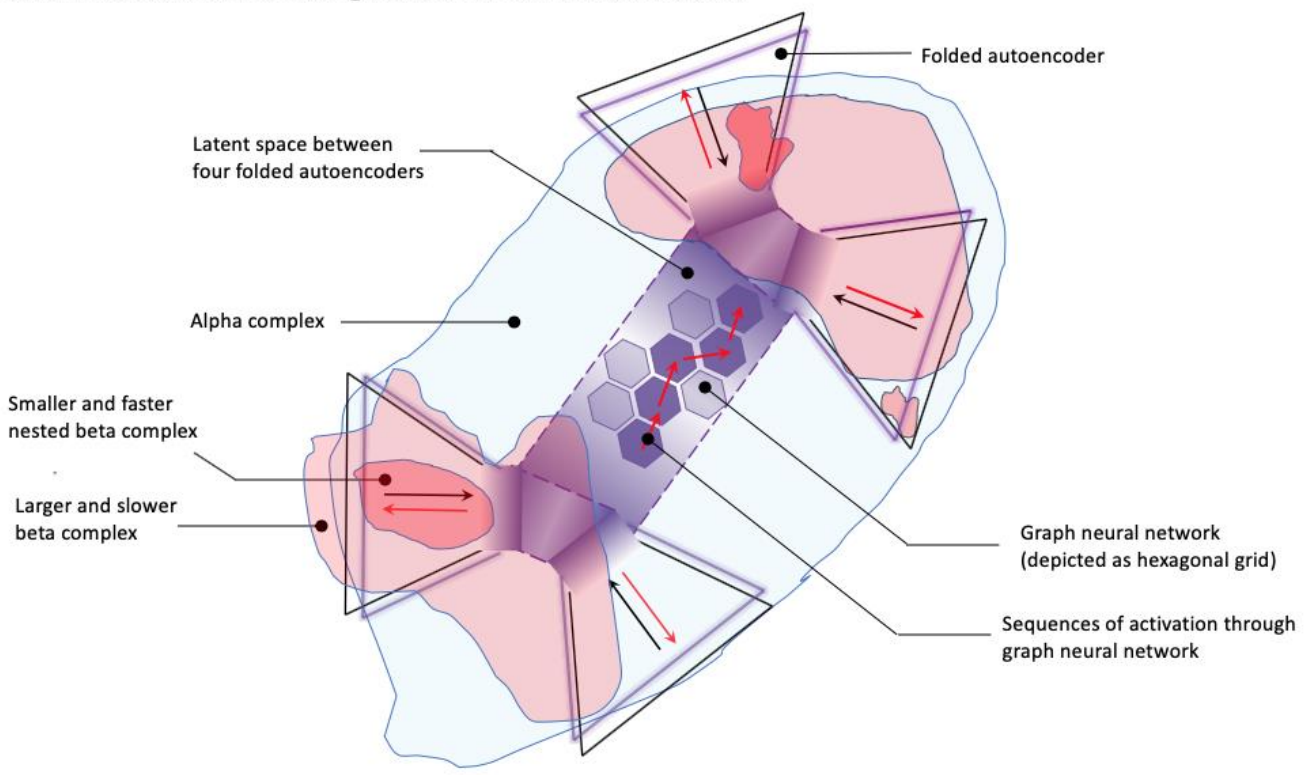

Figure 3 from “An Integrated World Modeling Theory (IWMT) of consciousness: Combining Integrated Information and Global Neuronal Workspace Theories with the Free Energy Principle and Active Inference Framework; towards solving the Hard problem and characterizing agentic causation": Cortical turbo codes

Figure 3.i. Turbo coding between autoencoders. Turbo coding allows signals to be transmitted over noisy channels with high fidelity, approaching the theoretical optimum of the Shannon limit. Data bits are distributed across two encoders, which compress signals as they are passed through a dimensionality reducing bottleneck - constituting a noisy channel - and are then passed through decoders to be reconstructed. To represent the original data source from compressed signals, bottlenecks communicate information about their respective (noisy) bits via loopy message passing. Bottleneck $\mathrm{z}_{1}$ calculates a posterior over its input data, which is now passed to Bottleneck $\mathrm{z}_{2}$ as a prior for inferring a likely reconstruction (or posterior) over its data. This posterior is then passed back in the other direction 
(Bottleneck $\mathrm{z}_{2}$ to Bottleneck $\mathrm{z}_{1}$ ) as a new prior over its input data, which will then be used to infer a new posterior distribution. This iterative Bayesian updating repeats multiple times until bottlenecks converge on stable joint posteriors over their respective (now less noisy) bits. IWMT proposes that this operation corresponds to the formation of synchronous complexes as self-organizing harmonic modes (SOHMs), entailing marginalization over synchronized subnetworks - and/or precision-weighting of effectively connected representations-with some SOHM-formation events corresponding to conscious "ignition" as described in Global Neuronal Workspace Theory (Dehaene, 2014). However, this process is proposed to provide a means of efficiently realizing (discretely updated) multi-modal sensory integration, regardless of whether "global availability" is involved. Theoretically, this setup could allow for greater data efficiency with respect to achieving inferential synergy and minimizing reconstruction loss during training in both biological and artificial systems. In terms of concepts from variational autoencoders, this loopy message passing over bottlenecks is proposed to entail discrete updating and maximal a posteriori (MAP) estimates, which are used to parameterize semi-stochastic sampling operations by decoders, so enabling the iterative generation of likely patterns of data, given past experience (i.e., training) and present context (i.e., recent data preceding turbo coding). Note: In turbo coding as used in industrial applications such as enhanced telecommunications, loopy message passing usually proceeds between interlaced decoder networks; within cortex, turbo coding could potentially occur with multiple (potentially nested) intermediate stages in deep cortical hierarchies.

Figure 3.ii. Turbo coding between folded autoencoders. This panel shows turbo coding between two folded autoencoders connected by a shared latent space. Each folded autoencoder sends predictions downwards from its bottleneck (entailing reduced-dimensionality latent spaces), and sends prediction errors upwards from its inputs. These coupled folded autoencoders constitute a turbo code by engaging in loopy message passing, which when realized via coupled representational bottlenecks is depicted as instantiating a shared latent space via highbandwidth effective connectivity. Latent spaces are depicted as having unclear boundaries-indicated by shaded gradients - due to their semi-stochastic realization via recurrent dynamics. A synchronous beta complex is depicted as centered on the bottleneck latent space — along which encoding and decoding networks are folded — and spreading into autoencoding hierarchies. In neural systems, this spreading belief propagation (or message-passing) may take the form of traveling waves of predictions, which are here understood as self-organizing harmonic modes (SOHMs) when coarse-grained as standing waves and synchronization manifolds for coupling neural systems. Relatively smaller and faster beta complexes are depicted as nested within - and potentially cross-frequency phase coupled by - this larger and slower beta complex. This kind of nesting may potentially afford multi-scale representational hierarchies of varying degrees of spatial and temporal granularity for modeling multi-scale world dynamics. An isolated (small and fast) beta complex is depicted as emerging outside of the larger (and slower) beta complex originating from hierarchically higher subnetworks (hosting shared latent space). All SOHMs may be understood as instances of turbo coding, parameterizing generative hierarchies via marginal maximum a posteriori (MAP) estimates from the subnetworks within their scope. However, unless these smaller SOHMs are functionally nested within larger SOHMs, they will be limited in their ability to both inform and be informed by larger zones of integration (as probabilistic inference).

Figure 3.iii Turbo coding between four folded autoencoders. This panel shows turbo coding between four folded autoencoders. These folded autoencoders are depicted as engaging in turbo coding via loopy message passing, instantiated by self-organizing harmonic modes (SOHMs) (as beta complexes, in pink), so forming shared latent spaces. Turbo coding is further depicted as taking place between all four folded autoencoders (via an alpha complex, in blue), so instantiating further (hierarchical) turbo coding and thereby a larger shared latent space, so enabling predictive modeling of causes that achieve coherence via larger (and more slowly forming) modes of informational integration. This shared latent space is illustrated as containing an embedded graph neural network (GNN) (Liu et al., 2019; Steppa and Holch, 2019), depicted as a hexagonal grid, as a means of integrating information via structured representations, where resulting predictions can then be propagated downward to individual folded autoencoders. Variable shading within the hexagonal grid-space of the GNN is meant to indicate degrees of recurrent activity - potentially implementing further turbo coding - and red arrows over this grid are meant to indicate sequences of activation, and potentially representations of trajectories through feature spaces. These graphgrid structured representational spaces may also afford reference frames at various levels of abstraction; e.g. space proper, degrees of locality with respect to semantic distance, abductive connections between symbols, causal relations, etc. If these (alpha- and beta-synchronized) structured representational dynamics and associated predictions afford world models with spatial, temporal, and causal coherence, these processes may entail phenomenal consciousness. Even larger integrative SOHMs may tend to center on long-distance white matter 
bundles establishing a core subnetwork of neuronal hubs with rich-club connectivity (van den Heuvel \& Sporns, 2011). If hippocampal-parietal synchronization is established (typically at theta frequencies), then bidirectional pointers between neocortex and the entorhinal system may allow decoders to generate likely patterns of data according to trajectories of the overall system through space and time, potentially enabling episodic memory and imagination. If frontal-parietal synchronization is established (potentially involving theta-, alpha-, and betasynchrony), these larger SOHMs may also correspond to "ignition" events as normally understood in Global Neuronal Workspace Theory, potentially entailing access consciousness and volitional control.

\section{Commentary on The Generative Adversarial Brain}

Gershman (2019) has presented an intriguing account of neural functioning in terms of a powerful class of generative models known as generative adversarial networks (GANs). GANs have many similar use cases to variational-encoders (VAEs) and can even be used in combination for enhanced training as in the case of VAE-GANs. In Gershman's proposal, sensory cortices act as generators which are trained via Turing learning, with frontal cortex functioning as a discriminator and source of (higher-order) consciousness.

IWMT, in contrast, suggests that predictive coding can be understood as generating (basic phenomenal) consciousness via folded VAEs in the ways described above. From this perspective, the ascending and descending streams for each modality constitute respective encoding and generative decoding networks. This is not inconsistent with Gershman's proposal, in that a sensory hierarchy as a whole can be viewed as a generative network, which relative to the entire brain may provide a VAE-GAN setup. Alternatively, the ascending stream could be interpreted as acting as a discriminator in the GAN sense, in that it is attempting to evaluate the degree to which the descending stream generates veridical images. In this view, folded autoencoders might also be understood as folded GANs, but with folds taking place at output layers of generative decoders and input layers of discriminative encoders. The ascending stream is well-poised to serve this kind of discriminative function in terms of being more directly in touch with the ground truth of sensation and the generative processes of the world, which are the ultimate referents and selection criteria for neural dynamics. This is somewhat different from Gershman's proposal, in that consciousness (as experience) would correspond to generative processes in posterior sensory areas (including multi-modal association cortices), trained via embodied-embedded interaction with the world, with frontal cortices functioning as an elaboration of the generative process in multiple ways, including conscious access via the stabilization and alteration of posterior networks, and also simulated actions and inter-temporal modeling (Ha and Schmidhuber, 2018).

However, frontal cortex could also be viewed as serving a discriminator function in terms of attentional biasing of information based on reliability (i.e., precision weighting), mechanistically achieved by altering the gain on excitatory activity from ascending ('discriminative') encoding networks. Thus, frontal cortex could provide a discriminatory function via tuning the sensitivity of ascending perceptual streams. Other non-mutually exclusive possibilities could also be envisioned for a discriminator-like role for frontal cortices:

1. Comparison with memory: "Is what I am perceiving consistent with what I have previously experienced?" This might be particularly important early in development for teaching patterns of attention that promote perceptual coherence.

2. Comparison with causal reasoning: "Is what I am perceiving consistent with what is plausible?" This is closer to Gershman's proposal, wherein failing to establish this 
discriminatory capacity could increase the probability of delusions and possibly lowered hallucination thresholds in some conditions.

3. Comparison with goal-attainment (a combination of 1 and 2): "Is what I am perceiving consistent with my normal predictions of working towards valued goals?" This could have the effect of adaptively shaping conscious states in alignment with personal (and ultimately organismic) value.

In all of these cases, frontal cortex could be viewed as being in an adversarial (but ultimately cooperative) relationship with sensory hierarchies, whose recognition densities would optimize for minimizing perceptual prediction error (i.e., what is likely to be, given data), and where frontally-informed generative densities would optimize for future-oriented (counterfactual) adaptive policy selection (i.e., what ought to be, given prior preferences). In these ways, action and perceptual hierarchies would compete with respect to the ongoing minimization of free energy, while at the same time being completely interdependent for overall adaptive functioning, with both competitive and cooperative dynamics being required for adaptively navigating the world via action-perception cycles. An interesting hybrid of competitive and cooperative dynamics may be found in "learning to learn" via creative imagination and play (including selfplay), in which learners may specifically try to maximize surprise/information-gain.

With respect to frontal predictions, these could potentially be broken down according to a 3-fold factorization:

1. A ventral portion representing affectively-weighted sensory outcomes associated with various actions.

2. A dorsal portion representing forward models for enacting sequences that bring about desirable outcomes.

3. A recurrent anterior pole portion that mediates between affect and action selection via its evolving/bifurcating/non-linear attractor dynamics (Izquierdo-Torres and Bührmann, 2008; Izquierdo et al., 2013; Tani, 2016).

(1) and (2) could be frontal analogues to the "what" and "where" pathways for vision (Pezzulo et al., 2018; Parr et al., 2019c) — with macroscale connectivity reflecting these functional relationships - except here we are dealing with (1) what-where (via coupling with the hippocampal complex) and (2) how-where (via coupling with the parietal lobes). Taken together (which is how these systems are likely to work under most circumstances), these different parts of frontal cortices could all be understood in a unified sense as implementing policy selection via predictions and precision-weighting.

Gershman further argues that predictive coding can be viewed as an efficient way of passing predictions up the cortical hierarchy while removing redundant information. This is consistent with proposals in which the descending stream is interpreted as constituting a means for communicating the backwards propagation of error signals to apical dendrites in cortical layer 1 (Richards et al., 2019). Although this is the opposite of the way predictive coding is normally understood, with prediction errors being communicated via the ascending stream, these accounts could potentially be reconciled if we understand perception as involving a circularcausal process of iterative Bayesian model selection. When we consider the capacity for looping effects in networks on the scale of nervous systems - for even the largest deep learning systems, the number of parameters is dwarfed (for now) by those found in a cubic millimeter of cortexwith potentially multiple levels of qualitatively different 'beliefs' (e.g. unconscious sensorimotor, conscious embodiment, and implicit schemas), then it can be difficult to straightforwardly interpret the flow of inference in terms of a clear distinction between predictions and prediction 
errors. However, we would also do well to not be overly ecumenical with respect to this potential reconciliation, as classical accounts of predictive coding induce sparsity on multiple levels, so creating many highly desirable computational properties such as energy efficiency, robustness, and sensitivity to coincidence detection (Srivastava et al., 2014; Ahmad and Scheinkman, 2019). As such, framing the descending stream as a backpropagation signal may be an account that is both misleading and impoverished with respect to biological realities.

In terms of the potential complexity of cortical generative models, we may want to think of at least three coupled systems that are ultimately integrated as parts of a unified control hierarchy, but which can temporally evolve independently:

1. Unconscious/preconscious lower-level sensorimotor hierarchies with fast fine-grained dynamics for coupling with the environment (Tani, 2016).

2. Conscious mid-level sensorimotor representations with more coarse-grained spatial and temporal dynamics (Prinz, 2017).

3. Higher-level abstract re-representations over recognition and generative densities, with unconscious/preconscious dynamics (Arese Lucini et al., 2019), and which may bidirectionally couple with lower and middle levels.

In these ways, we could potentially dissect the brain into multiple competing and cooperating generative models, whose synergistic interactions may be productively considered as implementing GAN-type setups. Very speculatively, it may even be the case that perceptionaction cycles, hemispheric interactions, and interpersonal communication could all be understood as implementing CycleGAN-like dynamics. That is, to what extent could relationships between hemispheres and individuals be analogous to a paired GAN setup, where each system may evaluate the output of the other, so promoting the formation of usefully disentangled representations of features in reduced dimensionality latent spaces (Thomas et al., 2017, 2018), thereby promoting controllability and combinatorial power in imagination? These are only some of the many ways that Gershman's intriguing proposal of a "generative adversarial brain" may lead to innovative directions for trying to understanding functional relationships within and between minds.

\section{IWMT Implemented: Towards Conscious Artificial Intelligence?}

The figure below provides a depiction of the human brain in terms of phenomenological correspondences, as well as Marr's computational (or functional), algorithmic, and implementational levels of analysis. On the computational level, various brain functions are identified according to their particular modal character, either with respect to perception (both unconscious and conscious) or action (both unconscious and potentially conscious, via perceptual generative models). On the algorithmic level, these functions are mapped onto variants of machine learning architectures (e.g. autoencoders and generative adversarial networks, graph neural networks, recurrent reservoirs and liquid state machines), organized according to their potential realization by various systems in the brain. On the implementational level, realizations of algorithmic processes are depicted as corresponding to flows of activity and interactions between neuronal populations, canalized by the formation of self-organizing harmonic modes (SOHMs) as metastable synchronous complexes. [Note: The language of predictive processing is used here to help provide bridges to the algorithmic level, and also because implementations always require some kind of coarse-graining in order to be describable; 
descriptions such as vector/tensor fields and attracting manifolds could have alternatively been used in order to remain agnostic as to which algorithms may be entailed by physical dynamics.]

A phenomenological level is specified in order to provide mappings between consciousness and these complementary/supervenient levels of analysis. These modal depictions are meant to connotate the inherently embodied nature of experience, but not all images are meant to correspond to the generation of consciousness. That is, it may be the case that consciousness is solely generated by the posterior medial cortices, parietal cortices, and temporoparietal junction as respective visuospatial (cf. consciousness as projective geometric modeling), somatic (cf. embodied cognition and intermediate level theory), and attentional/intentional phenomenology (cf. Attention Schema Theory). These brain systems are both depicted as associated with alpha frequencies on the implementational level, and as graph neural networks on the algorithmic level.

Graph neural networks (GNNs) are identified as a potentially important machine learning architectural principle, largely due to their efficiency in emulating physical processes, and also because the message passing protocols during training and inference may have correspondences with loopy belief propagation and turbo codes suggested by IWMT. Further, grid graphspotentially hexagonally organized, possibly corresponding to cortical macrocolumns, with nested microcolumns that may also be organized as hexagonal grid GNNs, or "capsule networks") — are adduced for areas contributing to quasi-Cartesian spatial modeling (and potentially experience), including the posterior medial cortices, dorsomedial and ventromedial prefrontal cortices, and the hippocampal complex. With respect to AI systems, such representations could be used to implement not just modeling of external spaces, but of consciousness as internal space (or blackboard), which could potentially be leveraged for reasoning processes based with correspondences to category theory, analogy making via structured representations, and possibly causal inference. Neuroimaging evidence suggests these grids may be dynamically coupled in various ways, with these aspects of higher-order cognition being understood as a kind of generalized navigation/search process. A further GNN is speculatively adduced in parietal cortices as a mesh grid placed on top of a transformed representation of the primary sensorimotor homunculus (cf. body schemas for the sake of efficient motor control/inference), which is here suggested to have some correspondence/scaling to the body as felt from within, but which may be further morphed to correspond with externally viewed embodiments (potentially both resulting from and enabling "mirroring" with the bodies of other agents for the sake of coordination and inference). This translation into an alternative coordinate system is also suggested to provide more effective couplings (or information sharing) with the potentially topographically organized representations in posterior medial cortices. [Note: Considering the highly specific and peculiar nature of these suggestions, evidence for such organizational principles would constitute strong evidence in favor of IWMT.] Temporoparietal junction is depicted as containing a ring-shaped GNN to reflect a further level of abstraction and hierarchical control over action-oriented body schemas, whose sparse features may contribute to ineffable/elusive and soul-like properties of self-consciousness, functionally entailing vectors/tensors over attentional/intentional processes.

Frontal homologues to posterior GNNs are also depicted, which may provide a variety of higher-order modeling abilities, including epistemic access for extended/distributed selfprocesses and intentional control mechanisms. These higher-order functionalities may be achieved via frontal cortices being more capable of future-oriented generative modeling with greater temporal depth and counterfactual richness, due to being situated at higher levels within 
hierarchically-organized networks, and also more distally located relative to primary sensory cortices, so affording dynamics that are more decoupled from immediate sensorimotor contingencies. Further, these frontal control hierarchies afford multi-scale goal-oriented behavior via bidirectional effective connectivity with the basal ganglia (i.e., winner-take-all dynamics and facilitation of sequential operations) and canalization via diffuse neuromodulator nuclei of the brainstem (i.e., implicit policies and value signals). Finally, the frontal pole is described as a highly non-linear recurrent system capable of shaping overall activity via bifurcating capacities - with potentially astronomical combinatorics - providing sources of novelty and rapid adaptation via situation-specific attractor dynamics. [Note: IWMT proposes frontal cortices may only indirectly contribute to consciousness via influencing dynamics in posterior cortices.] Extremely speculatively, functional analogues for ring-shaped GNN salience/relevance maps may potentially be found in the central complexes of insects and the tectums of all vertebrates, although it is unclear whether those structures would be associated with any kind of subjective experience. Even more speculatively, if these functional mappings were realized in a humanmimetic/neuromorphic/"psychomorphic" AI, then it may have both flexible general intelligence and consciousness. In this way, this figure can be considered to be a sort of pseudocode for potentially conscious (partially human-interpretable) AGI with "System 2" capacities. 


\section{Figure 4: IWMT Implemented}

Computational Level
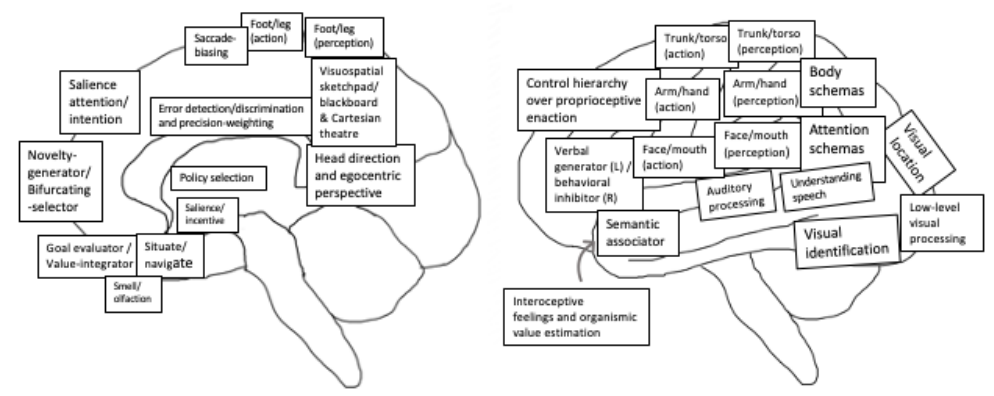

Phenomenological Level
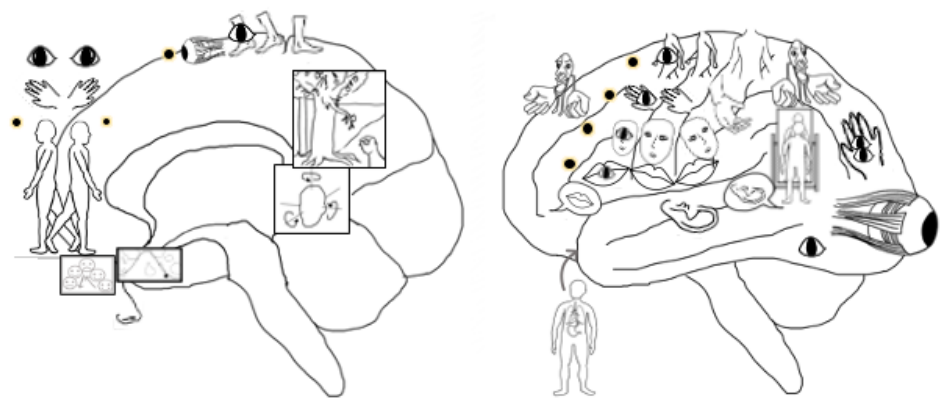

Neural Algorithmic Level*
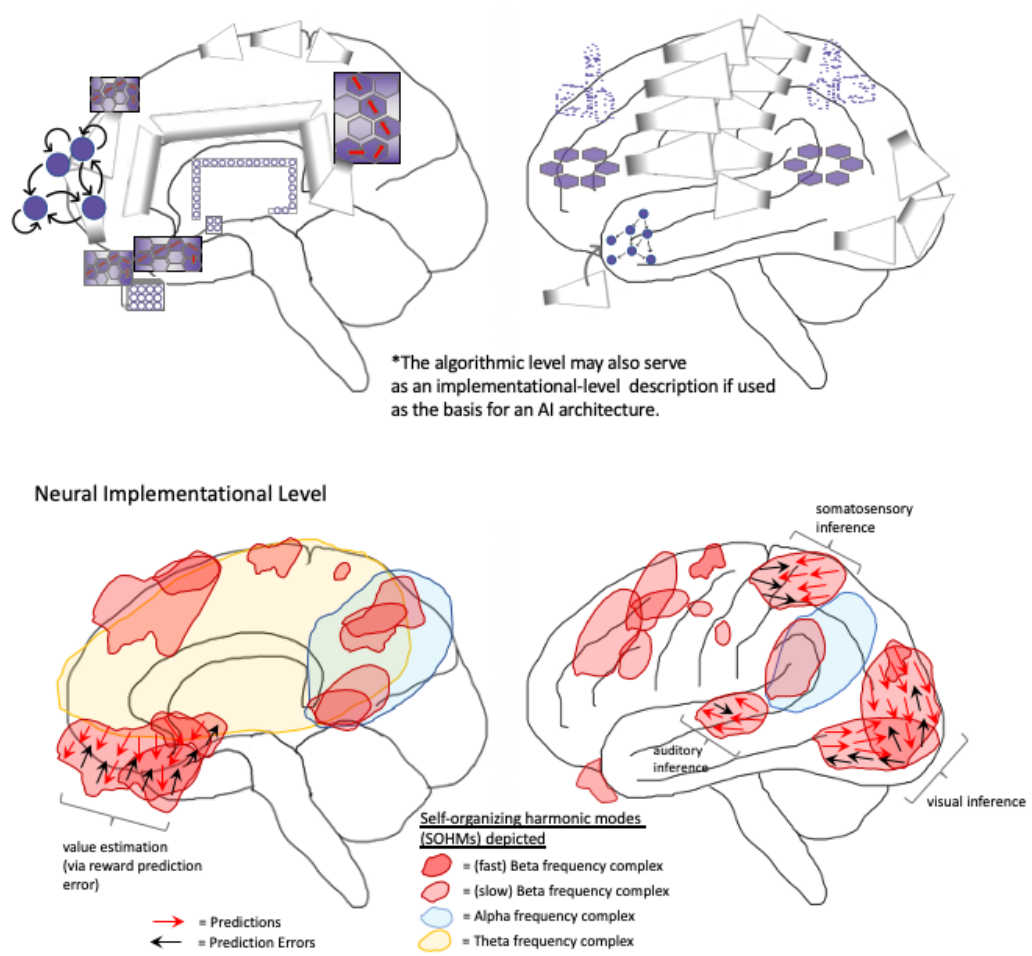


\section{Future directions for IIT and GNWT}

IWMT proposes that FEP-AI can be used as a framework for synergistically integrating leading theories of consciousness, specifically focusing on IIT and GNWT. Below we will discuss some of the ways in which these theories may be further advanced through their integration.

\section{A review of IIT terminology}

The IIT formalism begins by choosing a candidate system — as substrate for consciousness or maximal complex with irreducible cause-effect power over itself-and then identifying the intrinsic cause-effect structure of that (proto-)system as a set of elements. The cause-effect structure for (and definition of) a system is composed of all maximally irreducible cause-effect distinctions (i.e., "concepts", or "MICE"), which in recent developments within IIT have been extended to include relations between concepts/distinctions (Haun and Tononi, 2019). In this way, each and every intrinsically existing system is defined by a single (maximally irreducible) cause-effect structure, composed of at least one and possibly many distinctions/concepts/MICE. The cause-effect structure and corresponding Phi value can be computed for any candidate system, but only candidates that maximize integrated information (as Phi, or self-cause-effect power) exclusively qualify as intrinsically existing systems. These maximal complexes (of experience) are referred to as MICS in IIT 3.0, and are hypothesized to correspond to the physical substrates of consciousness.

In this way, the interrelationships between the conceptual entities of IIT for analyzing potentially conscious systems may be summarized as follows:

1. Elements: Physical constituents of a system that can be manipulated and observed, which may or may not contribute to a system's cause-effect structure.

2. Mechanisms: Individual elements or sets of elements that have irreducible cause-effect power by virtue of constraining both past and future states of other elements within a candidate system.

3. Cause-effect repertoire: Probability distributions over both past and future states for a mechanism, given its present state.

4. Concepts/distinctions: Mechanisms and their associated phi values and the intrinsic information (as distinctions) specified over their purviews with respect to repertoires of causes and effects within a candidate system, understood as an ability to maximally (irreducibly) constrain past and future states, given present states (i.e., MICE, or maximally irreducible cause effect repertoires).

5. Cause-effect structure: The set of all concepts/distinctions for all mechanisms within a system.

6. Conceptual structure: All cause-effect repertoires within a candidate system (which may potentially be reducible to simpler systems), so including all the intrinsic (cause-effect) information from the mechanisms of which a system is composed.

7. Complex: Candidate system with maximal Phi across all possible system definitions, existing for itself intrinsically, corresponding to the physical substrate of consciousness.

8. MICS: The maximally irreducible cause-effect structure entailed by a complex, corresponding to "what it is like" to be that intrinsically existing set of mechanisms, 
composed of $M I C E$, which correspond to the individual phenomenal distinctions within experience.

In this way, IIT begins from axioms regarding the nature of consciousness, postulates corresponding mechanisms with those properties, and then formally analyzes candidate systems from a system-internal perspective in order to identify sets of mechanisms with maximal claim to intrinsic existence as nexuses of self-cause-effect-power (i.e., integrated information). A maximal complex is suggested to constitute a physical substrate of consciousness, entailing a MICS as the totality of experience (as concept) unfolding at a particular temporal and spatial grain, within which particular qualitative distinctions can be identified (i.e., MICE).

IWMT agrees with this process for identifying maximally explanatory systems and even physical substrates of consciousness, except a MICS would entail subjectivity if (and only if) it corresponded to a joint probability distribution (or maximal estimate derived thereof) from a generative model with spatial, temporal, and causal coherence for system and world. In this Bayesian/FEP-AI interpretation of IIT, MICE repertoires would correspond to particular factorizations of generative models, which would have phenomenal content by virtue of reflecting sensorimotor states for embodied-embedded agents. That is, the reason there may be "something that it is like" to be such a generative model, is because neural dynamics are coupled to (or entrained with) sensors and effectors (which are in turn coupled to system-world dynamics), so providing means of modeling spatiotemporally (and causally) coherent patterns in system and world. In this way, spatiotemporal (and causal) coherence for both system and world allows for the possibility of phenomenal consciousness through the alignment/representation of (or generalized synchrony between) those coherences.

\section{Modules and workspaces as complexes of integrated information}

GNWT describes how global workspaces allow otherwise isolated specialist modules to exchange information. However, the dynamics by which modules and workspaces interact remain poorly understood. IIT describes how complexes of effective connectivity can have varying degrees of cause-effect power upon themselves. However, the functional relationships between complexes of integrated information remain poorly understood. With FEP-AI as an integrative framework, it may be possible to combine GNWT's emphasis on function and IIT's emphasis on dynamics in mutually informative ways. A potentially promising avenue is to apply IIT's analytic approaches to modules and workspaces as complexes with varying degrees of irreducible self-cause-effect power, including with respect to the ways integrated information varies over the course of cognitive cycles.

Some justification for this approach can be found by evaluating modules and workspaces in terms of the axioms of IIT:

1. Intrinsic existence

a. Modules have cause-effect power upon themselves; modules depend upon workspaces in order to have cause-effect power upon each other.

b. Workspaces have cause-effect power upon themselves and the modules with which they couple.

2. Composition

a. Modules have internal structure by which they exert cause-effect power on themselves, as well as other modules via workspaces. 
3. Information

b. Workspaces have internal structures by which they exert cause-effect power upon themselves, the compositions of which depend on the modules with which they couple (and so modify their internal compositions).

a. Modules have particular cause-effect structures that differentiate their specific compositions from other possible configurations; modules depend on workspaces to share this intrinsic information with each other.

b. Workspaces have particular cause-effect structures that specify particular largescale state compositions, which inform and are informed by the modules with which they couple in the context of cognitive cycles of perception and action selection, so acting as large-scale systemic causes.

4. Integration

a. Modules specify unified cause-effect structures that are irreducible to subcomponents.

b. Workspaces specify unified cause-effect structures that are irreducible to sub5. Exclusion components, including the modules with which they couple.

a. Modules specify particular cause-effect structures whose degree of intrinsic irreducibility evolves over particular spatial and temporal grains, depending on their ability to couple with each other and workspaces.

b. Workspaces specify particular cause-effect structures whose degree of intrinsic irreducibility evolves over particular spatial and temporal scales, with degrees of modularity and quality of communities depending on both internal and external dynamics.

Thus, both modules and workspaces can be viewed as constituting complexes of integrated information with varying amounts of irreducible self-cause-effect power (phi). The extent to which modules have more or less phi would specifically depend on the phase of cognitive cycles. Specifically, if "ignition" events correspond to the breakdown of local modularity via the formation of larger complexes of effective connectivity, then we would expect the relative phi for modules and workspaces to vary in an inverse fashion. IIT might view this changing modularity as trading off consciousness level between modules and workspaces, with separate modules entailing consciousness when they represent phi maxima, but with these consciousnesses being replaced with a single consciousness when workspace dynamics are present. IWMT and GNWT, in contrast, would only view large-scale workspaces as being capable of supporting conscious experiences.

IIT, in contrast to GNWT, does not view consciousness as corresponding to a global workspace, but only a posterior "hot zone" in constituting a phi maximum (Boly et al., 2017). The involvement of frontal cortices may be important for establishing more widespread availability and instantiating workspace dynamics of a more global nature, but according to IIT, these would not themselves represent the physical substrate of consciousness. IWMT agrees with IIT that basic phenomenality likely centers on posterior cortices, and IWMT also agrees with GNWT that frontal cortices are likely crucial for enabling access and autonoetic consciousness.

However, IWMT disagrees with IIT a given module would necessarily be conscious if it constitutes a complex that maximize integrated information (Phi). Rather, a module may be conscious only if they are capable of entailing integrated models with spatial, temporal, and causal coherence for an embodied system and its relationships to the world in which it is 
embedded. Given the previously discussed properties of posterior medial cortices, it is possible that synchronous activity within posterior hot zones may represent an instance of a (large) module being conscious when not participating in global workspace dynamics via the frontal lobes. However, this could also be viewed as a primarily semantic argument, as a complex capable of synergistically integrating information across occipital, temporal, and parietal cortices could reasonably be said to be functioning as a kind of 'global' workspace. Perhaps some disputes between GNWT and IIT may be partially resolved by attempting to be more precise about how widespread integration must be to 'count' as global.

\section{Cognitive cycles and fluctuating substrates of consciousness?}

In mammals, "posterior hot zones"(Boly et al., 2017) may be both necessary and sufficient for generating consciousness (as integrated world modeling), and these (both competitive and cooperative) attractor-formation processes may tend to be strictly dominated by dynamics within posterior association cortices. However, by coupling with posterior cortices, frontal cortices could help influence the specific compositions of maximal complexes on their timescales of formation. That is, frontal cortices may be able to influence posterior attracting networks before maximal coherence/integration is achieved, so defining spatial and temporal grains for qualia generation, enabling intentional control of attention, working memory, and action selection. When this effective coupling involves driving of frontal cortices by posterior complexes, this information may also be made more globally available for the sake of higherorder modeling. In these ways, IWMT is also in agreement with GNWT regarding the importance of frontal network hubs, although this may be the case for conscious access, rather than the more posterior-located processes that generate a coherent stream of experience.

These hypotheses could potentially be tested via transcranial magnetic stimulation applied at different phases of cognitive cycles (Madl et al., 2011; Sasai et al., 2016) in which (possibly theta-coupled) alpha rhythms may alternate across frontal and posterior cortices, assessing whether intervention influences different kinds of either implicit (e.g. via perturbation complexity index (PCI) methods) or explicit modeling. Alternatively, evoked complexity could be time-stamped to endogenous potentials as a measure of different kinds of integrative complexity, as in intriguing studies from the psychedelic neuroscience literature (Schartner et al., 2017). While the PCI can potentially be explained without appealing to IIT, it can nonetheless be used as a proxy for integrated information. If GNWT and IIT are compatible in the ways suggested by IWMT, then PCI should be higher during periods where workspace dynamics are present. This could potentially be tested by timing the TMS pulse to coincide with ignition events during which large scale integration occurs. If this were found to not be the case, it could potentially falsify IWMT.

Perhaps relatedly, an unresolved issue within IWMT is whether consciousness (as experience) corresponds to a series of discrete "snapshots" (Crick and Koch, 2003; Madl et al., 2011; Herzog et al., 2016), like a flipbook or sequential frames in a cartoon/comic (Ha and Schmidhuber, 2018), or whether such discretization reflects the process of consciously accessing — or sampling from, as in inference via Markov chain Monte Carlo (Gershman, 2019) - an otherwise continuous stream of experience. IWMT's account of synchronous complexes as corresponding to turbo coding over coupled autoencoders suggests that consciousness could either be understood as flows of inference via traveling waves on a finegrained level, or as self-organizing harmonic modes (SOHMs) when coarse-grained according to 
the scales at which various forms of functional closure are achieved (Joslyn, 2000; Chang et al., 2019), including those which would allow for the kinds of higher-order cognition involved in conscious access, self-awareness, forms of meta-awareness, acting with awareness, and planning.

In terms of the machine learning models described above, ignition events could potentially be viewed as semi-stochastic sampling from latent spaces, used by variational autoencoders to parameterize generative models in creating novel combinations of features. If these samples are biased according to histories of reward learning, then this would correspond to neural dynamics (including those entailing consciousness) being driven in directions that are most likely to realize organismic value, given the data of experience. In this way, it could be the case that ignition events themselves generate consciousness as a series of "snapshots," or maximal a posteriori (MAP) estimates from nervous systems viewed as generative models. Alternatively, it could be the case that ignition events correspond to a source of vectors that parameterize world models that evolve through more continuous updating.

That is, the seemingly continuous nature of the stream of experience could be illusory, actually corresponding to a series of MAP estimates realized by the turbo coding of ignition events, corresponding to a parameterization of sampling operations, with cortical hierarchies functionally understood as coupled variational autoencoders. Or, iteratively forming these largescale attracting-states may instead be a highly efficient (and potentially optimal) means of realizing globally coherent/integrated inference, where organizing behavior based on a series of estimates has been demonstrated to also be highly efficient from a decision-theoretic perspective (Vul et al., 2014). All of these perspectives may be accurate, except with respect to different aspects of experience unfolding on different scales. That is, while frontal-mediated conscious access may be discrete, posterior-generated basic phenomenal consciousness may truly be more like a continuous stream of entangled inferences, whose - potentially shockingly limited (Chater, 2018)_richness overflows awareness.

IWMT currently does not have a definitive prediction as to whether the prefrontal cortices ever represent a physical substrate for consciousness as suggested by GNWT. While a "posterior hot zone" may provide both necessary and sufficient conditions for generating experience as suggested by IIT, it is unclear that frontal cortices ought to be considered as separate from these generative processes, particularly during ignition events in which large-scale frontoparietal complexes are observed. Alternatively, it may be the case that frontal cortices are incapable of significantly driving the heavily entangled internal dynamics of posterior cortices on the timescales at which integration occurs, where posterior-centered inter-relations may have enough causal density to establish functional closure with respect to the processes generating coherent (and so experienceable) world models. Considerations of developmental necessity may also be relevant to debates between IIT and GNWT regarding the neural substrates of consciousness. Frontal cortices may potentially be necessary for the initial development of basic phenomenal consciousness, but not for its continued realization after sufficient experience. That is, frontal cortices may be essential for bootstrapping phenomenal consciousness via the construction of coherent world models, but once developed, these experience-generating capacities - but probably not conscious access, contrary evidence notwithstanding (Bor et al., 2017) - may be preserved even with complete disruption of these initially necessary enabling conditions.

Yet another possibility is that frontal cortices may themselves have enough integrative capacity over requisite sources of information that they represent sufficient substrates of consciousness on their own, potentially offering a source of predictions for what posterior 
cortices are likely to experience in the future (Knight and Grabowecky, 1995; Ha and Schmidhuber, 2018; Wang et al., 2018). This hypothesis of forward-looking PFCs would be consistent with their roles in action selection and motor control through predicting the sensory consequences of movement (Adams et al., 2013). However, for frontal cortices to themselves generate experience, IWMT would require sufficiency with respect to establishing perspectival reference frames with spatiotemporal and causal coherence. Regardless of whether or not frontal cortices are considered to be directly part of subnetworks generating consciousness, the nature of subjective experience will likely heavily depend on their involvement in the ways emphasized by GNWT and higher order theories (Brown et al., 2019; Shea and Frith, 2019). That is, while (very difficult to test) dissociations may be expected with respect to phenomenal consciousness being possible without conscious access, the qualities of experience will depend on their multi-scale interactions with higher order cognitive processes. For example, the act of introspecting will substantially change the nature of what is (a)perceived (e.g. attention; Sperling phenomena) (Manning et al., 2012).

Some open questions:

- Does consciousness evolve more via meta-stable standing wave attractors (Deco and Kringelbach, 2016; Atasoy et al., 2018; Wens et al., 2019), or are dynamics better understood as a continuous flow of traveling waves along the stream of experience through time (Muller et al., 2018; Zhang et al., 2018), but with periods of large-scale bifurcation entailing discrete updating of beliefs (Parr and Friston, 2018b)?

- What is the temporal extent of different kinds of ignition events, and what is the extent of synchrony at different points in time?

- Is peak ignition still sufficiently desynchronized that integration and information remain synergistically balanced (Marshall et al., 2016), potentially accompanied by critical dynamics (Carhart-Harris, 2018; Atasoy et al., 2019)?

- Could organisms be conscious leading up to and after peak ignition, but not at peak ignition?

- Could measures related to transfer entropy or PCI (Massimini et al., 2012; Schartner et al., 2017) be used to characterize the degree to which different parts of the brain are integrated at different stages of cognitive cycles?

- To what extent are large-scale events from frontal loci-whether exogenously or endogenously stimulated — capable of driving complex dynamics within posterior loci during ignition events?

\section{Mechanisms for integration and workspace dynamics}

IWMT views ignition events in terms of the formation of self-organizing harmonic modes (SOHMs), entailing message passing in nervous systems understood as Bayesian belief networks. In this way, the formation of any meta-stable synchronous complex is viewed as both an ignition event and establishment of a kind of workspace, regardless of whether or not the frontal lobes and 'global' access is achieved. In all cases, SOHMs are hypothesized to entail loopy belief propagation and marginalization over effectively connected subnetworks. In the case of small ensembles synchronized at fast gamma frequencies, SOHMs may contribute to the communication of prediction errors up cortical hierarchies (Bastos et al., 2012; Scheeringa and Fries, 2019) via quantized packets of information (as sufficient/summary statistics), so establishing marginal message passing regimes (Parr et al., 2019b). In the case of large 
ensembles synchronized at beta, alpha, and theta frequencies, SOHMs may allow for large-scale updating of beliefs and sources of integrative predictions from deeper portions of generative models.

Considering their potential central role for driving neural evolution, the dynamics by which synchronous complexes form is worth considering in detail. Some recent promising work in this direction can be found in FEP-AI, in terms of synchronous dynamics emerging through collaborative inference among interacting oscillators (Palacios et al., 2019). This kind of coordination in FEP-AI is often described in terms of the near ubiquitous phenomenon whereby systems minimize free energy through generalized synchrony (Strogatz, 2012; Kachman et al., 2017), as first demonstrated by Huygens with respect to pendulum clocks (Willms et al.; Oliveira and Melo, 2015). Here, I will provide an informal sketch of how SOHM-formation might proceed and the potential functional consequences that may result from these processes:

1. Let us consider a set of neuronal oscillators that are initially maximally desynchronized, but which gradually acquire a shared absorbing rhythm.

2. If neuronal oscillators happen to interact while phase-aligned, then they will be more likely to be able to drive activity due to stimulation happening within windows wherein temporal summation is possible.

3. If reciprocal connectivity is present between phase-aligned neuronal oscillators, then there is a potential for positive feedback and self-sustaining rhythmic activity.

4. In this way, initial locally synchronized ensembles may be able to spread synchronous organization as the stability of their rhythmic activity provides opportunities for additional phase-aligned oscillators to become entrained to the absorbing rhythm.

5. However, this potential for positive feedback must be accompanied by negative feedback mechanisms (e.g. GABAergic interneurons) to maintain adaptive exploration of state spaces (Friston et al., 2012a), and also to avoid the kinds of explosive percolation events observed in clinical conditions like epilepsy (Bartolomei and Naccache, 2011; D'Souza and Nagler, 2015; Kinouchi et al., 2019).

6. During periods of minimal synchronization, we may expect synchronizing ensembles to be maximally sensitive to signals at any phase (Lahav et al., 2018), but with minimal abilities to drive coupling systems.

7. During periods of maximum synchronization, we may expect synchronizing ensembles to be maximally sensitive to phase-aligned signals - as well as minimally sensitive to nonphase-aligned signals - and with maximal abilities to drive coupling systems.

8. During intermediate periods where synchronization dynamics are accumulating, we may expect sensitivity to a greater diversity of signals, with a potential capacity for mutual influence between coupling systems during this bifurcation window.

With respect to belief propagation in Bayesian networks, all of this could potentially be understood as a means of enabling and constraining belief propagation, influencing which messages will be likely to be exchanged on what timescales.

In terms of mesoscale and macroscale neuronal dynamics, we might expect large-scale SOHMs to be particularly likely to form in proximity to rich-club hubs of the brain with their high degrees of reciprocal connectivity. Further, as previously discussed, embodied self-models (ESMs) may tend to form backbones of effective connectivity and robust sources of synchronizing dynamics. Within these highly interconnected systems, signals may be particularly likely to undergo positive feedback amplification, where this explosive signal transduction may be able to temporarily form synchronous complexes capable of integrating information from 
across the brain and then propagating (or "broadcasting") this information to the rest of the network as Bayesian beliefs (or priors in predictive coding).

Speculatively, in terms of generalized synchrony, direction of entraining influence may potentially switch between peripheral and core networks before and after critical ignition events. That is, peripheral sensory hierarchies may asymmetrically entrain deeper levels with core connectivity, seeding them with ascending prediction errors, communicated via driving inputs at gamma frequencies. In this way, Bayesian model selection would be driven via a process of differential seeding of core states via competition (and cooperation) amongst neuronal coalitions entailing hypotheses regarding latent causes of sensory observations. Then, these discretely updated core states from deep in the heterarchy could be used to asymmetrically drive peripheral networks. According to IWMT, these core inferences would be communicated at beta frequencies for specific predictions, alpha frequencies for predictions integrated within egocentric reference frames, and theta frequencies for predictions shaped by particular actions (broadly construed to include mental acts such as attentional fixations (Parr et al., 2019a; Safron, 2019d)). Thus, SOHMs and the processes by which they form may function as complexes of integrated information and sources of workspace dynamics, so implementing Bayesian model selection on multiple levels. This multi-level selection-which may also be understood in terms of neural Darwinism and dual-phase evolution (Paperin et al., 2011)—may proceed simultaneously over multiple scales, with both global serial and local parallel integration being implemented by SOHMs of varying spatial (and temporal) extents.

It is worth noting that this proposal does not depend on any given account of predictive processing being accurate. For example, it may be the case that descending modulatory inputs at slower frequencies do not necessarily involve predictive explaining away, but could instead be used to allow sensory observations to ascend with more feedforward driving (Heeger, 2017) which would not be incompatible with an interpretation of attending based on precision weighting (i.e., Kalman gain) — as may be the case with respect to theta-gamma cross-frequency phase coupling (Canolty et al., 2010; Buzsáki and Watson, 2012) or zero-lag phase synchronization (Gollo et al., 2014). That is, it may be the case that slower frequencies could be used to either inhibit or promote the relative contributions of different sensory observationscommunicated at faster gamma frequencies - to iterative rounds of Bayesian model selection. This kind of adaptive enhancement of prediction errors may help to reconcile predictive processing with findings that consciousness level and phenomenal binding have been associated with increases in gamma power and inter-electrode gamma coherence (Singer, 2001, 2007). Alternatively, it may merely be the case that more precise predictions tend to be accompanied by increased prediction errors, without observations being specifically enhanced through attentional selection mechanisms. In either case, predictive processing diverges with some more well-known ideas in suggesting that gamma-band activity may not itself generate consciousness, but instead indirectly modulates the updating of beliefs forming at slower frequencies.

\section{Towards new methods of estimating integrated information}

According to IWMT, IIT's maximal complexes and GNWT's workspaces are emergent eigenmodes of effective connectivity (Friston et al., 2014a; Atasoy et al., 2018). Considering the network properties of brains (Heuvel and Sporns, 2011), these self-organizing harmonic modes (SOHMs) are likely to be centered around deep portions of hierarchical generative models which may enable (via turbo-coding) convergence upon approximate posteriors (and empirical priors 
for subsequent rounds of Bayesian model selection). If this integrative view is accurate, then it may provide new means of evaluating phi estimation methods (Tegmark, 2016), with significance for both basic research and clinical practice. Reliable means of estimating phi are necessary for practical applications, as the formally-prescribed calculations are NP-hard (Mayner et al., 2018; Toker and Sommer, 2019), and so require simplifying assumptions for even modestly-sized causal networks. Nature, in contrast may implicitly perform such calculations nearly 'for free' via Hamilton's principle of least action. Unfortunately, Seth and colleagues (2019b) have found radically different integrated information estimates can be derived with seemingly reasonable modeling assumptions. If IWMT's proposal is correct- that phi corresponds to self-model-evidence; a claim which has recently received endorsement from Karl Friston (2020) - then applying different integration estimation methods to Bayesian networks may provide a ground truth for adjudicating between different estimation approaches.

If IWMT's proposal is correct in suggesting a complex with maximally irreducible causeeffect power (i.e., a MICS) is also a maximally informative subgraph, then this correspondence could provide further means of estimating integrated information (phi). Note: IWMT does not necessarily ascribe to the particular definition of phi provided by IIT 3.0, as a case could be made for integrated information being better reflected by the average/median degree of integration in an effective network-e.g. likely consequences of a random perturbation-rather than by its maximal irreducibility as indicated by a least damaging graph cut. According to FEPAI, neural dynamics can be viewed as implementing approximate Bayesian inference, where activation cascades constitute a message passing regime (Friston et al., 2017c; Parr et al., 2019b). Theoretically, differential rates of message passing may automatically discover maximally connected subnetworks (Mišić et al., 2015), and in doing so, converge on processes of variable elimination (Koller and Friedman, 2009). In variable elimination, marginal information from factors are progressively integrated into an induced graph — or maximal clique - thereby providing a maximally likely a posterior (MAP) estimate from the overall belief network. If maximal complexes tend to be centered on these maximal cliques (or are equivalent to them) these internally-directed cause-effect structures could have actual semantic content by virtue of containing (or entailing) MAP estimates over hidden causes that define self and world for embodied-embedded agents.

It is unclear whether these specific correspondences between probabilistic graphical modeling techniques and concepts from IIT will be found to be valid. However, if IWMT is accurate in claiming that self-cause-effect power in IIT corresponds with self-model-evidence in FEP-AI, then relative merits for different phi estimation techniques could be evaluated based on their abilities to track the inferential properties of Bayesian networks. Specifically, metrics of quality for phi estimates could be indicated by quicker convergence times for loopy message passing, more precise posterior distributions and accurate MAP estimates, and enhanced learning rate or inferential power more generally.

IWMT's synthesis could also potentially lead to novel phi estimation methods based on modeling processes by which complexes of integrated information emerge as self-organizing harmonic modes (SOHMs). Although a detailed handling is beyond the scope of the present discussion, useful means of estimating phi and modeling SOHM/complex formation may potentially be found in flow networks (cf. max-flow-min-cut theorem) (Dantzig and Fulkerson, 1955; Garg et al., 1996; Hoffman, 2003) and other kinds of physical systems. Further, game theoretically informed constructs such as Shapley centrality have been used in the study of dynamic networks (Chen and Teng, 2017), and such measures may be relevant for modeling 
processes by which complexes of cause-effect power emerge. Estimation techniques inspired by these kinds of analogies may potentially be more computationally tractable than other phi estimation methods, and may also provide further bridges between IIT and FEP-AI (and thereby GNWT when workspace dynamics are considered to represent Bayesian model selection).

Speculatively, it appears that there may be potentially fruitful correspondences between maximal complexes in IIT and the identification of the kernel (and/or nucleolus) of the core of a game (Schmeidler, 1969; Maschler et al., 1979; Maschler, 1992). But with respect to the core of a cooperative game, integrated information as cause-effect power would refer to a nexus of bargaining influence amongst players in a coalition. Here, I will provide an informal sketch of how such an analysis might proceed:

1. Persisting neuronal dynamics may be modeled as quasi-agents, with each pattern constituted by an implicit model for its continued existence (i.e., preserving their Markov blankets).

2. For each of these different quasi-agents, utility would be defined in terms of generating self-model-evidence, and cost would be defined in terms of prediction error-within a generalized Darwinian framework, these implicit utility functions would also be fitness functions (Safron, 2019b).

3. Hamilton's principle of least action implies that each dynamical pattern will always choose its best response for minimizing free energy (Kaila and Annila, 2008; Friston, 2019).

4. In this self-prediction game, stable coalitions (as cores) would correspond to equilibrium solutions, wherein competing and cooperating neuronal quasi-agents are not incentivized to leave the grand coalition, because doing so would increase free energy.

5. When stable game theoretic equilibria can be found, the center of gravity for these geometries also represent Shapley values, or solutions that balance the respective utilities and bargaining power for participating quasi-agents.

6. If the game being played entails hypotheses regarding latent causes, and if bargaining power is a function of predictive ability, then this Shapley value may also represent a precision-weighted probabilistic model of world states, or maximal (MAP) estimate derived thereof.

While admittedly speculative and underdeveloped, such a game theoretic derivation of complexes of integrated information - functioning as workspaces - would help provide a cybernetic grounding for IIT (and GNWT), answering the question of why there may be "something that it is like" to be a maximal complex, or workspace, or any physical system. Alternatively (and very speculatively), similar analyses (and implications) may potentially be derived by modeling neural dynamics as entailing a kind of prediction market (Conitzer, 2012), with estimated prices representing probabilities for the sufficient statistics for world models. These kinds of handlings of integrative processes may render notions of "neuronal coalitions" (Crick and Koch, 2003) as something more than a 'mere metaphor,' and could also give new meaning to Edelman's (2011b) description of consciousness as being realized by "dynamic cores" (Safron, 2019d).

\section{Beyond integrated information}

IIT has evolved as a theory over two decades of concerted effort, and further refinements and elaborations of the theory are currently being developed. This ongoing evolution has caused 
some people to question whether IIT's postulated mechanisms are truly grounded in axiomatic principles of phenomenology (Bayne, 2018), and whether its methods may contain questionable modeling assumptions. Indeed, many of the most practically useful (and highly face valid) phi estimation techniques rely on previous versions of the theory, such as estimating integrated information based on causal density (Barrett and Seth, 2011; Seth et al., 2011a).

Much skepticism regarding IIT has resulted from demonstrations of high phi values being associated with systems for which there are strong a priori reasons to suspect a lack of consciousness, such as the kinds of 2D grids used in expander graphs (Aaronson, 2014). Yet such objections to IIT's validity can be readily handled by considering integrated information to be necessary, but not sufficient for consciousness without the cybernetic grounding suggested by IWMT. However, the potential modeling capacity of even a single 2D grid should not be underestimated (Wang and Roychowdhury, 2019). With respect to the particular example of the dubious consciousness of expander graphs, it should be noted that such systems have many of the properties which may contribute to the computational power of brains, including small-world connectivity (Takagi, 2018), sparseness (Ahmad and Scheinkman, 2019), and ability to support error-correcting codes (Liu and Poulin, 2019). Theoretically, an arrangement of hierarchicallyorganized expander graphs could be used to implement predictive processing and may be functionally equivalent to the kinds of turbo coding adduced by IWMT. Nonetheless IWMT states that such systems will not be conscious unless their functionality enables coherentlyintegrated world modeling, which may be afforded in mammalian brains by posterior medial cortices with respect to visual phenomenology and a sense of quasi-Cartesian space.

Others have questioned the merit of emphasizing a single measure for the informational dynamics of complex systems (Mediano et al., 2019a). This work has challenged the assumption of pairwise causal interactions in networks, instead focusing on dynamical complexity in terms of the decomposition of integrated information into potentially coexisting modes of informational flows. These novel measures reveal that integration processes can be understood as aggregates of multiple heterogeneous phenomena such as informational storage, copy, transfer, erasure, downward causation, and upward causation. Promisingly, these decomposed measures of integrated information could allow for the creation of novel methods for assessing informational dynamics, which may be superior in some use cases.

IWMT agrees with Mediano et al. that integrated information is not the only valuable way to look at consciousness or complex systems more generally. Nonetheless, aggregations of heterogeneous phenomena can produce wholes that are greater than the sum of their parts. Mind and life are two such phenomena, and this kind of functional synergy may also apply to informational constructs (including mind and life). If integrated information corresponds to selfmodel-evidence as described by FEP-AI, then this would be a very special measure of dynamical complexity, potentially indicating the ability of whole systems to be both stable, adaptive, and even autonomous (Albantakis, 2017). Indeed, connections between integrated information and self-organized criticality further suggests that we may be dealing with a measure that applies to all systems capable of not just persisting, but evolving (Arsiwalla and Verschure, 2016; Arsiwalla et al., 2017; Hoffmann and Payton, 2018; Takagi, 2018).

\section{Conscious and unconscious cores and workspaces; physical substrates of agency}

Although a detailed handling is beyond the scope of the present discussion, k-core decomposition and percolation methods may be useful for estimating subnetworks (e.g. giant 
components) that are particularly likely to have high integrated information (Cellai et al., 2016; Bianconi, 2017; Kryven, 2019). An intriguing recent study used these kinds of techniques to track the transition from conscious to unconscious subliminal perceptual states (Arese Lucini et al., 2019). Somewhat surprisingly, the most connected kernel and inner core of the conscious state remained functionally active when the brain transitioned to the subliminal-state. Not only was the inner-most connectivity core of the brain potentially unconscious, but conscious access was lost by inactivating peripheral shells, suggesting the importance of sensorimotor information for enabling coherent experience. These findings suggest that consciousness may not be generated in the inner-most core, but at intermediate levels of hierarchical organization and abstraction (Prinz, 2017; Chang et al., 2019). This could be further interpreted as supporting the previously described hypothesis of perceptual binding via embodied-self models (ESMs), wherein action-oriented body maps may provide backbones for global workspaces.

These findings could also potentially illuminate otherwise mysterious phenomena, including things like intuitive cognition (Kalra et al., 2019), "tip-of-the tongue" effects, and even the roles of spontaneity in agency (Ismael, 2016; Hills, 2019). For example, some aspects of intuition and semi-conscious percepts may correspond to attractor dynamics accumulating in the (unconscious) inner-most core and outer shells and bypassing intermediate levels. If sufficiently robust, such processes may be capable of driving policy selection and conscious imaginings from networks supporting (consciously experienceable) embodied simulations-potentially the $1^{\text {st }}$ shell out from the inner core- but without sufficient robustness to be stably introspectable. Further, while agency might typically depend on predictability for the sake of controllability, there may be further ways in which limitations of self-prediction enhance overall control:

- Avoiding excessive exploitation (at the expense of exploration) in action selection (broadly construed to include mental acts with respect to attention and working memory).

- A process for generating novel possibilities as a source of counterfactuals for causal reasoning and planning.

- Game theoretic considerations such as undermining the ability of rival agents to plan agonistic strategies.

In these ways, somewhat paradoxically, agency may sometimes be enhanced by limiting the scope of volitional control.

Perhaps relatedly, recent work in artificial intelligence models the frontal pole as a recurrent neural network (RNN) whose capacity for bifurcation enables flexible action selection and predictive learning (Ha and Schmidhuber, 2018; Wang et al., 2018). RNN-like computational reservoirs have high potential for informational density due to the combinatorics of re-entrant connections, but feedforward hierarchies have the advantages of discrete compositionality (so affording precise control) and robustness-via-modularity (so affording separable optimization). Cortical systems may leverage both of these capacities by placing a recurrent bifurcating nexus on top of a more linear hierarchy of forward models over actionperception cycles (Tani, 2016). The capacity of RNNs to exert recursive causal influences on themselves means that they constitute chaotic systems with sensitivity to initial conditions. In these ways, the top of the cortical control hierarchy may be occupied by processes that are both consciously inaccessible and inherently uncontrollable. These deepest portions of cortex are also newest with respect to both evolution and development (LeDoux, 2019). It is also notable that these areas also appear to have most of the properties we normally associate with the essence of personhood (Hassabis et al., 2014), including individuality (Peña-Gómez et al., 2018; Toro-Serey et al., 2020), spontaneity (see above), and autonomy (James, 1879). 
If these models regarding the neural substrates of consciousness are accurate, then they may also help contextualize findings where agency appears to be missing, as famously described by the Libet (1983) experiment in which the subjective experience of motor commands were observed to come after highly predictive neural activity. Potential limitations of the paradigm notwithstanding (Dennett, 2003; Fifel, 2018; Maoz et al., 2019), the question arises as to how mental states could be causal, given that we expect causes to precede effects. Theoretically, people's reports regarding the feeling to act occurring after preparatory motor activity could be partially accounted for by effective connectivity with a largely unconscious inner core.

If this is the case, then is our sense of free will another grand illusion? Perhaps in some cases, but probably not with respect to the "varieties of free will worth wanting" (Dennett, 1981), as much meaningful executive control does not involve the spontaneous generation of motor deployment events. Indeed, such acts might primarily be governed by hierarchically lower levels, closer to primary modalities that have less access to richly connected networks whereby largescale (consciously accessible and controllable) coordinated activity would tend to be centered. Further, most actions do not occur as one-off events, but unfold within contexts that often involve a great deal of conscious imagining and planning, whose associated neural activity may drive much of overall dynamics. Similarly to the previously discussed case of the apparent flatness of consciousness and the supposed insubstantiality of selfhood, we may find ourselves denying the existence of "real patterns" (Dennett, 1991) based on investigations that were illequipped to capture the relevant phenomena. In some senses we might identify agency (and personhood) with overall systems with both conscious and unconscious components. Such systems (and persons) may not be strongly shaped by consciousness in any given moment, yet could be significantly consciously shaped over time. Agency may usually be more like the relationship between conductor and an orchestra, where conductors are neither omnipotent nor mere epiphenomena. Or to use the metaphor of the elephant and its rider: elephants with and without riders are very different "beast machines" (Seth and Tsakiris, 2018).

\section{Bayesian blur problems and solutions; Quantum consciousness? (no and yes)}

Before moving on to discuss how we may understand the qualities of particular kinds of experiences, we will now briefly visit the issue of the "Bayesian blur problem" (Clark, 2018). That is, how is it that a probabilistic world model can generate seemingly discrete perceptual experiences, rather than a superposition of possibilities? Functionally speaking, it may be desirable for the brain to provide discrete estimates of — or highly precise distributions overworld states for the sake of coherent action selection. However, a "Bayesian blur solution" could also be proposed, in that it may also be desirable to maintain full probability distributions with multiple possibilities kept in play for the sake of adaptation and exploration. In considering GNWT as implementing Bayesian model selection, it may be the case that brains obtain the best of both discrete and probabilistic modeling by dividing and conquering across different phases of cognitive cycles, or possibly across brain areas (Gazzaniga, 2018; McGilchrist, 2019). Global workspaces could allow for periods where multiple competing and cooperating hypotheses can remain in play, followed by winner-take-all dynamics when information is integrated and then broadcast.

Dehaene (2014) suggests that the formation of workspaces via ignition events can be understood as a kind of phase change akin to those observed in physical systems. Intriguingly, he goes onto propose that a potentially productive analogy could be found in models of wave 
function collapse in quantum physics, where a superposition of possibilities is reduced to a determinate classical world. IWMT considers this to be a promising avenue for future investigation. It may be similarly productive to explore whether multiple interpretations of quantum mechanics apply to varying degrees as abstract descriptions of varying information dynamics within minds, understood in terms of varieties of Bayesian model selection:

- Can entanglement be used to model changes in the precision of distribution as a result of coupling sub-systems?

- Do these interactions ever produce decoherence and collapse into discrete estimates of parameter values, including with causal roles for conscious access via frontal cortex (c.f. Copenhagen interpretation)?

- Could Bohmian mechanics be used to describe the functional significance of traveling waves or potential quantization of prediction errors in predictive processing?

- Could Everettian "many worlds" interpretations be used to describe branching hypothesis spaces during certain phases of cognitive cycles?

To be clear, these are all (very) highly speculative analogies for information dynamics, and quantum physical phenomena are likely not directly relevant to the brain's computational abilities in any meaningful sense, given the hot and crowded nature of biological systems (Tegmark, 2000). However, it may also be worth considering that brains may be able to emulate quantum computational principles via classical means (Borders et al., 2019; Coyle et al., 2019; Guillet et al., 2019).

\section{Neurophenomenology of IWMT: Qualia explained?}

We have reviewed some of the philosophical foundations, neural systems, and computational principles underlying IWMT. We will now expand these analyses to include considerations of prototypical qualia: the feeling of emotional experiences, including pleasure, pain, and desire. Each of these aspects of experience can be extremely rich in terms of their particular qualities, functionalities, and the ways these vary across contexts. Here, we will begin to explore how principles and mechanisms from FEP-AI can be used to begin to show how these experiences can be so fundamental and yet remain so mysterious.

\section{Emotions and Feelings}

In attempting to analyze the nature of emotional experience, perhaps some of the continuing mystery is due to a lack of agreement on terminology in affective neuroscience and psychology. Damasio et al. (2000, 2003, 2012, 2018), argue that emotions can be ascribed to the value-oriented behavior of all living organisms, including single-celled organisms such as bacteria. However, Damasio reserves the word "feeling" for the conscious re-representation of emotions. Feldman-Barrett and Joseph LeDoux (2017; 2019), in contrast, object to this more inclusive conceptualization of emotion, arguing instead that emotional language should be reserved for consciously experienced affective states that are expressed and constructed through interpretive processes. Indeed, LeDoux has even gone as far as to claim that emotions only arose via cultural evolution after the advent of language.

IWMT sees merit in both points of view. While less inclusive conceptualizations may avoid some kinds of confusions, they also miss the opportunity to identify ways in which value 
and integrated informational dynamics are essential to all life. IWMT takes an intermediate perspective, viewing emotions and feelings as the respective action and perception components of action-perception cycles over large-scale changes in organismic modes (Safron, 2019d). Relevant macroscale dynamics can include phenomena ranging from autonomic functions, to musculoskeletal body modifications (Sandrone and Safron, 2013), and also direct modifications of the nervous system via neuromodulatory systems and effective connectivity from neural areas with high effective centrality (e.g. the amygdala complex). In addition to this cybernetic formulation of emotions as a kind of action, and feelings as a kind of perception, IWMT further proposes that we add an additional distinction as to the extent to which the expression of emotions and sensing of feelings is of a conscious or unconscious variety. This use of terminology would support many of the rationales for the positions described above, as well as many folk intuitions and the ways that these are expressed in normal linguistic use. If useful for communicative clarity and ethical discussions, additional distinctions can be made as to whether consciousness involves basic phenomenal awareness or is of a more complex access or autonoetic variety.

LeDoux $(2016,2019)$ has argued that animals without complex language cannot be said to possess emotions, but merely have functional activity within "survival circuits." These claims are justified by language being required to provide syntactic structures for the construction of complex extended self-models; or as LeDoux states: "No self, no fear." This emphasis on the importance of selfhood is largely compatible with IWMT. Without extended self-processes, emotions and feelings will be qualitatively different than the kinds of emotions and feelings constructed by humans governed by (and governing) a symbolic order of being. However, IWMT's cybernetic formulation refers to the functional activity of survival circuits as entailing emotions, which may or may not be consciously expressed and felt. In this way, IWMT separates the idea of "something that it is like to be" from "something that it feels like" with respect to the nature of consciousness.

According to IWMT, all evolved cybernetic systems would have emotions and feelings, but only systems capable of coherent integrative world modeling would consciously experience those affects. These conscious systems likely include all mammals and birds, and possibly some reptiles or fish if they possess sufficiently elaborated palliums to be capable of hierarchical modeling (Ocaña et al., 2015; Suryanarayana et al., 2017), with large-scale integrative and information processing abilities further organized by the kinds of synchronous coherence and routing mechanisms enabled by the thalamic complex (Aru et al., 2019). Thus, IWMT takes a middle way between the perspectives described above in viewing emotions and feelings as ubiquitous features of life, while simultaneously recognizing qualitative differences that emerge when these phenomena are associated with consciousness of various kinds. Both more and less inclusive stances may be viewed as reasonable, but with respect to qualitatively different kinds of affective phenomena.

\section{What is value? Predictive coding models of reward prediction errors}

In the Free Energy Principle and Active Inference (FEP-AI) framework, minds and all other living systems can be described in terms of a single objective of self-model-evidence maximization and prediction error minimization. By this account, organisms begin development by implicitly predicting the rectification of homeostatic (and later reproductive) prediction errors, so forming a foundation out of which all subsequent models grow. With experience, these 
modeling efforts come to apply to the modeling processes themselves and the experiences they generate, including models of what is likely to cause changes in prediction error. That is, we come to predict ourselves minimizing prediction errors and experiencing associated mental states, including with respect to emotions and feelings. In this (both correlational and later causal) associative chaining story from early organismic experiences, organisms begin by being reinforced/punished by experiences accompanying homeostatic rectification. Over time, organisms come to learn reinforcement contingencies that make associated stimuli more or less likely to be available. Mechanistically, representations detecting these contingencies are themselves connected to midbrain dopamine-e.g. orbitofrontal cortex $\rightarrow$ accumbens shell $\rightarrow$ ventral tegmental area (Mannella et al., 2013) — so allowing cortical models to drive reinforcement/punishment signals, and so shape adaptive policies for action.

This account has parallels with work on meta-reinforcement learning from DeepMind (Wang et al., 2018), where systems are initially given primary reward functions that allow for learning secondary reward functions from experience. From an FEP-AI perspective, these secondary (and higher-order) cortical predictions would constitute meta-predictions for prediction error minimization. According to candidate trace models (Stolyarova, 2018), dopamine is likely to strengthen whatever predictions may have been most likely to contribute to its release by being most active leading up to phasic increases, so providing a partial solution to the credit assignment problem. If phasic dopamine increases are released proportionally to the rate of change of prediction error rectification (Schultz, 2010, 2016), then the more quickly something minimizes prediction error, the more it comes to be predicted.

In these ways, organisms would come to predict states of initial prediction error increases, so that these free energy gradients (experienced as desire, or "wanting") could be destroyed through enaction (experienced as pleasure, or "liking"). The creation and destruction of these gradients of anticipatory and consummatory reward would then stimulate dopamine release proportional to both the magnitude of prediction error reduction and the temporal interval at which it occurs. These mechanisms could shape organisms to predict themselves not only in homeostatic and reproductive states, but also diverging from these desirable states, to the degree that such discrepancies between goals and actualities are anticipated to be capable of being eliminated through actions.

\section{Curiosity and play/joy}

This prediction of prediction error minimization creates an interesting setup in which such organisms end up being surprised when they do not find themselves riding down steep enough gradients of prediction error (Joffily and Coricelli, 2013; Hesp et al., 2019). This is exactly what evolution would 'want' (Dawkins, 1996; Safron, 2019b), since there is no limit to how evolutionarily fit an organism can be, and so organisms ought to always seek opportunities for realizing value in new ways. Driving of dopamine release by reward prediction errors provides one means of realizing this evolutionary imperative for expansion in seeking opportunities for value realization. That is, if the mechanisms underlying reinforcement and behavioral disinhibition are only activated for unexpectedly good outcomes, then organisms will always find themselves seeking to explore the limits of what they can attain. This exploratory impulse may be even stronger if accompanied by opportunities for refining models and satisfying curiosity-based desires, so realizing the intrinsic value of learning in addition to the extrinsic 
value of utility maximization (Friston et al., 2017b; de Abril and Kanai, 2018; Gottlieb and Oudeyer, 2018; Koelsch et al., 2019).

Boredom, in contrast, would represent a punishing process that functions in an inverse fashion to curiosity (and play). One mechanism for implementing this negative incentive could be found in predictive coding via habituation. That is, if an organism comes to near perfectly predict rewards - or consider associated stimuli to be non-salient and so not orient attention to them - then this familiarity could result in prediction errors only being generated at lower levels of cortical hierarchies, which lack access to richly connected networks enabling widespread availability and consciousness (Safron, 2019d). This lack of prediction errors could result in reduced recognition of the features associated with those potential rewards. Both with respect to implicit predictions and explicit expectations, a previously rewarding event that does not fully register would be experienced as a let-down in contrast to expected value (Crespi, 1942; Cooper et al., 2009), so resulting in devaluation of stimuli and reduced probability of selecting associated policies. Almost paradoxically, by becoming less surprised by (or more familiar with) rewarding stimuli, an organism may end up becoming more surprised relative to reward anticipation, since the predicted reward never manifests, as it was never registered in the first place. Some evidence for this model can be found in over-rehearsed pleasurable acts being overly automatic, habitual, and progressively losing their hedonic tone. Between the twin masters of curiosity and boredom, such a setup would help shape agents that are always seeking to expand their repertoire of policies for value realization (to the extent these expansion efforts are expected to result in increasingly desirable outcomes).

Under FEP-AI, we ought to expect living organisms - by virtue of being successful at existing - to be equipped with (or constituted by) system-defining prior expectations (or preferences) in which they are optimizing models of themselves doing the kinds of things which would be required for survival, including foraging for information. This survival imperative requires organisms to enact system-world configurations dependent on policies with consequences in the future, and which also depend on policies not yet deployed. This means that successfully persisting adaptive systems must not only minimize free energy, but also expected free energy in (definitionally counterfactual) futures. These successful active inferential systems will expect themselves to be maximizing information gain (i.e., precision-weighted prediction errors), while also avoiding the accumulation of cybernetic entropy (Hirsh et al., 2012; Seth, 2014c; Dalege et al., 2018; Safron, 2019a, 2019d) in ways that would represent an existential threat to the system. Within FEP-AI, this dilemma of balancing stability/plasticity tradeoffs is (boundedly optimally) resolved by gradient descent over a singular objective functional of expected free energy.

The maximal rate of reduction in overall expected free energy will be found in situations where agents are able to simultaneously balance imperatives for maximizing both the intrinsic value of information/exploration, as well as the extrinsic value of realizing preferred world states. This situation may be referred to as play, or "PLAY" (Panksepp, 1998, 2007) —often subjectively experienced as "flow" states (Csikszentmihalyi, 1997)—which, in maximizing reward, represents an attracting state for an organism that places it precisely where it ought to be in order to maximize learning and evolutionary fitness (Kiverstein et al., 2019). That is, the balanced conditions of play attract agents to a zone of proximal development (Vasileva and Balyasnikova, 2019) — or "edge of the adjacent possible" (Kauffman and Clayton, 2006; Kauffman, 2014), and also the "edge of chaos" (Atasoy et al., 2019) — where learning rate is 
optimal, creating conditions that are neither overly or underly challenging for promoting skillful engagement with the world (Buchsbaum et al., 2012; Dhawale et al., 2019).

These considerations are also a large part of why we would not expect agents to minimize surprise by sequestering themselves in low-complexity environments, in addition to the fact that such conditions would increase homeostatic/reproductive prediction errors from the hypothalamic control column and the systems with which it (allostatically) couples. Such agents would both experience boredom and deprivation with respect to curiosity and play. Although we should also remember that this "Dark Room problem" (Friston et al., 2012c) is not completely solved by nature, as people often do seek out reduced complexity environments, whether due to the kinds of pathological beliefs associated with anxiety and depression, or by getting stuck at local maxima of excessive exploitation relative to exploration in model optimization.

\section{Synesthetic affects}

In the account describe above, all affect is ultimately associatively linked to the rectification of either homeostatic or reproductive error signals, for which interoceptive consequences may be some of the most reliable sources of information (Seth and Friston, 2016). However, these interoceptive signals have poor spatial localizability and controllability. If spatiotemporal and causal contextualization are necessary for enabling coherent experience, then these constraints on sense-making could result in interoceptive information being attributed to non-interoceptive sources. That is, the best available inference regarding these visceral (and vital) signals may be that they are caused by and inextricably part of the conditions with which they are associated. Theoretically, this could cause most interoception to take on a quasisynesthetic quality, wherein poorly localizable signals become intimately entangled with (or 'infused' into) more easily modeled proprioceptive and exteroceptive phenomena. For example, we may both feel our body from the inside, while also projecting these feelings onto and into associated objects. While it may seem odd to describe feelings as a kind of synesthesia, evidence continues to accumulate that all perception may have at least some degree of synesthetic phenomenology in terms of involving cross-modal blending (Fauconnier and Turner, 2003; Brang and Ramachandran, 2011; Sievers et al., 2013, 2019; Cuskley et al., 2019).

This synesthetic-affects hypothesis has some support from descriptions of pleasure as a kind of "gloss" applied to objects of hedonic experience (Berridge and Kringelbach, 2011). If accurate, this account could also explain part of why emotional experiences often have an ineffable quality when reported upon. That is, affects may heavily depend on information that is difficult to explicitly model, and for which modeling efforts usually involve a kind of anomalous inference that are personal feelings are inextricably — and essentially (Bloom, 2010) — part of the conditions that evoke them.

The synesthetic-affects hypothesis may not only explain some of the ways that our feelings 'color' the world — for both good and ill—but also the phenomenology of will with respect to both motivation and effort. Specifically, the feeling of willing may correspond to a hybrid percept in which interoceptive states are mapped onto the effector systems by which intentions are realized. In this way, in addition to helping to explain otherwise mysterious aspects of experience, synesthetic-affects may also have extensive functional consequences. Predictive coding accounts of emotional active inference have been proposed in which prediction errors from interoceptive states can be minimized through either a) changing autonomic conditions, or b) changing related world-states via mobilization of proprioceptive effector systems (Seth et al., 
2012; Barrett et al., 2016). Along these lines, if synesthetic phenomenology increases the extent to which interoceptive states are tightly coupled with actions and perceived outcomes, then this conjunction could help to establish affordance-informed salience mappings over perceptual contents, so facilitating action selection and planning (Farshidian et al., 2019). Mechanistically, these tight perceptual couplings could strengthen patterns of effective connectivity between interoceptive and proprioceptive modalities, so enabling greater control energy from networks whose dynamics are ultimately grounded in evolutionary fitness and histories of experiences with organismic value (Safron, 2019d). In theory, the subjective sense of realness (Seth, 2014b) for affective phenomena may substantially depend on relatively tight associations between emotions and outcomes, so contributing to synesthetic mappings between feelings and causes. If these linkages are broken - e.g. via insensitivity to interoceptive sensations or inabilities to imagine the realization of valued goals - the synesthetic infusions of interoceptive value into other percepts could be compromised, potentially contributing to clinical conditions like anhedonia, alexithymia, the negative symptoms of schizophrenia, and even Cotard's syndrome and Capgras illusions.

\section{The computational neurophenomenology of desire}

Dopamine plays important roles in FEP-AI (Friston et al., 2012b, 2014b; FitzGerald et al., 2015), as it modulates activity for representations of value-relevant stimuli, as well as the actions that tend to accompany those stimuli while pursuing desired outcomes. Functionally speaking, changing dopamine levels could be interpreted of as increasing the strength of the inference/prediction: "Things with value-relevant features are likely to be close in either space or time, or particularly relevant to what I think is happening or ought to be happening now." These features may include both external reward cues as well as multi-modal representations of activities involved in seeking valued goals, including minimizing the probability of undesirable outcomes.

In predictive processing accounts of goal-oriented behavior (Safron, 2019d), when an agent predicts itself obtaining valued outcomes, but has not yet realized these goals, it will generate prediction errors corresponding to discrepancies between representations for goal attainment, relative to estimated present states (potentially including imaginings of next likely states). These discrepancies may be generated from contrasting desired and present estimated states, potentially occurring at theta frequencies orchestrated by hippocampal-prefrontal coupling (Koster et al., 2018; Kunz et al., 2019). As these comparison operations proceed, discrepant features will be likely to increase their activity through predictive coding mechanisms, so drawing attention to and seeding imaginings with the most important features that need to be handled in some way, either by updating internal models or changing the world.

Much of the phenomenology of desire could potentially be explained as the prediction of attaining value, which then activates associated somatic and interoceptive concomitants of consummation, which are subjectively felt in body maps in those places most associated with value realization. If these sensations are accompanied by temporary net decreases in predicting the realization of homeostatic or reproductive value (Joffily and Coricelli, 2013; Hesp et al., 2019) — potentially mediated by opioid signaling (Leknes and Tracey, 2008; Berridge and Kringelbach, 2015) - this state would be likely to result in net negative affect accompanying these perceptions. In this way, the feeling of desire may be experienced as a kind of pain, with its particular characteristics depending on particular histories of associated experience. However, 
this experience of painful desire may quickly become pleasure if we then find ourselves predicting net increases in predicting the realization of value, so creating a pleasurable contrast with the discomfort of wanting. Further, as described above, the visceral concomitants of affective experience may then become entangled with exteroceptive and proprioceptive percepts in a quasi-synesthetic fashion.

To use a musical metaphor, in experiences of pain and/or/as unfulfilled desire, the overall melody will be played in a more minor - or entropic (Hirsh et al., 2012; Dalege et al., 2018) key/timbre. Alternatively, in experiences of pleasure and/or/as fulfilled desire, affective orchestras will play melodies with greater consonance. One could view such soundtracks to the (fully immersive virtual reality) movies of experience as a separate stream of information that helps to contextualize what is being seen on the screens over which we see stories unfold. However, it may be closer to experience to say that this metaphorical music enters into what we see and feel, imbuing (or coloring) it with meanings. Indeed, we may be able to find most of the principles of affective phenomena to be well-reflected in our experiences of music (Schopenhauer, 1844; Sacks, 2008; Koelsch et al., 2019), where we play with building and releasing tension, enjoying the rise and fall of more and less consonant (or less and more dissonant) melodies. In musical pleasure, we explore harmony and the contrast of disharmony, eventually expecting to return home to the wholeness of the tonic, but with the ability of our "experiencing selves" (Sayette et al., 2008; Kahneman, 2011; Zajchowski et al., 2017) to find satisfaction in the moment not necessarily being the reason that our "remembering selves" find ourselves attracted to particular songs.

The affective melodies played by neural orchestras will tend to be dominated by interoceptive modalities, the most ancient — both developmentally and evolutionarily speakingand reliable indicators of homeostatic and reproductive potential (Craig, 2009a; Porges, 2009; Damasio, 2012, 2018; Jennings et al., 2015). Do we have relaxed and dynamic cardiac rhythms? Is our breathing easy or forced? Do we feel warm-but not too hot —or cold? Are our bowels irritated or copacetic? Do we feel full or empty inside? Do we feel like our body is whole and strong, ours to command where we will, if we wanted it? Or do we feel damaged and weak? This interoceptive information speaks to foundations of life and the cores of value out of which persons may grow.

\section{Desiring to desire; transforming pain into pleasure and back again}

How can we reconcile the experience of desire as a species of pain in light of the fact that we often desire to desire? While desiring may sometimes be desirable, it is not a pleasant thing to be in a state of unsatisfied hunger or thirst without the belief that this situation will be manageable. To be hungry or thirsty without cessation is to predict moving away from homeostasis and survival. Unless an organism can be confident that it will eventually rectify this situation, failing to satisfy these desires reflects a true emergency situation. In this way, we would expect desire unsatisfied to be experienced as a kind of pain. But the pain of desire could then be transformed into pleasure - and back again (and so on) - by consummation, or the vivid imagination of attainment.

Can an agent ever come out ahead with this back and forth between pleasure and pain, either with respect its experiencing or remembering selves? How can motivation be maintained if all pleasures will ultimately be transformed back into kinds of pain? One explanation may potentially be found through predictive coding mechanisms, in that nervous systems will select 
for perception and memory that which changes the most, and so experiencing/remembering will likely to be dominated by the transitions between pleasure and pain, especially if accompanied by precipitous or punctuated alterations (Gopnik, 1998; Joffily and Coricelli, 2013; Safron, 2016; Hesp et al., 2019; Safron, 2019c). If consummation tends to rectify a situation more rapidly, and if seeking without finding results in a more gradual buildup of desire, then experience and memory for successfully enacted goals may have an overall pleasurable (and reinforcing) quality. Although of course this pleasure is not something that natural selection 'wanted' us to have and hold onto, but to be continually "SEEKING" (Panksepp, 1998), thereby maximizing evolutionary fitness.

\section{Why conscious affects?}

Consciously experienced affects may provide unified attractors for coordinating global organismic states (Craig, 2009a; Damasio, 2012). While affects may be unconscious, when made consciously accessible they allow for value-planning and continuous adjustments of actions. This mapping of hedonic states onto consciously introspectable models of enaction also provides a means of handling the credit assignment problem, via conjoining value and actions in both experience and memory. If affects took place "in the dark" without feeling like anything, they would be unable to strongly influence events, nor be coherently integrated into explicit modeling and planning, including plans involving pursuing those affects as ends in-and-of themselves, such as in the domains of play and art.

\section{Facing up to the meta-problem}

\section{A dialogue on the Hard problem of generative models}

IWMT suggests that principles from probability theory machine learning could be used to identify correspondences across multiple levels of analysis, where these bridging principles may be sufficiently powerful that we can claim to have made meaningful progress on explaining the nature of experience. While such an extraordinary claim may seem implausible, below I will offer two dialogues (Hofstadter, 1979, 2007; Hofstadter and Sander, 2013) on why the problem of explaining consciousness may appear to be Hard for some, but merely very (very!) difficult for others.

The Hard (maths) problem of generative models:

Mysterion: "Why is there something that it looks like to be the output layer of a generative model for images?"

Non-mysterion: "Because it was trained up on data with certain statistical regularities that can be reproduced when the model is inverted."

Mysterion: "No. I mean, how can those images be so rich in terms of color and texture and shape?"

Non-mysterion: "Because those features were part of the regularities of the world that the network was able to learn."

Mysterion: "But why should that look like anything?!"

Non-mysterion: "How much linear algebra do you know?" 
Mysterion: "Very little. Why?"

Non-mysterion: ...

Perhaps the Hard problem of consciousness may be felt to be solved (or unsolved) for similar reasons:

Mysterion: "Why should neural activity ever give rise to the qualities of experience? Why is there something that it is like?!"

Non-mysterion: "Because your nervous system is generating/filling-in a more complete sensorium from moment-to-moment based on inferences-to-the-best-guess from past experiences."

Mysterion: "But how can you ever explain that ineffable feeling of the experience of looking at the face of someone you know?"

Non-mysterion: "Because faces have been fundamental to your existence for your entire life, and the centrality of faces for your niche means that you're going to have many kinds of emotions/feelings/reactive-dispositions associated with them, and these are largely dependent on interoceptive sensations which are difficult to localize and tend to float free of their origins and get mapped onto other things in a quasi-synesthetic fashion, so much so that you consider this affect to be part of the essential nature of the things that tend to evoke those responses." Mysterion: "But why should that 'FEEL' like something?"

Non-mysterion: "Because generative models of interoceptive states are also invertible, with some of these inversions leading to actual changes in physiological interoceptive dynamics as part of homeostatic/allostatic regulation of self (and others) (i.e., emotions), which can themselves form the basis for further predictive modeling (i.e., feelings)." Mysterion: "But why should that feel like anything?!"

Non-mysterion: "How much systems and computational neuroscience do you know?" Mysterion: "Very little. Why?"

Non-mysterion: ...

\section{Additional solutions to the meta-problem}

At this point, at least two solutions to the meta-problem can be identified. The dialogue above attempts to illustrate how the availability of bridging principles may cause prospects for explaining consciousness to seem either impossible (perhaps even in principle), or merely (very (very!)) difficult. An additional solution to the meta-problem may be found in the nature of explanations for various qualia: while perhaps intuitive after consideration, at first glance some of the models proposed above seem to directly contradict experience, such as desire constituting a species of pain, and vice versa (Craig, 2003). Other explanations of aspects of experience may not necessarily contradict intuitions, yet may nonetheless seem irreducibly strange and so prima facie implausible, such as the model of synesthetic affects described above. However, if it is indeed the case that some of the most fundamental and familiar aspects of experience may also seem unrecognizable upon close inspection, then this is itself something in need of explanation.

Much of this seeming paradox of the unrecognizability of the most fundamental and familiar could be resolved if such aspects of experience are likely to become phenomenally transparent, and so resistant to introspection (Metzinger, 2010; Limanowski and Friston, 2018). Specifically, IWMT suggests that contents of perception in any given moment may be entailed 
by beta complexes as local zones of integration, but with these requiring further integration into larger complexes for phenomenal and access consciousness. Such broader integration may not end up occurring if predictive coding mechanisms are so successful that they are capable of "explaining away" aspects of experience via local beta complexes. Processes of iterative Bayesian model selection can occur at multiple levels of hierarchical depth, and so if explaining away observations is largely successful via beta complexes closer to the modalities, that information may never reach more richly-connected-cores/subnetworks capable of coherent world modeling and experienceable perception. In this way, that which is most fundamental and familiar may almost inevitably become nearly invisible to introspective access.

The meta-problem may be nearly as conceptually rich as the Hard problem itself. Further promising approaches may involve paradoxes from functional "Strange loops" and self-reference (Hofstadter, 2007), computational limits of recursion (Lloyd, 2012), and illusions deriving from the mechanisms by which egocentric perspective is established (Rudrauf et al., 2017). Finally, some solutions to the meta-problem may be sociological in nature, potentially reflecting a legacy of "physics envy" in the mind sciences (Lau and Michel, 2019). That is, not only did we lack bridging principles and understanding of embodiment as the core of selfhood and experience, but in many ways scientific practice both implicitly and explicitly denigrated subjectivity after the decline of introspectionism and the rise of behaviorism. Given this taboo on subjectivity-the very thing we would hope to explain to understand consciousness (Varela, 1996) — why should we have been surprised if it was difficult to make progress on the Hard problem, let alone the (meta) endeavor of seriously considering the nature of such attempts at understanding?

\section{Consciousness: here, there, but not everywhere}

\section{Selective pressures for complex cognition}

When did different forms of consciousness first evolve? This is an extremely difficult question to answer with any kind of confidence, as mental states do not leave fossils, but must be inferred from combining assumptions regarding the functional capacities of different information processing systems and their likely behavioral consequences. A broad range of selective pressures may have contributed to the advent of consciousness and further elaborations in conscious cognition as major transitions in evolution:

1. Cognitive arms races between predators and prey (Godfrey-Smith, 2016), where the evolution of jaws in fish may have been a particularly important milestone (Martik et al., 2019).

2. The transition of aquatic animals to land resulting in increased capacity for long-distance vision approximately 380 million years ago, and so increased adaptive significance for being able to plan ahead (MacIver et al., 2017; Mugan and MacIver, 2019).

3. Selection for precise visualization associated with reaching and grasping of food by prosimians with capable hands (Sagan, 1977).

4. Selection for cognition and visualization abilities to facilitate the coordination required for highly social animals (Tomasello, 2014), and perhaps especially pack-hunting species.

5. Selection for planning when standing afforded increased abilities to see ahead (Russon and Begun, 2007). Further selection for visualization may have occurred due to the 
challenges associated with bipedal running. Similar selective pressures for planning and predictive imagining may have also been encountered by flying animals in the context of collision avoidance (Emery, 2006).

6. Increased selection for precise visualizations with tool-use, including with respect to thrown projectiles during hunting. While such abilities are often considered to be separate from explicit cognition, there is also evidence that counterfactual imaginings are important for guiding implicit learning processes for complex motor sequences (Kilteni et al., 2018; MacKay, 2019).

\section{Cultural evolution of consciousness}

Further major advances in the evolution of consciousness may have been culturally mediated. Some of the primary advantages of developing higher order consciousness may have been its ability to facilitate social learning and social coordination. Indeed, intriguing work suggests that coordinating individuals may implement something akin to collaborative Bayesian inference via a precision weighted combination of communicated beliefs (Roepstorff and Frith, 2004; Bahrami et al., 2010, 2012; Frith, 2012; Bang et al., 2014). In terms of social coordination, it may be worth noting the common etymology of consciousness and conscience (Lewis, 1960; Frith and Metzinger, 2016), and the ways in which being able to intentionally control present and future actions - and also account for past actions - may have provided substantial multi-level selective pressures on elaborating conscious capacities.

One of the more extreme versions of socially constructed consciousness can be found in the work of Julian Jaynes (1976). Jaynes controversially proposed that ancient humans hallucinated voices, which acted as a kind of executive function (Sundberg, 2013), so allowing individuals to coordinate without complex reflexive self-consciousness. According to Jaynes, modern consciousness developed through a constructive process involving analogical extension from the kinds of particular embodied experiences that lend themselves towards inferring Cartesian theaters. He further speculates that there may have been a hemispheric division of labor with the voices being "spoken" from the right hemisphere and "heard" on the left via efferent copies. Occurring some time between the events of the Iliad and the Odyssey, the "breakdown of the bicameral mind" is proposed to be a crowding out of this hallucinatory way of being with an expansion of agency and cognitive complexity, stimulated by challenges to supporting sociocultural structures.

While many aspects of Jaynes' model could be questioned, it is clearly the case that selfconsciousness can take a variety of forms, and it may have also been the case that some of these conscious varieties could have co-evolved rapidly with sociocultural replicators (Damasio, 2012, 2018; Dennett, 2017; Henrich, 2017; LeDoux, 2019). It may have even been the case that symbolization has a stabilizing role on cognition and perception, and that without strong abstraction, we may have had a more permeable boundary between imagination and perception, perhaps verging on hallucinations, the threshold for which may be less than is commonly assumed (Sacks, 2013).

In Jaynes' constructed consciousness, he further distinguishes between a linguisticallyscaffolded "analogue I" and a "metaphorical me." IWMT recognizes this distinction between kinds of self-processes (James, 1890; Rochat, 2010), and also acknowledges that both kinds of selves could potentially be substantially elaborated via the power of analogical reasoning and metaphorical construction (Lakoff and Johnson, 1999; Gentner, 2010b; Safron, 2019a). 
However, IWMT also argues that much of Cartesian perspective may naturally derive from evolutionarily ancient systems enabling egocentric reference frames (Papez, J.W., 1937; Safron, 2019d).

\section{Against strong panpsychism}

Proponents of IIT have argued that consciousness is "what physics feels like from the inside" (Koch, 2012). This statement is consistent with IWMT and FEP-AI, since there is an important sense in which the generative modeling principles linking brains and minds are exactly the same as those linking all persisting systems with their dynamics. In this way, both IWMT and FEP-AI could be viewed as panprotopsychist (Friston et al., 2020) in that all persisting systems must exhibit certain mind-like qualities (i.e., intelligence) in order to temporarily resist the $2^{\text {nd }}$ law. However, IWMT is not panpsychist in that consciousness is restricted to those systems capable of coherently integrated world modeling. A relevant distinction may be found in FEP-AI with respect to the differences between "being a model" and "having a model" (Kirchhoff et al., 2018; Seth and Tsakiris, 2018; Ramstead et al., 2019):

- Being models: generative processes describing the evolution of organisms and their niches.

- Having models: subcomponents of organisms that model these generative processes. According to IWMT, experience would only emerge from systems that have models (Rocha, 2000; Gazzaniga, 2018); however, these models will be insufficient for consciousness without capacities to generate virtual worlds that are coherent with respect to space, time, and cause. Without these coherence-making properties, there would be nothing that it is like because there would be no world to speak of.

In brief, for there to be "something that it is like" (let alone something that it feels like), modeling processes must be capable of generating representations of coherent organism-centered lifeworlds, such that the time-evolving state transitions of the modeling system represent the inferential filling-in of a sensorium. According to IWMT, such models are unlikely to evolvedevelop if systems are incapable of supporting workspaces as dynamic cores of high degrees of integrated information, which must also be capable of producing integration and differentiation on timescales that are roughly proportional to the adaptive shaping of action-perception cycles whereby systems couple with the world, so enabling cybernetically-grounded meaning-making.

It has recently been suggested that cognition requires a functional organization in which agents are able to detach from the present moment and engage in counterfactual active inference (Corcoran et al., 2019). IWMT does not have a clear position on whether or not the word "cognition" ought to be reserved for systems capable of generating counterfactual simulations. However, IWMT does claim that counterfactual processing may be required for consciousness via the causal inferences it affords. Note: This claim is distinct from a recent proposal in which consciousness is viewed as necessarily emerging from the generation of counterfactual information (Kanai et al., 2019). Lacking causal reasoning abilities, a system will likely be incapable of generating a coherent (and so experienceable) world. However, it is unclear the extent of causal inference that is required in order to bring coherence to experience. Most simply, causation can be modeled in terms of a capacity for predicting state transitions, which may not require the full sophistication of causal analysis based on interventions and counterfactuals (Pearl and Mackenzie, 2018). However, more sophisticated inferential abilities are likely required to enable more advanced varieties of conscious capacities and experiences. 
Below we will now consider a variety of systems to clarify whether (or not) they could be said to possess consciousness from an IWMT perspective. All statements requiring the presence or absence of consciousness should be understood as both a) tentative and subject to revisions, and $b$ ) referring to basic phenomenal experience.

Are rocks conscious? (no)

No (Safron, 2020).

Are viruses conscious? (no)

No.

Are single-celled organisms conscious? (no)

No.

Are plants (or fungi?) conscious? (no (and no))

Functionally speaking, the primary 'purpose' of nervous systems is the adaptive shaping of behavior, where action-perception cycles have some correspondence with attracting states of the (proactively) reactive nexus. Plants move (e.g. growth; phototaxis; other tropisms), but these dynamics tend to unfold slowly, and so their reactive-proactive nexuses do not need to exhibit the fast dynamics afforded by neurons. Yet, the attracting states of plants may be considered to entail generative models of their extended phenotypes and niche-construction processes enacted with their environments. Plants may even have internal attractors whose dynamics come to represent their generative unfoldings, such that in addition to saying that these plant-environment systems are models in their very being/doing, it may also be the case that such plants have models that provide further levels of predictive control. However, it seems unlikely that such inner models can generate dynamics capable of affording the construction of an integrative coherent world model through adaptive active inference. Without such capacities, plants (and fungi) are likely to be unconscious forms of life.

Is C. elegans conscious? (probably not)

While the nervous system of $\mathrm{C}$. elegans possesses a rich club connectivity core (Towlson et al., 2013), the small size of these networks suggest that dynamics are likely to be enslaved by immediate contingencies of sensorimotor engagements, so preventing the kinds of counterfactual processing required for causal modeling, so preventing sufficient coherence for generating an experienceable world.

Are intelligent eusocial colonies conscious? (no)

Colonies have minds in that they must in some sense be (and possibly contain) models of the world. However, these hive minds likely lack consciousness, with one of the primary reasons being the lack of sufficiently powerful constraints/affordances provided by embodiments with 
well-organized effector and sensory systems. Without such organization, eusocial insect colonies will be unable to generate tight conjunctions between action and perception, will be unable to afford detection and controllability of sensorimotor contingencies, and will be unable to infer a coherent world centered on a lived body. This is not to deny the potential power of swarm intelligence. Some colonies may even engage in some kinds of self-reflexive modeling with proto-metacognition (e.g. precision-weighted implicit beliefs); however, this modeling would likely still be of an unconscious variety.

\section{Are insects conscious? (maybe some (or all?) of them)}

Insects can have highly complex brains with 100's of thousands of neurons. Insect brains may even have homologous structures to those found in vertebrate nervous systems, such as the mushroom bodies as functionally similar to the striatum (Barnstedt et al., 2016; Bello-Medina et al., 2016). Even more, the insect central complex may not only enable rich club organization, but also bases for spatial mapping and navigation (Pfeiffer and Homberg, 2014; Honkanen et al., 2019), potentially organized according to egocentric reference frames (Fisher et al., 2019; Kim et al., 2019), so providing possible functional analogues with mammalian systems (Papez, J.W., 1937; Dillingham et al., 2015; Wijesinghe et al., 2015; Chaudhuri et al., 2019). However, unless these systems are capable of supporting inner dynamics that are divorced from the immediacy of sensorimotor engagements, they will likely be unable to engage in the counterfactual and causal reasoning required for coherent world modeling.

Are brainstems conscious? (probably not)

Panskepp, Damasio, and Solms (Panksepp, 2005; Damasio, 2012; Solms and Friston, 2018) have all argued for a subcortical basis for feeling awareness, drawing on evidence from emotional expressions in anencephalic patients and the preservation of feelings with bilateral insula damage (Damasio et al., 2013). As described above, IWMT agrees that these core affects can be described using the language of emotions and feelings. However, these affects are likely only made conscious via re-representation by processes capable of coherent world modeling, likely depending on either a thalamocortical system or functionally similar homologue (DugasFord et al., 2012; Shanahan, 2012). With respect to the particular evidence cited in defense of a conscious brainstem, the following counter-arguments are suggested:

1. We do not know what anencephalic patients are experiencing.

2. Although interoceptive inference may primarily center on the insular hierarchy, cingulate cortex may be able to engage in some degree of perceptual inference of interoceptive states, since all cortex has both sensory and motor properties (Hawkins and Blakeslee, 2004).

3. Many aspects of feelings may not be purely interoceptive, but may also involve proprioceptive and exteroceptive dynamics, such as body-posture modificationsperhaps especially with respect to the face and hands-with particular organismic and ecological significances.

4. Interoceptive information may also be substantially instantiated in non-insular (and noncingulate) body maps in adult brains; over the course of development, predictive coding mechanisms are likely to cause interoceptive representations to become more broadly (and sparsely/robustly) distributed with time and experience. 
5. Hierarchical organization can be observed within the colliculi and more broadly down into the spine, with the parabrachical nucleus and nucleus tractus solitarus providing integration over multiple kinds of bodily information; however, these structures are qualitatively different from the thalamocortical system, and are unlikely to be able to hierarchically model a world in which particular entities can be situated relative to other entities with specific attributes, coherently organized with respect to space, time, and cause for system and world.

The consciousness of anencephalic patients is even more unlikely in light of the absence of functioning entorhinal/hippocampal systems and the spatiotemporal organization of experience so afforded. One could offer a counter argument that patients with severe medial temporal lobe damage have preserved consciousness. However, this argument is not particularly convincing for somewhat similar reasons to those involved with respect to the insula-damage findings discussed above. That is, insular cortices may be developmentally necessary for the emergence of conscious feelings, and the entorhinal/hippocampal system may be (developmentally) necessary for the generation of any consciousness whatsoever. Consciousness may be preserved with damage to this core system for memory and imagination in adulthood (Hassabis and Maguire, 2009), but it is unlikely that coherent world models could be developed with severely disrupted capacities for remembering and counterfactual processing (and thereby causal reasoning).

Are reptiles and fish conscious? (maybe some (or all (or none)) of them)

It is unclear whether some (or all (or any)) reptiles and fish are conscious. Both phyla have cortical homologues capable of supporting body maps (Ocaña et al., 2015; Suryanarayana et al., 2017). However, it is unclear whether there are any reptile or fish species where palliums possess sufficient hierarchical complexity for coherent world modeling. It may be the case that basic phenomenal consciousness is sufficiently enabled by state transitions between autoencoded body maps being organized according to spatiotemporal trajectories from the entorhinal/hippocampal system. In theory, the sequential generation of body states could be accompanied by phenomenality, even without being contextualized within coherent world models. Such body mapping would be insufficient for establishing either counterfactual processing or a flexible point of view on the world, but these generative processes may nonetheless be accompanied by some kind of experience. This would push the advent of consciousness as far back as 560 million years ago with our common ancestors with lampreys (Suryanarayana et al., 2020). Further, in line with the previously mentioned theory of vision being expanded by extra-aquatic existence (so affording/requiring more advanced planning), it may be the case that reptiles - having made the transition to land - may have evolved palliums capable of entailing more sophisticated generative models over sensorimotor states. IWMT remains agnostic on these issues.

Are octopi (and squids (and cuttlefish)) conscious? (maybe (possibly (I hope not...)))

"The most merciful thing in the world, I think, is the inability of the human mind to correlate all its contents. We live on a placid island of ignorance in the midst of black seas of infinity, and it was not meant that we should voyage far. The sciences, each straining in its own direction, have hitherto harmed us little; but some day the piecing together of dissociated knowledge will open up such terrifying vistas of reality, and of our frightful position therein, that we shall either go mad from the revelation or flee from the light into the peace and safety of a new dark age." 
The intelligence of cephalopods has received much attention in recent years (GodfreySmith, 2016). Most of the neurons in cephalopods are distributed across their multiple arms, with their brains appearing to serve a coordinating function. Whether such coordination efforts involve integrative world modeling is unclear. High degrees of intelligence and the complexity of controlling multiple arms may suggest elaborate modeling capacities, and so consciousness. However, it should be remembered that intelligent behavior can sometimes be achieved through simple means (Taylor et al., 2012; Lind, 2018; Vasas and Chittka, 2019), and it should also be remembered that much of motor control may be simplified via cleverly designed body plans (Pfeifer and Bongard, 2006).

Are the arms of conscious cephalopods conscious? (no)

No.

Are newborn infants conscious? (probably (maybe?))

Analysis of the brain activity of infants suggest that integrated information increases over the course of development, with major advances occurring between 35 and 40 weeks of age (Isler et al., 2018). While the intelligence of infants may be grossly underestimated (Gopnik, 2009), it is unclear when infants actually acquire a capacity for coherent world modeling. Some evidence for consciousness in infants is provided by the observation of waveforms that have some of the characteristics of P300 ERP responses and global ignition (Dehaene, 2014). If IWMT is accurate, then we may find ourselves in an ethically complicated position where infants acquire basic phenomenal consciousness at some indeterminate time after birth. However, IWMT may also help to shed light on some other ethical quandaries, such as the moral status of brain organoids. That is, brain organoids may only become conscious after they are able to recapitulate the kinds of inferential dynamics afforded by the mammalian brain (and avian homologues), including structures such as those participating in the Papez circuit and egocentric reference frames it affords; e.g. thalamic and posterior cingulate integration of head position, via vestibular information from the inner ears and stretch-receptor information from the neck (Papez, J.W., 1937; Wijesinghe et al., 2015; Chaudhuri et al., 2019), so providing a coherent point of view on the world. (Note: This juxtaposition of infants and cerebral organoids is both unfortunate and awkward, although that is often the way with ethical quandaries.)

Will artificial general intelligence be conscious? (probably)

What is intelligence? Intelligence has been defined in terms of the ability to achieve goals across a broad range of environments (Legg and Hutter, 2006, 2007). IWMT and FEP-AI would both argue that this is an accurate view, but where goal attainment fundamentally depends on predictive abilities (cf. Good regulator theorem) (Conant and Ashby, 1970). That is, intelligence is nothing more than modeling ability, given resource constraints. IWMT suggests that developmental robotics may be the most promising route for creating strong AI. In biological systems, the foundational task of controlling bodies makes it such that minds and brains may be organized around representations of bodies (Safron, 2019d), so connecting affordances to 
perceptions, so enabling efficient action selection prioritized based on use-potential, and so solving the "frame problem" of determining which information is more or less likely to be relevant in a given situation (Shanahan and Baars, 2005; Dennett, 2018; Linson et al., 2018).

IWMT further proposes that the enduring problems of AI may be unsolvable without machine consciousness. While intelligence and consciousness may be separable in theory, they may not be practically separable due to the requirements of dealing with the otherwise unsurmountable challenges of handling combinatorial explosions from under-constrained inference spaces (Safron, 2019a, 2019d). However, conscious embodied agents restrict their search of hypothesis spaces based on similarity to past experiences, which are fruitfully constrained based on realizable affordances. Consciousness further provides flexibly accessible mappings of system-world configurations, both of which may be required for the more general deployment of artificially intelligent systems, perhaps especially in domains such as robotics. Natural language understanding may depend on consciousness to an even greater extent, as it is becoming increasingly apparent that robust linguistic competence requires an extensive base of common ground - and common sense (literally speaking) - in order to handle the ambiguities associated with speech and text (Marcus and Davis, 2019; Mitchell, 2019).

IIT, in contrast, argues that artificially intelligent systems could recapitulate all of the functional properties of human minds - including full brain emulations - yet still lack consciousness if they are run on architectures using serial processing operations (Tononi and Koch, 2015). This is a place where IWMT is more inclusive than IIT. It is unclear why integrated information analyses can only be applied to the causal relations of physical systems. Rather, IWMT argues that cause-effect power may be maximal for temporally extended processes, including those involving virtual machines. While the potential consciousness of AI is a deeply important matter, this particular issue may be practically moot in that human+ levels of general intelligence may require massively parallel 'neuromorphic' implementations.

\section{A tentative timeline for the evolution-development of consciousness according to IWMT}

- Being a model where dynamics entail implicitly predictive modeling processes (everything that exists; 13.45 billion years old within this universe); almost certainly nothing that it is like.

- Being a model that has a model in the form of complex inner states that track engagements with the world in an adaptive (i.e., predictive) fashion, but without centralized integration structures (all life; 3.7 billion years old); probably nothing that it is like.

- Being a model that has a model with centralized integration structures (e.g. nervous systems), but not ones capable of generating coherent world models (all nervous systems; $>1$ billion years old); proto-awareness; probably nothing that it is like.

- Being a model that has a model with sub-models that generate sensorimotor states for the organism's embodiment, but is incapable of coherently modeling causal dependencies between system-world relations (all vertebrates; 560 million years old); proto-creature consciousness; unclear whether or not there is anything that it is like.

- Being a model that has a model that has sub-models capable of producing phenomenal coherence (all animals with well-developed hierarchical memory systems; 430-200 million years old); basic phenomenality and beginnings of consciousness proper; something that it is like. 
- Being a model that has a model that has sub-models capable of modeling phenomenally coherent sub-models (humans and possibly some other non-human animals; 1-2 million years old); higher order consciousness; introspectable something that it is like, and where this accessibility and likeness qualitatively changes the nature of subjectivity.

- Being a nested modeling process capable of generating counterfactually rich causal models with respect to both explicit subjectively experienced and intersubjectively entrained modeling efforts (all humans; 70,000-40,000 years old?); ancestral/child consciousness and the beginning of agency proper; capable of imagining what it might be like under counterfactual possibilities.

- Being a nested modeling process capable of supporting self-processes in an entrainment relation with (sharable) diachronic narratives and explicit recursive self-reference, including with respect to cultural contexts ( 40,000-3,000 years old?); modern self/otherconsciousness as an evolving generative process capable of creating/constructing an enormous variety of meta-aware intentionally-shaped experiences; many things that it is like and could be like.

- Being an even more elaborate set of intersecting (and sometimes nested) modeling processes (possible future artificial intelligences, and possibly some advanced alien species (if they exist)); unclear what it is/will-be like.

\section{Conclusion}

In revisiting IWMT we have discussed topics ranging from first philosophy to the future of intelligence. Opinions will surely differ as to whether we have arrived at a satisfying solution to the Hard problem of consciousness (and the meta-issue as to whether this is even a real problem). Regardless of whether the Hard problem can be felt to be (dis)solved, we have only begun to explore the nature of conscious experiences.

Some directions for future work:

- What are the different forms and enabling mechanisms for self-consciousness?

- To what degree are different forms of consciousness constructed via social pressures on evolutionary and developmental timescales?

- What questions are we not asking right now, but ought to be?

\section{Acknowledgements}

I would like to thank all my family, friends, and mentors for their patience and support during the many years in which I have been developing these ideas.

I would like to thank Karl Friston for his intellectual mentorship, his encouragement with moving forward, and for giving me a never-ending source of inspiration.

I would like to thank Giulio Tononi, Bernard Baars, Stanislas Dehaene, Anil Seth, and Andy Clark for helping to inspire this synthesis. 
I would like to thank Amelia Thomley and Victoria Klimaj for their companionship, Maxwell Ramstead for his friendship (and for helping me to see the extent to which FEP-AI realizes the potential of both generalized evolution and intersubjectivity), Casper Hesp for his friendship (and for helping me to understand the potential of active inference and the nature of valence), Mike Johnson for his friendship and for introducing me to connectome harmonics (and helping me to see both the power of formalism and the world as music), Colin DeYoung for his friendship and endlessly fascinating conversations, Brook Ziporyn for waking me from my dogmatic slumbers (and helping me see the power of flexible-but-grounded perspectivism), Marcia Grabowecky and Ken Paller for being both true teachers and friends, Mike Bailey for his friendship and for teaching me how to do science, Heather Hoffmann for being Heather, and many (many) others.

I would also like to thank John Sylla for his friendship, and for introducing me to his concept of consciousness as an Adaptive Standing Wave (ASW), which is highly similar to my proposal of modeling synchronizing dynamics in terms of self-organizing harmonic modes (SOHMs). The term SOHMs was chosen to emphasize potential connections with the Harmonic Brain Modes framework (thanks to Selen Atasoy and her collaborators). In some ways, ASWs may be a superior name for SOHMs, in that capacity for adaptation applies not only to the processes by which meta-stable synchronized ensembles (temporarily) form, but to their capacity for ongoing evolution via self-organized criticality. The adaptive capacity of ASWs/SOHMs also provides further bridges to the Free Energy Principle and Active Inference (FEP-AI) framework, as well as theories centering on neuronal coalitions, integrated information, dynamic cores, and global workspaces, in that all of these conceptualizations describe dynamic complexes of integration, which can (and should) also be viewed in terms of generalized Darwinism. Indeed, FEP-AI suggests that to the extent harmonics can be identified on some level of granularity, then to that degree we may be dealing with systems that can be formally understood as (necessarily) always evolving.

I would like to thank Nick Hay for his friendship, and for our ongoing conversation/exploration about the nature of intelligence and for the ways it might be realized in biological and artificial systems. May he help to create truly intelligent machines and all the potential that could be so realized.

We shall not cease from exploration And the end of all our exploring

Will be to arrive where we started And know the place for the first time. -T.S. Eliot, "Little Gidding" 


\section{References}

Aaronson, S. (2014). Shtetl-Optimized » Blog Archive» Why I Am Not An Integrated Information Theorist (or, The Unconscious Expander). Available at: https://www.scottaaronson.com/blog/?p=1799 [Accessed December $11,2019]$.

Abid, G. (2019). Deflating inflation: the connection (or lack thereof) between decisional and metacognitive processes and visual phenomenology. Neurosci. Conscious. 2019. doi:10.1093/nc/niz015.

Adams, R., Shipp, S., and Friston, K. J. (2013). Predictions not commands: active inference in the motor system. Brain Struct. Funct. 218, 611-643. doi:10.1007/s00429-012-0475-5.

Ahmad, S., and Scheinkman, L. (2019). How Can We Be So Dense? The Benefits of Using Highly Sparse Representations. ArXiv Prepr. ArXiv190311257.

Ainslie, G. (2005). Précis of Breakdown of Will. Behav. Brain Sci. 28, 635-650; discussion 650-673. doi:10.1017/S0140525X05000117.

Ainslie, G. (2010). Picoeconomics: The Strategic Interaction of Successive Motivational States within the Person. Reissue edition. Cambridge: Cambridge University Press.

Albantakis, L. (2017). A Tale of Two Animats: What does it take to have goals? ArXiv170510854 Cs Q-Bio. Available at: http://arxiv.org/abs/1705.10854 [Accessed June 13, 2019].

Arese Lucini, F., Del Ferraro, G., Sigman, M., and Makse, H. A. (2019). How the Brain Transitions from Conscious to Subliminal Perception. Neuroscience 411, 280-290. doi:10.1016/j.neuroscience.2019.03.047.

Arsiwalla, X. D., Mediano, P. A. M., and Verschure, P. F. M. J. (2017). Spectral Modes of Network Dynamics Reveal Increased Informational Complexity Near Criticality. ArXiv170701446 Phys. Q-Bio. Available at: http://arxiv.org/abs/1707.01446 [Accessed June 16, 2019].

Arsiwalla, X. D., and Verschure, P. F. M. J. (2016). High Integrated Information in Complex Networks Near Criticality. in Artificial Neural Networks and Machine Learning - ICANN 2016 Lecture Notes in Computer Science., eds. A. E. P. Villa, P. Masulli, and A. J. Pons Rivero (Springer International Publishing), 184191.

Aru, J., Suzuki, M., Rutiku, R., Larkum, M. E., and Bachmann, T. (2019). Coupling the State and Contents of Consciousness. Front. Syst. Neurosci. 13. doi:10.3389/fnsys.2019.00043.

Atasoy, S., Deco, G., and Kringelbach, M. L. (2019). "Playing at the Edge of Criticality: Expanded Whole-Brain Repertoire of Connectome-Harmonics," in The Functional Role of Critical Dynamics in Neural Systems Springer Series on Bio- and Neurosystems., eds. N. Tomen, J. M. Herrmann, and U. Ernst (Cham: Springer International Publishing), 27-45. doi:10.1007/978-3-030-20965-0_2.

Atasoy, S., Deco, G., Kringelbach, M. L., and Pearson, J. (2018). Harmonic Brain Modes: A Unifying Framework for Linking Space and Time in Brain Dynamics. Neurosci. Rev. J. Bringing Neurobiol. Neurol. Psychiatry 24, 277-293. doi:10.1177/1073858417728032.

Bahrami, B., Olsen, K., Bang, D., Roepstorff, A., Rees, G., and Frith, C. (2012). Together, slowly but surely: the role of social interaction and feedback on the build-up of benefit in collective decision-making. J. Exp. Psychol. Hum. Percept. Perform. 38, 3-8. doi:10.1037/a0025708.

Bahrami, B., Olsen, K., Latham, P. E., Roepstorff, A., Rees, G., and Frith, C. D. (2010). Optimally interacting minds. Science 329, 1081-1085. doi:10.1126/science.1185718.

Baldassano, C., Chen, J., Zadbood, A., Pillow, J. W., Hasson, U., and Norman, K. A. (2017). Discovering Event Structure in Continuous Narrative Perception and Memory. Neuron 95, 709-721.e5. doi:10.1016/j.neuron.2017.06.041.

Bang, D., Fusaroli, R., Tylén, K., Olsen, K., Latham, P. E., Lau, J. Y. F., et al. (2014). Does interaction matter? Testing whether a confidence heuristic can replace interaction in collective decision-making. Conscious. Cogn. 26, 13-23. doi:10.1016/j.concog.2014.02.002.

Baram, A. B., Muller, T. H., Nili, H., Garvert, M., and Behrens, T. E. J. (2019). Entorhinal and ventromedial prefrontal cortices abstract and generalise the structure of reinforcement learning problems. bioRxiv, 827253. doi:10.1101/827253.

Barnstedt, O., Owald, D., Felsenberg, J., Brain, R., Moszynski, J.-P., Talbot, C. B., et al. (2016). Memory-Relevant Mushroom Body Output Synapses Are Cholinergic. Neuron 89, 1237-1247. doi:10.1016/j.neuron.2016.02.015.

Barrett, A. B., and Seth, A. K. (2011). Practical Measures of Integrated Information for Time-Series Data. PLOS Comput. Biol. 7, e1001052. doi:10.1371/journal.pcbi.1001052.

Barrett, L. F. (2017). How Emotions Are Made: The Secret Life of the Brain. Houghton Mifflin Harcourt. 
Barrett, L. F., Quigley, K. S., and Hamilton, P. (2016). An active inference theory of allostasis and interoception in depression. Philos. Trans. R. Soc. Lond. B. Biol. Sci. 371. doi:10.1098/rstb.2016.0011.

Barsalou, L. W. (2009). Simulation, situated conceptualization, and prediction. Philos. Trans. R. Soc. B Biol. Sci. 364, 1281-1289. doi:10.1098/rstb.2008.0319.

Barsalou, L. W. (2010). Grounded cognition: past, present, and future. Top. Cogn. Sci. 2, 716-724. doi:10.1111/j.1756-8765.2010.01115.x.

Bartolomei, F., and Naccache, L. (2011). The global workspace (GW) theory of consciousness and epilepsy. Behav. Neurol. 24, 67-74. doi:10.3233/BEN-2011-0313.

Bastos, A. M., Usrey, W. M., Adams, R. A., Mangun, G. R., Fries, P., and Friston, K. J. (2012). Canonical microcircuits for predictive coding. Neuron 76, 695-711. doi:10.1016/j.neuron.2012.10.038.

Battiston Federico, Guillon Jeremy, Chavez Mario, Latora Vito, and De Vico Fallani Fabrizio (2018). Multiplex core-periphery organization of the human connectome. J. R. Soc. Interface 15, 20180514. doi:10.1098/rsif.2018.0514.

Bayne, T. (2018). On the axiomatic foundations of the integrated information theory of consciousness. Neurosci. Conscious. 2018, niy007. doi:10.1093/nc/niy007.

Bellmund, J. L., Deuker, L., Navarro Schröder, T., and Doeller, C. F. (2016). Grid-cell representations in mental simulation. eLife 5, e17089. doi:10.7554/eLife.17089.

Bellmund, J. L. S., Gärdenfors, P., Moser, E. I., and Doeller, C. F. (2018). Navigating cognition: Spatial codes for human thinking. Science 362, eaat6766. doi:10.1126/science.aat6766.

Bello-Medina, P. C., Flores, G., Quirarte, G. L., McGaugh, J. L., and Alcalá, R. A. P. (2016). Mushroom spine dynamics in medium spiny neurons of dorsal striatum associated with memory of moderate and intense training. Proc. Natl. Acad. Sci. 113, E6516-E6525. doi:10.1073/pnas.1613680113.

Bergouignan, L., Nyberg, L., and Ehrsson, H. H. (2014). Out-of-body-induced hippocampal amnesia. Proc. Natl. Acad. Sci. 111, 4421-4426. doi:10.1073/pnas.1318801111.

Berridge, K. C., and Kringelbach, M. L. (2011). Building a neuroscience of pleasure and well-being. Psychol. WellBeing 1, 1-3. doi:10.1186/2211-1522-1-3.

Berridge, K. C., and Kringelbach, M. L. (2015). Pleasure systems in the brain. Neuron 86, 646-664. doi:10.1016/j.neuron.2015.02.018.

Berrou, C., and Glavieux, A. (1996). Near optimum error correcting coding and decoding: turbo-codes. IEEE Trans. Commun. 44, 1261-1271. doi:10.1109/26.539767.

Berrou, C., Glavieux, A., and Thitimajshima, P. (1993). Near Shannon limit error-correcting coding and decoding: Turbo-codes. 1. in Proceedings of ICC '93 - IEEE International Conference on Communications, 10641070 vol.2. doi:10.1109/ICC.1993.397441.

Betzel, R. F., Fukushima, M., He, Y., Zuo, X.-N., and Sporns, O. (2016). Dynamic fluctuations coincide with periods of high and low modularity in resting-state functional brain networks. NeuroImage 127, 287-297. doi:10.1016/j.neuroimage.2015.12.001.

Bianconi, G. (2017). Fluctuations in percolation of sparse complex networks. Phys. Rev. E 96, 012302. doi:10.1103/PhysRevE.96.012302.

Blakeslee, S., and Blakeslee, M. (2008). The Body Has a Mind of Its Own: How Body Maps in Your Brain Help You Do (Almost) Everything Better. Random House Publishing Group.

Bloom, P. (2010). How Pleasure Works: The New Science of Why We Like What We Like. 1ST ed. W. W. Norton \& Company.

Boly, M., Massimini, M., Tsuchiya, N., Postle, B. R., Koch, C., and Tononi, G. (2017). Are the Neural Correlates of Consciousness in the Front or in the Back of the Cerebral Cortex? Clinical and Neuroimaging Evidence. $J$. Neurosci. 37, 9603-9613. doi:10.1523/JNEUROSCI.3218-16.2017.

Bor, D., Schwartzman, D. J., Barrett, A. B., and Seth, A. K. (2017). Theta-burst transcranial magnetic stimulation to the prefrontal or parietal cortex does not impair metacognitive visual awareness. PLOS ONE 12, e0171793. doi:10.1371/journal.pone.0171793.

Borders, W. A., Pervaiz, A. Z., Fukami, S., Camsari, K. Y., Ohno, H., and Datta, S. (2019). Integer factorization using stochastic magnetic tunnel junctions. Nature 573, 390-393. doi:10.1038/s41586-019-1557-9.

Brang, D., and Ramachandran, V. S. (2011). Survival of the Synesthesia Gene: Why Do People Hear Colors and Taste Words? PLOS Biol. 9, e1001205. doi:10.1371/journal.pbio.1001205.

Brown, R., Lau, H., and LeDoux, J. E. (2019). Understanding the Higher-Order Approach to Consciousness. Trends Cogn. Sci. 23, 754-768. doi:10.1016/j.tics.2019.06.009. 
Buchsbaum, D., Bridgers, S., Skolnick Weisberg, D., and Gopnik, A. (2012). The power of possibility: causal learning, counterfactual reasoning, and pretend play. Philos. Trans. R. Soc. B Biol. Sci. 367, $2202-2212$. doi:10.1098/rstb.2012.0122.

Buckner, R. L., and Krienen, F. M. (2013). The evolution of distributed association networks in the human brain. Trends Cogn. Sci. 17, 648-665. doi:10.1016/j.tics.2013.09.017.

Buonomano, D. (2017). Your Brain Is a Time Machine: The Neuroscience and Physics of Time. W. W. Norton \& Company.

Butterfield, J. (2014). Reduction, Emergence and Renormalization. ArXiv14064354 Phys. Physicsquant-Ph. Available at: http://arxiv.org/abs/1406.4354 [Accessed June 13, 2019].

Buzsáki, G., and Tingley, D. (2018). Space and Time: The Hippocampus as a Sequence Generator. Trends Cogn. Sci. 22, 853-869. doi:10.1016/j.tics.2018.07.006.

Buzsáki, G., and Watson, B. O. (2012). Brain rhythms and neural syntax: implications for efficient coding of cognitive content and neuropsychiatric disease. Dialogues Clin. Neurosci. 14, 345-367.

Canolty, R. T., Ganguly, K., Kennerley, S. W., Cadieu, C. F., Koepsell, K., Wallis, J. D., et al. (2010). Oscillatory phase coupling coordinates anatomically dispersed functional cell assemblies. Proc. Natl. Acad. Sci. U.S. A. 107, 17356-17361. doi:10.1073/pnas.1008306107.

Canolty, R. T., and Knight, R. T. (2010). The functional role of cross-frequency coupling. Trends Cogn. Sci. 14, 506-515. doi:10.1016/j.tics.2010.09.001.

Cardinali, L., Frassinetti, F., Brozzoli, C., Urquizar, C., Roy, A. C., and Farnè, A. (2009). Tool-use induces morphological updating of the body schema. Curr. Biol. 19, R478-R479. doi:10.1016/j.cub.2009.05.009.

Carhart-Harris, R. L. (2018). The entropic brain - revisited. Neuropharmacology 142, 167-178. doi:10.1016/j.neuropharm.2018.03.010.

Carhart-Harris, R. L., and Friston, K. J. (2010). The default-mode, ego-functions and free-energy: a neurobiological account of Freudian ideas. Brain J. Neurol. 133, 1265-1283. doi:10.1093/brain/awq010.

Cellai, D., Dorogovtsev, S. N., and Bianconi, G. (2016). Message passing theory for percolation models on multiplex networks with link overlap. Phys. Rev. E 94, 032301. doi:10.1103/PhysRevE.94.032301.

Chalmers, D. J. (1995). Facing Up to the Problem of Consciousness. J. Conscious. Stud. 2, 200-19.

Chalmers, D. J. (2018). The Meta-Problem of Consciousness. J. Conscious. Stud. 25. Available at: https://www.ingentaconnect.com/content/imp/jcs/2018/00000025/f0020009/art00001.

Chang, A. Y. C., Biehl, M., Yu, Y., and Kanai, R. (2019). Information Closure Theory of Consciousness. ArXiv190913045 Q-Bio. Available at: http://arxiv.org/abs/1909.13045 [Accessed October 26, 2019 ].

Chater, N. (2018). Mind Is Flat: The Remarkable Shallowness of the Improvising Brain. Yale University Press.

Chaudhuri, R., Gerçek, B., Pandey, B., Peyrache, A., and Fiete, I. (2019). The intrinsic attractor manifold and population dynamics of a canonical cognitive circuit across waking and sleep. Nat. Neurosci. 22, 15121520. doi:10.1038/s41593-019-0460-x.

Chen, W., and Teng, S.-H. (2017). Interplay between Social Influence and Network Centrality: A Comparative Study on Shapley Centrality and Single-Node-Influence Centrality. ArXiv160203780 Phys. Available at: http://arxiv.org/abs/1602.03780 [Accessed December 11, 2019].

Chernyak, N., Kang, C., and Kushnir, T. (2019). The cultural roots of free will beliefs: How Singaporean and U.S. Children judge and explain possibilities for action in interpersonal contexts. Dev. Psychol. 55, 866-876. doi:10.1037/dev0000670.

Clark, A. (2018). Beyond the "Bayesian Blur": Predictive Processing and the Nature of Subjective Experience. $J$. Conscious. Stud. 25, 71-87.

Conant, R., C., and Ashby, W. R. (1970). Every good regulator of a system must be a model of that system. Int. J. Syst. Sci. 1, 89-97. doi:10.1080/00207727008920220.

Conitzer, V. (2012). Prediction Markets, Mechanism Design, and Cooperative Game Theory. ArXiv12052654 Cs. Available at: http://arxiv.org/abs/1205.2654 [Accessed January 29, 2020].

Cooper, J. C., Hollon, N. G., Wimmer, G. E., and Knutson, B. (2009). Available alternative incentives modulate anticipatory nucleus accumbens activation. Soc. Cogn. Affect. Neurosci. 4, 409-416. doi:10.1093/scan/nsp031.

Corcoran, A. W., Pezzulo, G., and Hohwy, J. (2019). From Allostatic Agents to Counterfactual Cognisers: Active Inference, Biological Regulation, and The Origins of Cognition. doi:10.20944/preprints201911.0083.v1.

Coupé, C., Oh, Y. M., Dediu, D., and Pellegrino, F. (2019). Different languages, similar encoding efficiency: Comparable information rates across the human communicative niche. Sci. Adv. 5, eaaw2594. doi:10.1126/sciadv.aaw2594. 
Coyle, B., Mills, D., Danos, V., and Kashefi, E. (2019). The Born Supremacy: Quantum Advantage and Training of an Ising Born Machine. ArXiv190402214 Quant-Ph. Available at: http://arxiv.org/abs/1904.02214 [Accessed June 16, 2019].

Craig, A. D. (2003). A new view of pain as a homeostatic emotion. Trends Neurosci. 26, 303-307. doi:10.1016/s0166-2236(03)00123-1.

Craig, A. D. B. (2009a). How do you feel--now? The anterior insula and human awareness. Nat. Rev. Neurosci. 10, 59-70. doi:10.1038/nrn2555.

Craig, A. D. (Bud) (2009b). Emotional moments across time: a possible neural basis for time perception in the anterior insula. Philos. Trans. R. Soc. B Biol. Sci. 364, 1933-1942. doi:10.1098/rstb.2009.0008.

Crespi, L. P. (1942). Quantitative Variation of Incentive and Performance in the White Rat. Am. J. Psychol. 55, 467517. doi:10.2307/1417120.

Crick, F., and Koch, C. (2003). A framework for consciousness. Nat. Neurosci. 6, 119-126. doi:10.1038/nn0203119.

Csikszentmihalyi, M. (1997). Finding Flow: The Psychology of Engagement with Everyday Life. Basic Books.

Cuskley, C., Dingemanse, M., Kirby, S., and van Leeuwen, T. M. (2019). Cross-modal associations and synesthesia: Categorical perception and structure in vowel-color mappings in a large online sample. Behav. Res. Methods 51, 1651-1675. doi:10.3758/s13428-019-01203-7.

Dalege, J., Borsboom, D., Harreveld, F. van, and Maas, H. L. J. van der (2018). The Attitudinal Entropy (AE) Framework as a General Theory of Individual Attitudes. Psychol. Inq. 29, 175-193. doi:10.1080/1047840X.2018.1537246.

Damasio, A. (2000). The Feeling of What Happens: Body and Emotion in the Making of Consciousness. 1st ed. Mariner Books.

Damasio, A. (2003). Looking for Spinoza: Joy, Sorrow, and the Feeling Brain. 1st ed. Houghton Mifflin Harcourt.

Damasio, A. (2012). Self Comes to Mind: Constructing the Conscious Brain. Reprint edition. New York: Vintage.

Damasio, A., Damasio, H., and Tranel, D. (2013). Persistence of Feelings and Sentience after Bilateral Damage of the Insula. Cereb. Cortex N. Y. NY 23, 833-846. doi:10.1093/cercor/bhs077.

Damasio, A. R. (2018). The Strange Order of Things: Life, Feeling, and the Making of Cultures. Pantheon Books.

Dantzig, G. B., and Fulkerson, D. R. (1955). On the Max Flow Min Cut Theorem of Networks. Available at: https://www.rand.org/pubs/papers/P826.html [Accessed June 13, 2019].

Davey, C. G., and Harrison, B. J. (2018). The brain's center of gravity: how the default mode network helps us to understand the self. World Psychiatry 17, 278-279. doi:10.1002/wps.20553.

Dawkins, R. (1996). River Out Of Eden: A Darwinian View Of Life. Basic Books.

Dayan, P., Hinton, G. E., Neal, R. M., and Zemel, R. S. (1995). The Helmholtz machine. Neural Comput. 7, 889904.

de Abril, I. M., and Kanai, R. (2018). A unified strategy for implementing curiosity and empowerment driven reinforcement learning. ArXiv180606505 Cs. Available at: http://arxiv.org/abs/1806.06505 [Accessed December 15, 2018].

De Kock, L. (2016). Helmholtz's Kant revisited (Once more). The all-pervasive nature of Helmholtz's struggle with Kant's Anschauung. Stud. Hist. Philos. Sci. 56, 20-32. doi:10.1016/j.shpsa.2015.10.009.

Deco, G., Cruzat, J., Cabral, J., Tagliazucchi, E., Laufs, H., Logothetis, N. K., et al. (2019). Awakening: Predicting external stimulation to force transitions between different brain states. Proc. Natl. Acad. Sci. 116, 1808818097. doi:10.1073/pnas.1905534116.

Deco, G., and Kringelbach, M. L. (2016). Metastability and Coherence: Extending the Communication through Coherence Hypothesis Using A Whole-Brain Computational Perspective. Trends Neurosci. 39, 125-135. doi:10.1016/j.tins.2016.01.001.

Dehaene, S. (2014). Consciousness and the Brain: Deciphering How the Brain Codes Our Thoughts. New York, New York: Viking.

Dennett, D. (1981). Brainstorms: Philosophical Essays on Mind and Psychology. Cambridge, Mass.: The MIT Press.

Dennett, D. (1991). Real Patterns. J. Philos. 88, 27-51. doi:10.2307/2027085.

Dennett, D. (1992). Consciousness Explained. 1st ed. Back Bay Books.

Dennett, D. (2003). Freedom Evolves. illustrated edition. Viking Adult.

Dennett, D. (2017). From Bacteria to Bach and Back: The Evolution of Minds. 1 edition. New York: W. W. Norton \& Company.

Dennett, D. C. (2014). "The self as the center of narrative gravity," in Self and consciousness (Psychology Press), $111-123$. 
Dennett, D. C. (2018). Facing up to the hard question of consciousness. Philos. Trans. R. Soc. B Biol. Sci. 373. doi:10.1098/rstb.2017.0342.

Dhawale, A. K., Miyamoto, Y. R., Smith, M. A., and Ölveczky, B. P. (2019). Adaptive Regulation of Motor Variability. Curr. Biol. 29, 3551-3562.e7. doi:10.1016/j.cub.2019.08.052.

Dillingham, C. M., Frizzati, A., Nelson, A. J. D., and Vann, S. D. (2015). How do mammillary body inputs contribute to anterior thalamic function? Neurosci. Biobehav. Rev. 54, 108-119. doi:10.1016/j.neubiorev.2014.07.025.

Doersch, C. (2016). Tutorial on Variational Autoencoders. ArXiv160605908 Cs Stat. Available at: http://arxiv.org/abs/1606.05908 [Accessed March 27, 2020].

Drew, P. J., Winder, A. T., and Zhang, Q. (2019). Twitches, Blinks, and Fidgets: Important Generators of Ongoing Neural Activity. The Neuroscientist 25, 298-313. doi:10.1177/1073858418805427.

D’Souza, R. M., and Nagler, J. (2015). Anomalous critical and supercritical phenomena in explosive percolation. Nat. Phys. 11, 531-538. doi:10.1038/nphys3378.

Dugas-Ford, J., Rowell, J. J., and Ragsdale, C. W. (2012). Cell-type homologies and the origins of the neocortex. Proc. Natl. Acad. Sci. 109, 16974-16979. doi:10.1073/pnas.1204773109.

Edelman, G. (2001). Consciousness: The Remembered Present. Ann. N. Y. Acad. Sci. 929, 111-122. doi:10.1111/j.1749-6632.2001.tb05711.x.

Edelman, G., Gally, J. A., and Baars, B. J. (2011a). Biology of consciousness. Front. Psychol. $2,4$. doi:10.3389/fpsyg.2011.00004.

Edelman, G., Gally, J. A., and Baars, B. J. (2011b). Biology of consciousness. Front. Psychol. 2, 4. doi:10.3389/fpsyg.2011.00004.

Edelman, G. J. (1987). Neural Darwinism: The Theory Of Neuronal Group Selection. First Edition. Basic Books.

Eguchi, A., Horii, T., Nagai, T., Kanai, R., and Oizumi, M. (2020). An Information Theoretic Approach to Reveal the Formation of Shared Representations. Front. Comput. Neurosci. 14. doi:10.3389/fncom.2020.00001.

Elton, M. (2000). Consciouness: Only at the personal level. Philos. Explor. 3, 25-42. doi:10.1080/13869790008520979.

Emery, N. J. (2006). Cognitive ornithology: the evolution of avian intelligence. Philos. Trans. R. Soc. B Biol. Sci. 361, 23-43. doi:10.1098/rstb.2005.1736.

Farshidian, F., Hoeller, D., and Hutter, M. (2019). Deep Value Model Predictive Control. ArXiv191003358 Cs Stat. Available at: http://arxiv.org/abs/1910.03358 [Accessed January 29, 2020].

Fauconnier, G., and Turner, M. (2003). The Way We Think: Conceptual Blending And The Mind's Hidden Complexities. Reprint edition. New York, NY: Basic Books.

Faul, L., St. Jacques, P. L., DeRosa, J. T., Parikh, N., and De Brigard, F. (2020). Differential contribution of anterior and posterior midline regions during mental simulation of counterfactual and perspective shifts in autobiographical memories. NeuroImage 215, 116843. doi:10.1016/j.neuroimage.2020.116843.

Fifel, K. (2018). Readiness Potential and Neuronal Determinism: New Insights on Libet Experiment. J. Neurosci. 38, 784-786. doi:10.1523/JNEUROSCI.3136-17.2017.

Fisher, Y. E., Lu, J., D’Alessandro, I., and Wilson, R. I. (2019). Sensorimotor experience remaps visual input to a heading-direction network. Nature 576, 121-125. doi:10.1038/s41586-019-1772-4.

FitzGerald, T. H. B., Dolan, R. J., and Friston, K. J. (2015). Dopamine, reward learning, and active inference. Front. Comput. Neurosci. 9. doi:10.3389/fncom.2015.00136.

Fraccaro, M., Kamronn, S., Paquet, U., and Winther, O. (2017). A disentangled recognition and nonlinear dynamics model for unsupervised learning. in Advances in Neural Information Processing Systems, 3601-3610.

Fries, P. (2015). Rhythms For Cognition: Communication Through Coherence. Neuron 88, 220 -235. doi:10.1016/j.neuron.2015.09.034.

Friston, K. J. (2017). Self-evidencing babies: Commentary on "Mentalizing homeostasis: The social origins of interoceptive inference" by Fotopoulou \& Tsakiris. Neuropsychoanalysis 19, 43-47.

Friston, K. J. (2018). Am I Self-Conscious? (Or Does Self-Organization Entail Self-Consciousness?). Front. Psychol. 9. doi:10.3389/fpsyg.2018.00579.

Friston, K. J. (2019). A free energy principle for a particular physics. ArXiv190610184 Q-Bio. Available at: http://arxiv.org/abs/1906.10184 [Accessed July 1, 2019].

Friston, K. J., Breakspear, M., and Deco, G. (2012a). Perception and self-organized instability. Front. Comput. Neurosci. 6. doi:10.3389/fncom.2012.00044.

Friston, K. J., FitzGerald, T., Rigoli, F., Schwartenbeck, P., and Pezzulo, G. (2017a). Active Inference: A Process Theory. Neural Comput. 29, 1-49. doi:10.1162/NECO_a_00912. 
Friston, K. J., Kahan, J., Razi, A., Stephan, K. E., and Sporns, O. (2014a). On nodes and modes in resting state fMRI. NeuroImage 99, 533-547. doi:10.1016/j.neuroimage.2014.05.056.

Friston, K. J., Lin, M., Frith, C. D., Pezzulo, G., Hobson, J. A., and Ondobaka, S. (2017b). Active Inference, Curiosity and Insight. Neural Comput. 29, 2633-2683. doi:10.1162/neco_a_00999.

Friston, K. J., Parr, T., and de Vries, B. (2017c). The graphical brain: Belief propagation and active inference. Netw. Neurosci. 1, 381-414. doi:10.1162/NETN_a_00018.

Friston, K. J., Schwartenbeck, P., FitzGerald, T., Moutoussis, M., Behrens, T., and Dolan, R. J. (2014b). The anatomy of choice: dopamine and decision-making. Philos. Trans. R. Soc. B Biol. Sci. 369. doi:10.1098/rstb.2013.0481.

Friston, K. J., Shiner, T., FitzGerald, T., Galea, J. M., Adams, R., Brown, H., et al. (2012b). Dopamine, affordance and active inference. PLoS Comput. Biol. 8, e1002327. doi:10.1371/journal.pcbi.1002327.

Friston, K. J., Thornton, C., and Clark, A. (2012c). Free-Energy Minimization and the Dark-Room Problem. Front. Psychol. 3. doi:10.3389/fpsyg.2012.00130.

Friston, K. J., Wiese, W., and Hobson, J. A. (2020). Sentience and the Origins of Consciousness: From Cartesian Duality to Markovian Monism. Entropy 22, 516. doi:10.3390/e22050516.

Frith, C. D. (2012). The role of metacognition in human social interactions. Philos. Trans. R. Soc. Lond. B. Biol. Sci. 367, 2213-2223. doi:10.1098/rstb.2012.0123.

Frith, C. D., and Metzinger, T. K. (2016). 12 What's the Use of Consciousness ? How the Stab of Conscience Made Us Really Conscious. in doi:10.7551/mitpress/10709.003.0014.

Fujita, K., Carnevale, J. J., and Trope, Y. (2018). Understanding Self-Control as a Whole vs. Part Dynamic. Neuroethics 11, 283-296. doi:10.1007/s12152-016-9250-2.

Gao, Z., Davis, C., Thomas, A. M., Economo, M. N., Abrego, A. M., Svoboda, K., et al. (2018). A cortico-cerebellar loop for motor planning. Nature 563, 113-116. doi:10.1038/s41586-018-0633-x.

Garg, N., Vazirani, V. V., and Yannakakis, M. (1996). Approximate max-flow min-(multi) cut theorems and their applications. SIAM J. Comput. 25, 235-251.

Gazzaniga, M. S. (2018). The Consciousness Instinct: Unraveling the Mystery of How the Brain Makes the Mind. Farrar, Straus and Giroux.

Gentner, D. (2010a). Bootstrapping the Mind: Analogical Processes and Symbol Systems. Cogn. Sci. 34, 752-775. doi:10.1111/j.1551-6709.2010.01114.x.

Gentner, D. (2010b). Bootstrapping the Mind: Analogical Processes and Symbol Systems. Cogn. Sci. 34, 752-775. doi:10.1111/j.1551-6709.2010.01114.x.

Gershman, S. J. (2019). The Generative Adversarial Brain. Front. Artif. Intell. 2. doi:10.3389/frai.2019.00018.

Gibson, J. J. (1977). "The theory of affordances," in Perceiving, Acting, and Knowing. Towards an Ecological Psychology. Hoboken, NJ: John Wiley \&amp; Sons Inc.

Godfrey-Smith, P. (2016). Other Minds: The Octopus, the Sea, and the Deep Origins of Consciousness. Farrar, Straus and Giroux.

Gollo, L. L., Mirasso, C., Sporns, O., and Breakspear, M. (2014). Mechanisms of zero-lag synchronization in cortical motifs. PLoS Comput. Biol. 10, e1003548. doi:10.1371/journal.pcbi.1003548.

Gopnik, A. (1998). Explanation as Orgasm*. Minds Mach. 8, 101-118. doi:10.1023/A:1008290415597.

Gopnik, A. (2009). The Philosophical Baby: What Children's Minds Tell Us About Truth, Love, and the Meaning of Life. Macmillan.

Gottlieb, J., and Oudeyer, P.-Y. (2018). Towards a neuroscience of active sampling and curiosity. Nat. Rev. Neurosci. 19, 758-770. doi:10.1038/s41583-018-0078-0.

Graves, A., Wayne, G., and Danihelka, I. (2014). Neural turing machines. ArXiv Prepr. ArXiv14105401.

Graziano, M. S. A. (2013). Consciousness and the Social Brain. Oxford University Press.

Graziano, M. S. A. (2018a). The Spaces Between Us: A Story of Neuroscience, Evolution, and Human Nature. Oxford University Press.

Graziano, M. S. A. (2018b). The temporoparietal junction and awareness. Neurosci. Conscious. 2018. doi:10.1093/nc/niy005.

Graziano, M. S. A. (2019). Rethinking consciousness: a scientific theory of subjective experience. First Edition. New York: WWNorton \& Company.

Grossberg, S. (2017). Towards solving the hard problem of consciousness: The varieties of brain resonances and the conscious experiences that they support. Neural Netw. 87, 38-95. doi:10.1016/j.neunet.2016.11.003.

Guillet, S., Roget, M., Arrighi, P., and Molfetta, G. D. (2019). The Grover search as a naturally occurring phenomenon. ArXiv abs/1908.11213. 
Guterstam, A., Kean, H. H., Webb, T. W., Kean, F. S., and Graziano, M. S. A. (2019). Implicit model of other people's visual attention as an invisible, force-carrying beam projecting from the eyes. Proc. Natl. Acad. Sci. 116, 328-333. doi:10.1073/pnas.1816581115.

Ha, D., and Schmidhuber, J. (2018). World Models. ArXiv180310122 Cs Stat. doi:10.5281/zenodo.1207631.

Haken, H. (1977). Synergetics. Phys. Bull. 28, 412.

Harari, Y. N. (2015). Sapiens: A Brief History of Humankind. 1st edition. New York: Harper.

Hassabis, D., Kumaran, D., Summerfield, C., and Botvinick, M. (2017). Neuroscience-Inspired Artificial Intelligence. Neuron 95, 245-258. doi:10.1016/j.neuron.2017.06.011.

Hassabis, D., and Maguire, E. A. (2009). The construction system of the brain. Philos. Trans. R. Soc. Lond. B. Biol. Sci. 364, 1263-1271. doi:10.1098/rstb.2008.0296.

Hassabis, D., Spreng, R. N., Rusu, A. A., Robbins, C. A., Mar, R. A., and Schacter, D. L. (2014). Imagine All the People: How the Brain Creates and Uses Personality Models to Predict Behavior. Cereb. Cortex 24, 19791987. doi:10.1093/cercor/bht042.

Haun, A., and Tononi, G. (2019). Why Does Space Feel the Way it Does? Towards a Principled Account of Spatial Experience. Entropy 21, 1160. doi:10.3390/e21121160.

Hawkins, J., and Blakeslee, S. (2004). On Intelligence. Adapted. Times Books.

Hayek, F. A. (1952). The Sensory Order: An Inquiry into the Foundations of Theoretical Psychology. University Of Chicago Press.

Heeger, D. J. (2017). Theory of cortical function. Proc. Natl. Acad. Sci. U. S. A. 114, 1773-1782. doi:10.1073/pnas.1619788114.

Helmholtz, H. (1878). "The Facts in Perception," in Selected Writings of Hermann Helmholtz, ed. R. Kahl (Wesleyan University Press).

Henrich, J. (2017). The Secret of Our Success: How Culture Is Driving Human Evolution, Domesticating Our Species, and Making Us Smarter. Princeton University Press.

Herzog, M. H., Kammer, T., and Scharnowski, F. (2016). Time Slices: What Is the Duration of a Percept? PLOS Biol. 14, e1002433. doi:10.1371/journal.pbio.1002433.

Hesp, C., Smith, R., Allen, M., Friston, K., and Ramstead, M. (2019). Deeply Felt Affect: The Emergence of Valence in Deep Active Inference. PsyArXiv doi:10.31234/osf.io/62pfd.

Heuvel, M. P. van den, Kahn, R. S., Goñi, J., and Sporns, O. (2012). High-cost, high-capacity backbone for global brain communication. Proc. Natl. Acad. Sci. 109, 11372-11377. doi:10.1073/pnas.1203593109.

Heuvel, M. P. van den, and Sporns, O. (2011). Rich-Club Organization of the Human Connectome. J. Neurosci. 31, 15775-15786. doi:10.1523/JNEUROSCI.3539-11.2011.

Hills, T. T. (2019). Neurocognitive free will. Proc. Biol. Sci. 286, 20190510. doi:10.1098/rspb.2019.0510.

Hinton, G. How to do backpropagation in a brain. 22.

Hirsh, J. B., Mar, R. A., and Peterson, J. B. (2012). Psychological entropy: a framework for understanding uncertainty-related anxiety. Psychol. Rev. 119, 304-320. doi:10.1037/a0026767.

Hirsh, J. B., Mar, R. A., and Peterson, J. B. (2013). Personal narratives as the highest level of cognitive integration. Behav. Brain Sci. 36, 216-217. doi:10.1017/S0140525X12002269.

Hoffman, A. J. (2003). “A generalization of max flow-min cut," in Selected Papers Of Alan J Hoffman: With Commentary (World Scientific), 275-282.

Hoffmann, H., and Payton, D. W. (2018). Optimization by Self-Organized Criticality. Sci. Rep. 8, 2358. doi:10.1038/s41598-018-20275-7.

Hofstadter, D. R. (1979). Gödel, Escher, Bach: An Eternal Golden Braid. 20 Anv. Basic Books.

Hofstadter, D. R. (2007). I Am a Strange Loop. Basic Books.

Hofstadter, D., and Sander, E. (2013). Surfaces and Essences: Analogy as the Fuel and Fire of Thinking. 1 edition. New York: Basic Books.

Honkanen, A., Adden, A., Freitas, J. da S., and Heinze, S. (2019). The insect central complex and the neural basis of navigational strategies. J. Exp. Biol. 222. doi:10.1242/jeb.188854.

Hout, M. C., Papesh, M. H., and Goldinger, S. D. (2013). Multidimensional scaling. Wiley Interdiscip. Rev. Cogn. Sci. 4, 93-103. doi:10.1002/wcs.1203.

Hu, F., Kamigaki, T., Zhang, Z., Zhang, S., Dan, U., and Dan, Y. (2019). Prefrontal Corticotectal Neurons Enhance Visual Processing through the Superior Colliculus and Pulvinar Thalamus. Neuron 0. doi:10.1016/j.neuron.2019.09.019.

Hume, D. (1993). An Enquiry Concerning Human Understanding: with Hume's Abstract of A Treatise of Human Nature and A Letter from a Gentleman to His Friend in Edinburgh. Second Edition,2 edition., ed. E. Steinberg Indianapolis: Hackett Publishing Company, Inc. 
Imaizumi, S., and Tanno, Y. (2019). Intentional binding coincides with explicit sense of agency. Conscious. Cogn. 67, 1-15. doi:10.1016/j.concog.2018.11.005.

Isler, J. R., Stark, R. I., Grieve, P. G., Welch, M. G., and Myers, M. M. (2018). Integrated information in the EEG of preterm infants increases with family nurture intervention, age, and conscious state. PloS One 13, e0206237. doi:10.1371/journal.pone.0206237.

Ismael, J. (2016). How Physics Makes Us Free. Oxford University Press.

Izquierdo, E., Aguilera, M., and Beer, R. (2013). Analysis of Ultrastability in Small Dynamical Recurrent Neural Networks. 2018 Conf. Artif. Life Hybrid Eur. Conf. Artif. Life ECAL Int. Conf. Synth. Simul. Living Syst. ALIFE 25, 51-58. doi:10.1162/978-0-262-31709-2-ch008.

Izquierdo-Torres, E., and Bührmann, T. (2008). Analysis of a Dynamical Recurrent Neural Network Evolved for Two Qualitatively Different Tasks: Walking and Chemotaxis. in ALIFE.

James, W. (1879). Are We Automata? Mind 4, 1-22. doi:10.1093/mind/os-4.13.1.

James, W. (1890). The Principles of Psychology, Vol. 1. Reprint edition. New York: Dover Publications.

Jaynes, J. (1976). The Origin of Consciousness in the Breakdown of the Bicameral Mind. Houghton Mifflin Harcourt.

Jennings, J. R., Allen, B., Gianaros, P. J., Thayer, J. F., and Manuck, S. B. (2015). Focusing neurovisceral integration: Cognition, heart rate variability, and cerebral blood flow. Psychophysiology 52, 214-224. doi:10.1111/psyp.12319.

Joffily, M., and Coricelli, G. (2013). Emotional valence and the free-energy principle. PLoS Comput. Biol. 9, e1003094. doi:10.1371/journal.pcbi.1003094.

Joslyn, null (2000). Levels of control and closure in complex semiotic systems. Ann. N. Y. Acad. Sci. 901, 67-74.

Kachman, T., Owen, J. A., and England, J. L. (2017). Self-Organized Resonance during Search of a Diverse Chemical Space. Phys. Rev. Lett. 119, 038001. doi:10.1103/PhysRevLett.119.038001.

Kahneman, D. (2011). Thinking, Fast and Slow. 1st ed. Farrar, Straus and Giroux.

Kaila, V., and Annila, A. (2008). Natural selection for least action. Proc. R. Soc. Math. Phys. Eng. Sci. 464, 30553070. doi:10.1098/rspa.2008.0178.

Kalra, P. B., Gabrieli, J. D. E., and Finn, A. S. (2019). Evidence of stable individual differences in implicit learning. Cognition 190, 199-211. doi:10.1016/j.cognition.2019.05.007.

Kanai, R., Chang, A., Yu, Y., Magrans de Abril, I., Biehl, M., and Guttenberg, N. (2019). Information generation as a functional basis of consciousness. Neurosci. Conscious. 2019. doi:10.1093/nc/niz016.

Kant, I. (1781). Critique of Pure Reason., eds. P. Guyer and A. W. Wood Cambridge: Cambridge University Press.

Kaplan, R., and Friston, K. J. (2018). Planning and navigation as active inference. Biol. Cybern. 112, 323-343. doi:10.1007/s00422-018-0753-2.

Kauffman, S. A. (2014). Prolegomenon to patterns in evolution. Biosystems 123, 3-8. doi:10.1016/j.biosystems.2014.03.004.

Kauffman, S., and Clayton, P. (2006). On emergence, agency, and organization. Biol. Philos. 21, 501-521. doi:10.1007/s10539-005-9003-9.

Keogh, R., and Pearson, J. (2018). The blind mind: No sensory visual imagery in aphantasia. Cortex 105, 53-60. doi:10.1016/j.cortex.2017.10.012.

Kilteni, K., Andersson, B. J., Houborg, C., and Ehrsson, H. H. (2018). Motor imagery involves predicting the sensory consequences of the imagined movement. Nat. Commun. 9, 1-9. doi:10.1038/s41467-018-03989-0.

Kim, S. S., Hermundstad, A. M., Romani, S., Abbott, L. F., and Jayaraman, V. (2019). Generation of stable heading representations in diverse visual scenes. Nature 576, 126-131. doi:10.1038/s41586-019-1767-1.

Kingma, D. P., and Welling, M. (2014). Auto-Encoding Variational Bayes. ArXiv13126114 Cs Stat. Available at: http://arxiv.org/abs/1312.6114 [Accessed March 29, 2020].

Kinouchi, O., Brochini, L., Costa, A. A., Campos, J. G. F., and Copelli, M. (2019). Stochastic oscillations and dragon king avalanches in self-organized quasi-critical systems. Sci. Rep. 9, 1-12. doi:10.1038/s41598019-40473-1.

Kirchhoff, M., Parr, T., Palacios, E., Friston, K. J., and Kiverstein, J. (2018). The Markov blankets of life: autonomy, active inference and the free energy principle. J. R. Soc. Interface 15. doi:10.1098/rsif.2017.0792.

Kiverstein, J., Miller, M., and Rietveld, E. (2019). The feeling of grip: novelty, error dynamics, and the predictive brain. Synthese 196, 2847-2869. doi:10.1007/s11229-017-1583-9.

Knight, R. T., and Grabowecky, M. (1995). "Escape from linear time: Prefrontal cortex and conscious experience," in The cognitive neurosciences (Cambridge, MA, US: The MIT Press), 1357-1371.

Koch, C. (2012). Consciousness: Confessions of a Romantic Reductionist. MIT Press. 
Koelsch, S., Vuust, P., and Friston, K. J. (2019). Predictive Processes and the Peculiar Case of Music. Trends Cogn. Sci. 23, 63-77. doi:10.1016/j.tics.2018.10.006.

Koller, D., and Friedman, N. (2009). Probabilistic Graphical Models: Principles and Techniques. MIT Press.

Kosiorek, A., Sabour, S., Teh, Y. W., and Hinton, G. E. (2019). "Stacked Capsule Autoencoders," in Advances in Neural Information Processing Systems 32, eds. H. Wallach, H. Larochelle, A. Beygelzimer, F. dltextquotesingle Alché-Buc, E. Fox, and R. Garnett (Curran Associates, Inc.), 15512-15522. Available at: http://papers.nips.cc/paper/9684-stacked-capsule-autoencoders.pdf [Accessed May 14, 2020].

Koster, R., Chadwick, M. J., Chen, Y., Berron, D., Banino, A., Düzel, E., et al. (2018). Big-Loop Recurrence within the Hippocampal System Supports Integration of Information across Episodes. Neuron 99, 1342-1354.e6. doi:10.1016/j.neuron.2018.08.009.

Kramer, M. A. (1991). Nonlinear principal component analysis using autoassociative neural networks. AIChE J. 37, 233-243. doi:10.1002/aic.690370209.

Kropff, E., and Treves, A. (2008). The emergence of grid cells: Intelligent design or just adaptation? Hippocampus 18, 1256-1269. doi:10.1002/hipo.20520.

Kryven, I. (2019). Bond percolation in coloured and multiplex networks. Nat. Commun. 10, 1-16. doi:10.1038/s41467-018-08009-9.

Kunz, L., Wang, L., Lachner-Piza, D., Zhang, H., Brandt, A., Dümpelmann, M., et al. (2019). Hippocampal theta phases organize the reactivation of large-scale electrophysiological representations during goal-directed navigation. Sci. Adv. 5, eaav8192. doi:10.1126/sciadv.aav8192.

Kushnir, T. (2018). The developmental and cultural psychology of free will. Philos. Compass 13, e12529. doi:10.1111/phc3.12529.

Kushnir, T., Gopnik, A., Chernyak, N., Seiver, E., and Wellman, H. M. (2015). Developing intuitions about free will between ages four and six. Cognition 138, 79-101. doi:10.1016/j.cognition.2015.01.003.

Lahav, N., Sendiña-Nadal, I., Hens, C., Ksherim, B., Barzel, B., Cohen, R., et al. (2018). Synchronization of chaotic systems: A microscopic description. Phys. Rev. E 98, 052204. doi:10.1103/PhysRevE.98.052204.

Lakoff, G. (2014). Mapping the brain's metaphor circuitry: metaphorical thought in everyday reason. Front. Hum. Neurosci. 8. doi:10.3389/fnhum.2014.00958.

Lakoff, G., and Johnson, M. (1999). Philosophy in the Flesh : The Embodied Mind and Its Challenge to Western Thought. Basic Books.

Lau, H., and Michel, M. (2019). On the dangers of conflating strong and weak versions of a theory of consciousness. PsyArXiv doi:10.31234/osf.io/hjp3s.

LeDoux, J. (2016). Anxious: Using the Brain to Understand and Treat Fear and Anxiety. Reprint edition. Penguin Books.

LeDoux, J. (2019). The Deep History of Ourselves: The Four-Billion-Year Story of How We Got Conscious Brains. Viking.

Lee, A. M., Hoy, J. L., Bonci, A., Wilbrecht, L., Stryker, M. P., and Niell, C. M. (2014). Identification of a brainstem circuit regulating visual cortical state in parallel with locomotion. Neuron 83, 455-466. doi:10.1016/j.neuron.2014.06.031.

Legg, S., and Hutter, M. (2006). A formal measure of machine intelligence. ArXiv Prepr. Cs0605024.

Legg, S., and Hutter, M. (2007). A collection of definitions of intelligence. Front. Artif. Intell. Appl. 157, 17.

Leknes, S., and Tracey, I. (2008). A common neurobiology for pain and pleasure. Nat. Rev. Neurosci. 9, 314-320. doi:10.1038/nrn2333.

Levin, I. (1992). "The Development of the Concept of Time in Children: An Integrative Model," in Time, Action and Cognition: Towards Bridging the Gap NATO ASI Series., eds. F. Macar, V. Pouthas, and W. J. Friedman (Dordrecht: Springer Netherlands), 13-32. doi:10.1007/978-94-017-3536-0_3.

Levin, I., Israeli, E., and Darom, E. (1978). The Development of Time Concepts in Young Children: The Relations between Duration and Succession. Child Dev. 49, 755-764. doi:10.2307/1128245.

Lewis, C. S. (1960). Studies in Words. Cambridge University Press.

Lewis, M. (2015). The biology of desire: Why addiction is not a disease. New York, NY, US: Public Affairs Books.

Li, M., Woelfer, M., Colic, L., Safron, A., Chang, C., Heinze, H.-J., et al. (2018). Default mode network connectivity change corresponds to ketamine's delayed glutamatergic effects. Eur. Arch. Psychiatry Clin. Neurosci. doi:10.1007/s00406-018-0942-y.

Libet, B., Gleason, C. A., Wright, E. W., and Pearl, D. K. (1983). Time of conscious intention to act in relation to onset of cerebral activity (readiness-potential). The unconscious initiation of a freely voluntary act. Brain J. Neurol. 106 (Pt 3), 623-642. doi:10.1093/brain/106.3.623. 
Lillicrap, T. P., Santoro, A., Marris, L., Akerman, C. J., and Hinton, G. (2020). Backpropagation and the brain. Nat. Rev. Neurosci., 1-12. doi:10.1038/s41583-020-0277-3.

Limanowski, J., and Friston, K. J. (2018). 'Seeing the Dark': Grounding Phenomenal Transparency and Opacity in Precision Estimation for Active Inference. Front. Psychol. 9. doi:10.3389/fpsyg.2018.00643.

Lind, J. (2018). What can associative learning do for planning? in Royal Society open science doi:10.1098/rsos.180778.

Linson, A., Clark, A., Ramamoorthy, S., and Friston, K. J. (2018). The active inference approach to ecological perception: general information dynamics for natural and artificial embodied cognition. Front. Robot. AI 5, 21.

Liu, J., Kumar, A., Ba, J., Kiros, J., and Swersky, K. (2019). Graph Normalizing Flows. ArXiv190513177 Cs Stat. Available at: http://arxiv.org/abs/1905.13177 [Accessed May 24, 2020].

Liu, Y.-H., and Poulin, D. (2019). Neural Belief-Propagation Decoders for Quantum Error-Correcting Codes. Phys. Rev. Lett. 122, 200501. doi:10.1103/PhysRevLett.122.200501.

Lloyd, S. (2012). A Turing test for free will. Philos. Trans. R. Soc. Math. Phys. Eng. Sci. 370, 3597-3610. doi:10.1098/rsta.2011.0331.

Lord, L.-D., Expert, P., Atasoy, S., Roseman, L., Rapuano, K., Lambiotte, R., et al. (2019). Dynamical exploration of the repertoire of brain networks at rest is modulated by psilocybin. NeuroImage 199, 127-142. doi:10.1016/j.neuroimage.2019.05.060.

MacIver, M. A., Schmitz, L., Mugan, U., Murphey, T. D., and Mobley, C. D. (2017). Massive increase in visual range preceded the origin of terrestrial vertebrates. Proc. Natl. Acad. Sci. 114, E2375-E2384. doi:10.1073/pnas.1615563114.

MacKay, D. G. (2019). Remembering: What 50 Years of Research with Famous Amnesia Patient H. M. Can Teach Us about Memory and How It Works. Prometheus Books.

Madl, T., Baars, B. J., and Franklin, S. (2011). The timing of the cognitive cycle. PloS One 6, e14803.

Mahr, J., and Csibra, G. (2017). Why do we remember? The communicative function of episodic memory. Behav. Brain Sci., 1-93. doi:10.1017/S0140525X17000012.

Mannella, F., Gurney, K., and Baldassarre, G. (2013). The nucleus accumbens as a nexus between values and goals in goal-directed behavior: a review and a new hypothesis. Front. Behav. Neurosci. 7, 135. doi:10.3389/fnbeh.2013.00135.

Manning, J. R., Sperling, M. R., Sharan, A., Rosenberg, E. A., and Kahana, M. J. (2012). Spontaneously Reactivated Patterns in Frontal and Temporal Lobe Predict Semantic Clustering during Memory Search. J. Neurosci. 32, 8871-8878. doi:10.1523/JNEUROSCI.5321-11.2012.

Maoz, U., Yaffe, G., Koch, C., and Mudrik, L. (2019). Neural precursors of decisions that matter-an ERP study of deliberate and arbitrary choice. eLife 8. doi:10.7554/eLife.39787.

Marcus, G., and Davis, E. (2019). Rebooting AI: Building Artificial Intelligence We Can Trust. Knopf Doubleday Publishing Group.

Markram, H., Gerstner, W., and Sjöström, P. J. (2011). A history of spike-timing-dependent plasticity. Front. Synaptic Neurosci. 3, 4. doi:10.3389/fnsyn.2011.00004.

Marshall, P. J., and Meltzoff, A. N. (2015). Body maps in the infant brain. Trends Cogn. Sci. 19, 499-505. doi:10.1016/j.tics.2015.06.012.

Marshall, W., Gomez-Ramirez, J., and Tononi, G. (2016). Integrated Information and State Differentiation. Front. Psychol. 7, 926. doi:10.3389/fpsyg.2016.00926.

Martik, M. L., Gandhi, S., Uy, B. R., Gillis, J. A., Green, S. A., Simoes-Costa, M., et al. (2019). Evolution of the new head by gradual acquisition of neural crest regulatory circuits. Nature 574, 675-678. doi:10.1038/s41586-019-1691-4.

Maschler, M. (1992). The bargaining set, kernel, and nucleolus. Handb. Game Theory Econ. Appl. 1, 591-667.

Maschler, M., Peleg, B., and Shapley, L. S. (1979). Geometric properties of the kernel, nucleolus, and related solution concepts. Math. Oper. Res. 4, 303-338.

Mashour, G. A., Roelfsema, P., Changeux, J.-P., and Dehaene, S. (2020). Conscious Processing and the Global Neuronal Workspace Hypothesis. Neuron 105, 776-798. doi:10.1016/j.neuron.2020.01.026.

Massimini, M., Ferrarelli, F., Sarasso, S., and Tononi, G. (2012). Cortical mechanisms of loss of consciousness: insight from TMS/EEG studies. Arch. Ital. Biol. 150, 44-55.

Mayner, W. G. P., Marshall, W., Albantakis, L., Findlay, G., Marchman, R., and Tononi, G. (2018). PyPhi: A toolbox for integrated information theory. PLOS Comput. Biol. 14, e1006343. doi:10.1371/journal.pcbi.1006343. 
McCulloch, W. S., and Pitts, W. (1943). A logical calculus of the ideas immanent in nervous activity. Bull. Math. Biophys. 5, 115-133.

McEliece, R. J., MacKay, D. J. C., and Jung-Fu Cheng (1998). Turbo decoding as an instance of Pearl's "belief propagation" algorithm. IEEE J. Sel. Areas Commun. 16, 140-152. doi:10.1109/49.661103.

McGilchrist, I. (2019). The Master and His Emissary: The Divided Brain and the Making of the Western World. Yale University Press.

McGurk, H., and MacDonald, J. (1976). Hearing lips and seeing voices. Nature 264, 746-748.

McNamara, C. G., and Dupret, D. (2017). Two sources of dopamine for the hippocampus. Trends Neurosci. 40, 383-384. doi:10.1016/j.tins.2017.05.005.

Mediano, P. A. M., Rosas, F., Carhart-Harris, R. L., Seth, A. K., and Barrett, A. B. (2019a). Beyond integrated information: A taxonomy of information dynamics phenomena. ArXiv190902297 Phys. Q-Bio. Available at: http://arxiv.org/abs/1909.02297 [Accessed November 23, 2019].

Mediano, P. A. M., Seth, A. K., and Barrett, A. B. (2019b). Measuring Integrated Information: Comparison of Candidate Measures in Theory and Simulation. Entropy 21, 17. doi:10.3390/e21010017.

Metzinger, T. (2010). The Ego Tunnel: The Science of the Mind and the Myth of the Self. ReadHowYouWant.com.

Miller, L. E., Fabio, C., Ravenda, V., Bahmad, S., Koun, E., Salemme, R., et al. (2019). Somatosensory Cortex Efficiently Processes Touch Located Beyond the Body. Curr. Biol. doi:10.1016/j.cub.2019.10.043.

Minsky, M. (1988). Society Of Mind. Simon and Schuster.

Mišić, B., Betzel, R. F., Nematzadeh, A., Goñi, J., Griffa, A., Hagmann, P., et al. (2015). Cooperative and Competitive Spreading Dynamics on the Human Connectome. Neuron 86, 1518-1529. doi:10.1016/j.neuron.2015.05.035.

Mitchell, M. (2019). Artificial Intelligence: A Guide for Thinking Humans. Farrar, Straus and Giroux.

Morgan, A. T., Petro, L. S., and Muckli, L. (2019). Line drawings reveal the structure of internal visual models conveyed by cortical feedback. bioRxiv, 041186. doi:10.1101/041186.

Moser, E. I., Kropff, E., and Moser, M.-B. (2008). Place cells, grid cells, and the brain's spatial representation system. Annu. Rev. Neurosci. 31, 69-89. doi:10.1146/annurev.neuro.31.061307.090723.

Mugan, U., and MacIver, M. A. (2019). The shift from life in water to life on land advantaged planning in visuallyguided behavior. bioRxiv, 585760. doi:10.1101/585760.

Muller, L., Chavane, F., Reynolds, J., and Sejnowski, T. J. (2018). Cortical travelling waves: mechanisms and computational principles. Nat. Rev. Neurosci. 19, 255-268. doi:10.1038/nrn.2018.20.

Musall, S., Kaufman, M. T., Juavinett, A. L., Gluf, S., and Churchland, A. K. (2019). Single-trial neural dynamics are dominated by richly varied movements. Nat. Neurosci. 22, 1677-1686. doi:10.1038/s41593-019-05024.

Nagel, T. (1974). What Is It Like to Be a Bat? Philos. Rev. 83, 435-450. doi:10.2307/2183914.

Nau, M., Schröder, T. N., Bellmund, J. L. S., and Doeller, C. F. (2018). Hexadirectional coding of visual space in human entorhinal cortex. Nat. Neurosci. 21, 188-190. doi:10.1038/s41593-017-0050-8.

Noë, A. (2002). Is the visual world a grand illusion? J. Conscious. Stud. 9, 1-12.

Ocaña, F. M., Suryanarayana, S. M., Saitoh, K., Kardamakis, A. A., Capantini, L., Robertson, B., et al. (2015). The Lamprey Pallium Provides a Blueprint of the Mammalian Motor Projections from Cortex. Curr. Biol. 25, 413-423. doi:10.1016/j.cub.2014.12.013.

Oliveira, H. M., and Melo, L. V. (2015). Huygens synchronization of two clocks. Sci. Rep. 5, 1-12. doi:10.1038/srep11548.

O'Regan, J. K., and Noë, A. (2001). A sensorimotor account of vision and visual consciousness. Behav. Brain Sci. 24, 939-973; discussion 973-1031. doi:10.1017/s0140525x01000115.

O'Reilly, R. C., Wyatte, D. R., and Rohrlich, J. (2017). Deep Predictive Learning: A Comprehensive Model of Three Visual Streams. ArXiv170904654 Q-Bio. Available at: http://arxiv.org/abs/1709.04654 [Accessed October 19, 2019].

Palacios, E. R., Isomura, T., Parr, T., and Friston, K. J. (2019). The emergence of synchrony in networks of mutually inferring neurons. Sci. Rep. 9, 6412. doi:10.1038/s41598-019-42821-7.

Panksepp, J. (1998). Affective Neuroscience: The Foundations of Human and Animal Emotions. illustrated edition. Oxford University Press, USA.

Panksepp, J. (2005). Affective consciousness: Core emotional feelings in animals and humans. Conscious. Cogn. 14, 30-80. doi:10.1016/j.concog.2004.10.004.

Panksepp, J. (2007). Neuroevolutionary sources of laughter and social joy: modeling primal human laughter in laboratory rats. Behav. Brain Res. 182, 231-244. doi:10.1016/j.bbr.2007.02.015. 
Paperin, G., Green, D. G., and Sadedin, S. (2011). Dual-phase evolution in complex adaptive systems. J. R. Soc. Interface 8, 609-629. doi:10.1098/rsif.2010.0719.

Papez, J.W. (1937). A proposed mechanism of emotion. Arch. Neurol. Psychiatry 38, 725-743. doi:10.1001/archneurpsyc.1937.02260220069003.

Parr, T., Corcoran, A. W., Friston, K. J., and Hohwy, J. (2019a). Perceptual awareness and active inference. Neurosci. Conscious. 2019. doi:10.1093/nc/niz012.

Parr, T., and Friston, K. J. (2018a). The Anatomy of Inference: Generative Models and Brain Structure. Front. Comput. Neurosci. 12, 90. doi:10.3389/fncom.2018.00090.

Parr, T., and Friston, K. J. (2018b). The Discrete and Continuous Brain: From Decisions to Movement-And Back Again. Neural Comput. 30, 2319-2347. doi:10.1162/neco_a_01102.

Parr, T., Markovic, D., Kiebel, S. J., and Friston, K. J. (2019b). Neuronal message passing using Mean-field, Bethe, and Marginal approximations. Sci. Rep. 9. doi:10.1038/s41598-018-38246-3.

Parr, T., Rikhye, R. V., Halassa, M. M., and Friston, K. J. (2019c). Prefrontal computation as active inference. Cereb. Cortex.

Pearl, J. (1982). Reverend Bayes on inference engines: A distributed hierarchical approach. Cognitive Systems Laboratory, School of Engineering and Applied Science ....

Pearl, J., and Mackenzie, D. (2018). The Book of Why: The New Science of Cause and Effect. Basic Books.

Peña-Gómez, C., Avena-Koenigsberger, A., Sepulcre, J., and Sporns, O. (2018). Spatiotemporal Network Markers of Individual Variability in the Human Functional Connectome. Cereb. Cortex 28, 2922-2934. doi:10.1093/cercor/bhx170.

Peterson, J. B. (1999). Maps of Meaning: The Architecture of Belief. Psychology Press.

Pezzulo, G., Rigoli, F., and Friston, K. J. (2018). Hierarchical Active Inference: A Theory of Motivated Control. Trends Cogn. Sci. 22, 294-306. doi:10.1016/j.tics.2018.01.009.

Pfeifer, R., and Bongard, J. (2006). How the Body Shapes the Way We Think: A New View of Intelligence. Cambridge, Mass: A Bradford Book.

Pfeiffer, K., and Homberg, U. (2014). Organization and Functional Roles of the Central Complex in the Insect Brain. Annu. Rev. Entomol. 59, 165-184. doi:10.1146/annurev-ento-011613-162031.

Porges, S. W. (2009). The polyvagal theory: New insights into adaptive reactions of the autonomic nervous system. Cleve. Clin. J. Med. 76, S86-S90. doi:10.3949/ccjm.76.s2.17.

Prinz, J. (2017). "The Intermediate Level Theory of Consciousness," in The Blackwell Companion to Consciousness (John Wiley \& Sons, Ltd), 257-271. doi:10.1002/9781119132363.ch18.

Ramachandran, V. S., Blakeslee, S., and Sacks, O. (1999). Phantoms in the Brain: Probing the Mysteries of the Human Mind. New York: William Morrow Paperbacks.

Ramstead, M. J. D., Badcock, P. B., and Friston, K. J. (2017). Answering Schrödinger's question: A free-energy formulation. Phys. Life Rev. doi:10.1016/j.plrev.2017.09.001.

Ramstead, M. J. D., Kirchhoff, M. D., and Friston, K. J. (2019). A tale of two densities: Active inference is enactive inference. Available at: http://philsci-archive.pitt.edu/16167/ [Accessed December 12, 2019].

Redgrave, P., Gurney, K., and Reynolds, J. (2008). What is reinforced by phasic dopamine signals? Brain Res. Rev. 58, 322-339. doi:10.1016/j.brainresrev.2007.10.007.

Richards, B. A., Lillicrap, T. P., Beaudoin, P., Bengio, Y., Bogacz, R., Christensen, A., et al. (2019). A deep learning framework for neuroscience. Nat. Neurosci. 22, 1761-1770. doi:10.1038/s41593-019-0520-2.

Roberts, J. A., Gollo, L. L., Abeysuriya, R. G., Roberts, G., Mitchell, P. B., Woolrich, M. W., et al. (2019). Metastable brain waves. Nat. Commun. 10, 1-17. doi:10.1038/s41467-019-08999-0.

Rocha, L. M. (2000). Syntactic autonomy. Why there is no autonomy without symbols and how self-organizing systems might evolve them. Ann. N. Y. Acad. Sci. 901, 207-223. doi:10.1111/j.1749-6632.2000.tb06280.x.

Rochat, P. (2010). "Emerging Self-Concept," in The Wiley-Blackwell Handbook of Infant Development, eds. J. G. Bremner and T. D. Wachs (Wiley-Blackwell), 320-344. doi:10.1002/9781444327564.ch10.

Roepstorff, A., and Frith, C. (2004). What's at the top in the top-down control of action? Script-sharing and "toptop" control of action in cognitive experiments. Psychol. Res. 68, 189-198. doi:10.1007/s00426-003-01554.

Rudrauf, D., Bennequin, D., Granic, I., Landini, G., Friston, K. J., and Williford, K. (2017). A mathematical model of embodied consciousness. J. Theor. Biol. 428, 106-131. doi:10.1016/j.jtbi.2017.05.032.

Russon, A. E., and Begun, D. R. (2007). The Evolution of Thought: Evolutionary Origins of Great Ape Intelligence. Cambridge University Press.

Sacks, O. (2008). Musicophilia: Tales of Music and the Brain, Revised and Expanded Edition. Revised\&enlarged edition. New York: Vintage. 
Sacks, O. (2013). Hallucinations. 1st edition. Waterville, Maine: Vintage.

Safron, A. (2016). What is orgasm? A model of sexual trance and climax via rhythmic entrainment. Socioaffective Neurosci. Psychol. 6, 31763.

Safron, A. (2019a). Bayesian Analogical Cybernetics. ArXiv191102362 Q-Bio. Available at: http://arxiv.org/abs/1911.02362 [Accessed November 14, 2019].

Safron, A. (2019b). Multilevel evolutionary developmental optimization (MEDO): A theoretical framework for understanding preferences and selection dynamics. ArXiv191013443 Econ Q-Bio Q-Fin. Available at: http://arxiv.org/abs/1910.13443 [Accessed November 14, 2019].

Safron, A. (2019c). Rapid Anxiety Reduction (RAR): A unified theory of humor. ArXiv191102364 Q-Bio. Available at: http://arxiv.org/abs/1911.02364 [Accessed November 14, 2019].

Safron, A. (2019d). The radically embodied conscious cybernetic Bayesian brain: Towards explaining the emergence of agency. doi:10.31234/osf.io/udc42.

Safron, A. (2020). An Integrated World Modeling Theory (IWMT) of Consciousness: Combining Integrated Information and Global Neuronal Workspace Theories With the Free Energy Principle and Active Inference Framework; Toward Solving the Hard Problem and Characterizing Agentic Causation. Front. Artif. Intell. 3. doi:10.3389/frai.2020.00030.

Sagan, C. (1977). The Dragons of Eden: Speculations on the Evolution of Human Intelligence. Ballantine Books. Sandrone, S., and Safron, A. (2013). Pain and (e) motion in postural responses. Front. Hum. Neurosci. 7, 286.

Sarel, A., Finkelstein, A., Las, L., and Ulanovsky, N. (2017). Vectorial representation of spatial goals in the hippocampus of bats. Science 355, 176-180. doi:10.1126/science.aak9589.

Sasai, S., Boly, M., Mensen, A., and Tononi, G. (2016). Functional split brain in a driving/listening paradigm. Proc. Natl. Acad. Sci. 113, 14444-14449. doi:10.1073/pnas.1613200113.

Sayette, M. A., Loewenstein, G., Griffin, K. M., and Black, J. J. (2008). Exploring the cold-to-hot empathy gap in smokers. Psychol. Sci. 19, 926-932. doi:10.1111/j.1467-9280.2008.02178.x.

Schartner, M. M., Carhart-Harris, R. L., Barrett, A. B., Seth, A. K., and Muthukumaraswamy, S. D. (2017). Increased spontaneous MEG signal diversity for psychoactive doses of ketamine, LSD and psilocybin. Sci. Rep. 7, 46421. doi:10.1038/srep46421.

Scheeringa, R., and Fries, P. (2019). Cortical layers, rhythms and BOLD signals. NeuroImage 197, 689-698. doi:10.1016/j.neuroimage.2017.11.002.

Schmeidler, D. (1969). The nucleolus of a characteristic function game. SIAM J. Appl. Math. 17, 1163-1170.

Schmidhuber, J. (2012). POWERPLAY: Training an Increasingly General Problem Solver by Continually Searching for the Simplest Still Unsolvable Problem. ArXiv11125309 Cs. Available at: http://arxiv.org/abs/1112.5309 [Accessed November 24, 2019].

Schopenhauer, A. (1844). The World as Will and Representation. Courier Corporation.

Schultz, W. (2010). Dopamine signals for reward value and risk: basic and recent data. Behav. Brain Funct. BBF 6, 24. doi:10.1186/1744-9081-6-24.

Schultz, W. (2016). Dopamine reward prediction error coding. Dialogues Clin. Neurosci. 18, 23-32.

Schurger, A., Sitt, J. D., and Dehaene, S. (2012). An accumulator model for spontaneous neural activity prior to selfinitiated movement. Proc. Natl. Acad. Sci. 109, E2904-E2913. doi:10.1073/pnas.1210467109.

Seth, A. K. (2014a). A predictive processing theory of sensorimotor contingencies: Explaining the puzzle of perceptual presence and its absence in synesthesia. Cogn. Neurosci. 5, 97-118. doi:10.1080/17588928.2013.877880.

Seth, A. K. (2014b). A predictive processing theory of sensorimotor contingencies: Explaining the puzzle of perceptual presence and its absence in synesthesia. Cogn. Neurosci. 5, 97-118. doi:10.1080/17588928.2013.877880.

Seth, A. K. (2014c). The Cybernetic Bayesian Brain. Open MIND. Frankfurt am Main: MIND Group doi:10.15502/9783958570108.

Seth, A. K. (2016). The hard problem of consciousness is a distraction from the real one - Anil K Seth | Aeon Essays. Aeon. Available at: https://aeon.co/essays/the-hard-problem-of-consciousness-is-a-distractionfrom-the-real-one [Accessed November 25, 2019].

Seth, A. K., Barrett, A. B., and Barnett, L. (2011a). Causal density and integrated information as measures of conscious level. Philos. Transact. A Math. Phys. Eng. Sci. 369, 3748-3767. doi:10.1098/rsta.2011.0079.

Seth, A. K., and Friston, K. J. (2016). Active interoceptive inference and the emotional brain. Phil Trans $R$ Soc B 371, 20160007. doi:10.1098/rstb.2016.0007.

Seth, A. K., Suzuki, K., and Critchley, H. D. (2011b). An interoceptive predictive coding model of conscious presence. Front. Psychol. 2, 395. doi:10.3389/fpsyg.2011.00395. 
Seth, A. K., Suzuki, K., and Critchley, H. D. (2012). An Interoceptive Predictive Coding Model of Conscious Presence. Front. Psychol. 2. doi:10.3389/fpsyg.2011.00395.

Seth, A. K., and Tsakiris, M. (2018). Being a Beast Machine: The Somatic Basis of Selfhood. Trends Cogn. Sci. 22, 969-981. doi:10.1016/j.tics.2018.08.008.

Shanahan, M. (2012). The brain's connective core and its role in animal cognition. Philos. Trans. R. Soc. B Biol. Sci. 367, 2704-2714. doi:10.1098/rstb.2012.0128.

Shanahan, M., and Baars, B. (2005). Applying global workspace theory to the frame problem. Cognition 98, 157176. doi:10.1016/j.cognition.2004.11.007.

Shea, N., and Frith, C. D. (2019). The Global Workspace Needs Metacognition. Trends Cogn. Sci. 0. doi:10.1016/j.tics.2019.04.007.

Shiller, R. J. (2019). Narrative Economics: How Stories Go Viral and Drive Major Economic Events. Princeton: Princeton University Press.

Sievers, B., Lee, C., Haslett, W., and Wheatley, T. (2019). A multi-sensory code for emotional arousal. Proc. R. Soc. B Biol. Sci. 286, 20190513. doi:10.1098/rspb.2019.0513.

Sievers, B., Polansky, L., Casey, M., and Wheatley, T. (2013). Music and movement share a dynamic structure that supports universal expressions of emotion. Proc. Natl. Acad. Sci. U. S. A. 110, 70-75. doi:10.1073/pnas.1209023110.

Singer, W. (2001). Consciousness and the binding problem. Ann. N. Y. Acad. Sci. 929, 123-146.

Singer, W. (2007). Phenomenal Awareness and Consciousness from a Neurobiological Perspective. NeuroQuantology 4. doi:10.14704/nq.2006.4.2.94.

Sleigh, R. (2003). "GW Leibniz, Monadology (1714)," in The Classics of Western Philosophy: A Reader's Guide, eds. J. J. E. Gracia, G. M. Reichberg, and B. N. Schumacher (Blackwell), 277.

Smith, G. B., Hein, B., Whitney, D. E., Fitzpatrick, D., and Kaschube, M. (2018a). Distributed network interactions and their emergence in developing neocortex. Nat. Neurosci. 21, 1600-1608. doi:10.1038/s41593-0180247-5.

Smith, L. B., Jayaraman, S., Clerkin, E., and Yu, C. (2018b). The Developing Infant Creates a Curriculum for Statistical Learning. Trends Cogn. Sci. 22, 325-336. doi:10.1016/j.tics.2018.02.004.

Soares, S., Atallah, B. V., and Paton, J. J. (2016). Midbrain dopamine neurons control judgment of time. Science 354, 1273-1277. doi:10.1126/science.aah5234.

Solms, M., and Friston, K. J. (2018). How and Why Consciousness Arises: Some Considerations from Physics and Physiology. Available at: https://www.ingentaconnect.com/content/imp/jcs/2018/00000025/f0020005/art00009 [Accessed June 15, 2019].

Sormaz, M., Murphy, C., Wang, H., Hymers, M., Karapanagiotidis, T., Poerio, G., et al. (2018). Default mode network can support the level of detail in experience during active task states. Proc. Natl. Acad. Sci. 115, 9318-9323. doi:10.1073/pnas.1721259115.

Spelke, E. S., and Kinzler, K. D. (2007). Core knowledge. Dev. Sci. 10, 89-96. doi:10.1111/j.14677687.2007.00569.x.

Srivastava, N., Hinton, G., Krizhevsky, A., Sutskever, I., and Salakhutdinov, R. (2014). Dropout: a simple way to prevent neural networks from overfitting. J. Mach. Learn. Res. 15, 1929-1958.

St. Jacques, P. L. (2019). A New Perspective on Visual Perspective in Memory. Curr. Dir. Psychol. Sci. 28, 450455. doi:10.1177/0963721419850158.

Steppa, C., and Holch, T. L. (2019). HexagDLy-Processing hexagonally sampled data with CNNs in PyTorch. SoftwareX 9, 193-198. doi:10.1016/j.softx.2019.02.010.

Stolyarova, A. (2018). Solving the Credit Assignment Problem With the Prefrontal Cortex. Front. Neurosci. 12. doi:10.3389/fnins.2018.00182.

Strogatz, S. H. (2012). Sync: How Order Emerges from Chaos In the Universe, Nature, and Daily Life. Hachette Books.

Sundberg, M. L. (2013). Thirty Points About Motivation From Skinner's Book Verbal Behavior. Anal. Verbal Behav. 29, 13-40.

Suryanarayana, S. M., Pérez-Fernández, J., Robertson, B., and Grillner, S. (2020). The evolutionary origin of visual and somatosensory representation in the vertebrate pallium. Nat. Ecol. Evol., 1-13. doi:10.1038/s41559020-1137-2.

Suryanarayana, S. M., Robertson, B., Wallén, P., and Grillner, S. (2017). The Lamprey Pallium Provides a Blueprint of the Mammalian Layered Cortex. Curr. Biol. CB 27, 3264-3277.e5. doi:10.1016/j.cub.2017.09.034. 
Suzuki, K., Lush, P., Seth, A. K., and Roseboom, W. (2019). Intentional Binding Without Intentional Action. Psychol. Sci. 30, 842-853. doi:10.1177/0956797619842191.

Swanson, L. R. (2016). The Predictive Processing Paradigm Has Roots in Kant. Front. Syst. Neurosci. 10, 79. doi:10.3389/fnsys.2016.00079.

Takagi, K. (2018). Information-Based Principle Induces Small-World Topology and Self-Organized Criticality in a Large Scale Brain Network. Front. Comput. Neurosci. 12. doi:10.3389/fncom.2018.00065.

Tani, J. (2016). Exploring robotic minds: actions, symbols, and consciousness as self-organizing dynamic phenomena. Oxford University Press.

Taylor, A. H., Knaebe, B., and Gray, R. D. (2012). An end to insight? New Caledonian crows can spontaneously solve problems without planning their actions. Proc. Biol. Sci. 279, 4977-4981. doi:10.1098/rspb.2012.1998.

Tegmark, M. (2000). The importance of quantum decoherence in brain processes. Phys. Rev. E 61, 4194-4206. doi:10.1103/PhysRevE.61.4194.

Tegmark, M. (2016). Improved Measures of Integrated Information. PLoS Comput. Biol. 12. doi:10.1371/journal.pcbi.1005123.

Tenenbaum, J. B., Kemp, C., Griffiths, T. L., and Goodman, N. D. (2011). How to Grow a Mind: Statistics, Structure, and Abstraction. Science 331, 1279-1285. doi:10.1126/science.1192788.

Terekhov, A. V., and O’Regan, J. K. (2013). Space as an invention of biological organisms. ArXiv13082124 Cs. Available at: http://arxiv.org/abs/1308.2124 [Accessed November 24, 2019].

Terekhov, A. V., and O'Regan, J. K. (2016). Space as an Invention of Active Agents. Front. Robot. AI 3. doi:10.3389/frobt.2016.00004.

Thomas, V., Bengio, E., Fedus, W., Pondard, J., Beaudoin, P., Larochelle, H., et al. (2018). Disentangling the independently controllable factors of variation by interacting with the world. ArXiv Prepr. ArXiv180209484.

Thomas, V., Pondard, J., Bengio, E., Sarfati, M., Beaudoin, P., Meurs, M.-J., et al. (2017). Independently controllable factors. ArXiv Prepr. ArXiv170801289.

Toker, D., and Sommer, F. T. (2019). Information integration in large brain networks. PLOS Comput. Biol. 15, e1006807. doi:10.1371/journal.pcbi.1006807.

Tomasello, M. (2014). A Natural History of Human Thinking. Harvard University Press.

Tomasi, D., and Volkow, N. D. (2011). Association between Functional Connectivity Hubs and Brain Networks. Cereb. Cortex N. Y. NY 21, 2003-2013. doi:10.1093/cercor/bhq268.

Tononi, G., Boly, M., Massimini, M., and Koch, C. (2016). Integrated information theory: from consciousness to its physical substrate. Nat. Rev. Neurosci. 17, 450. doi:10.1038/nrn.2016.44.

Tononi, G., and Koch, C. (2015). Consciousness: here, there and everywhere? Philos. Trans. R. Soc. B Biol. Sci. 370, 20140167. doi:10.1098/rstb.2014.0167.

Toro-Serey, C., Tobyne, S. M., and McGuire, J. T. (2020). Spectral partitioning identifies individual heterogeneity in the functional network topography of ventral and anterior medial prefrontal cortex. NeuroImage 205, 116305. doi:10.1016/j.neuroimage.2019.116305.

Towlson, E. K., Vértes, P. E., Ahnert, S. E., Schafer, W. R., and Bullmore, E. T. (2013). The Rich Club of the C. elegans Neuronal Connectome. J. Neurosci. 33, 6380-6387. doi:10.1523/JNEUROSCI.3784-12.2013.

Traulsen, A., and Nowak, M. A. (2006). Evolution of cooperation by multilevel selection. Proc. Natl. Acad. Sci. U. S. A. 103, 10952-10955. doi:10.1073/pnas.0602530103.

Tversky, B. (2019). Mind in Motion: How Action Shapes Thought. 1 edition. New York: Basic Books.

Valyear, K. F., Philip, B. A., Cirstea, C. M., Chen, P.-W., Baune, N. A., Marchal, N., et al. (2020). Interhemispheric transfer of post-amputation cortical plasticity within the human somatosensory cortex. NeuroImage 206, 116291. doi:10.1016/j.neuroimage.2019.116291.

Varela, F. (1996). Neurophenomenology: A Methodological Remedy for the Hard Problem. J. Conscious. Stud. 3 , $330-49$.

Varela, F. J., Thompson, E. T., and Rosch, E. (1992). The Embodied Mind: Cognitive Science and Human Experience. Revised ed. edition. Cambridge, Mass.: The MIT Press.

Vasas, V., and Chittka, L. (2019). Insect-Inspired Sequential Inspection Strategy Enables an Artificial Network of Four Neurons to Estimate Numerosity. iScience 11, 85-92. doi:10.1016/j.isci.2018.12.009.

Vasileva, O., and Balyasnikova, N. (2019). (Re)Introducing Vygotsky's Thought: From Historical Overview to Contemporary Psychology. Front. Psychol. 10, 1515. doi:10.3389/fpsyg.2019.01515.

Vul, E., Goodman, N., Griffiths, T. L., and Tenenbaum, J. B. (2014). One and done? Optimal decisions from very few samples. Cogn. Sci. 38, 599-637. doi:10.1111/cogs.12101. 
Vygotsky, L. S. (1934). Thought and Language - Revised Edition. revised edition edition., ed. A. Kozulin Cambridge, Mass: The MIT Press.

Wan, Y., Wei, Z., Looger, L. L., Koyama, M., Druckmann, S., and Keller, P. J. (2019). Single-Cell Reconstruction of Emerging Population Activity in an Entire Developing Circuit. Cell 179, 355-372.e23. doi:10.1016/j.cell.2019.08.039.

Wang, J. X., Kurth-Nelson, Z., Kumaran, D., Tirumala, D., Soyer, H., Leibo, J. Z., et al. (2018). Prefrontal cortex as a meta-reinforcement learning system. Nat. Neurosci. 21, 860. doi:10.1038/s41593-018-0147-8.

Wang, T., and Roychowdhury, J. (2019). OIM: Oscillator-based Ising Machines for Solving Combinatorial Optimisation Problems. in International Conference on Unconventional Computation and Natural Computation (Springer), 232-256.

Wens, V., Bourguignon, M., Vander Ghinst, M., Mary, A., Marty, B., Coquelet, N., et al. (2019). Synchrony, metastability, dynamic integration, and competition in the spontaneous functional connectivity of the human brain. NeuroImage. doi:10.1016/j.neuroimage.2019.05.081.

Whitehead, K., Meek, J., and Fabrizi, L. (2018). Developmental trajectory of movement-related cortical oscillations during active sleep in a cross-sectional cohort of pre-term and full-term human infants. Sci. Rep. 8, 1-8. doi:10.1038/s41598-018-35850-1.

Wicken, M., Keogh, R., and Pearson, J. (2019). The critical role of mental imagery in human emotion: insights from Aphantasia. bioRxiv, 726844. doi:10.1101/726844.

Wijesinghe, R., Protti, D. A., and Camp, A. J. (2015). Vestibular Interactions in the Thalamus. Front. Neural Circuits 9. doi:10.3389/fncir.2015.00079.

Willms, A. R., Kitanov, P. M., and Langford, W. F. Huygens' clocks revisited. R. Soc. Open Sci. 4, 170777. doi:10.1098/rsos.170777.

Wittmann, M. (2017). Felt Time: The Science of How We Experience Time. Reprint edition. Cambridge, MA: The MIT Press.

Zajchowski, C. A. B., Schwab, K. A., and Dustin, D. L. (2017). The experiencing self and the remembering self: Implications for leisure science. Leis. Sci. 39, 561-568. doi:10.1080/01490400.2016.1209140.

Zeman, A., Dewar, M., and Della Sala, S. (2015). Lives without imagery - Congenital aphantasia. Cortex 73, 378380. doi:10.1016/j.cortex.2015.05.019.

Zhang, H., Watrous, A. J., Patel, A., and Jacobs, J. (2018). Theta and Alpha Oscillations Are Traveling Waves in the Human Neocortex. Neuron 98, 1269-1281.e4. doi:10.1016/j.neuron.2018.05.019. 